Received 11th May 2020

Accepted 6th July 2020

DOI: 10.1039/d0qo00567c

rsc.li/frontiers-organic

\title{
Promising reagents for difluoroalkylation
}

Cite this: Org. Chem. Front., 2020, 7, 2538

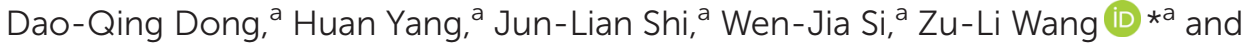 \\ Xin-Ming $X u^{b}$
}

This review describes recent advances in difluoroalkylation reactions using different substrates. Generally speaking, $\mathrm{RCF}_{2}$ radical is generally proposed in the mechanism of these reactions. At present, the most used difluoroalkylation reagent is $\mathrm{RCF}_{2} \mathrm{X}$, which can be reduced by metal, photocatalyst or bases to $\mathrm{RCF}_{2}$ radical. Various substrates such as alkenes, alkynes, amines and so on could well be applied to these difluoroalkylation reactions.

\section{Introduction}

Because of their special physicochemical properties, difluorinated compounds have an important role in agrochemicals, pharmaceuticals, and materials sciences. ${ }^{1-3}$ Therefore, the development of efficient and simple methods for the introduction of difluoroalkyl groups has received more and more attention in recent years. ${ }^{4-8}$ At present, various reagents such as $\mathrm{HCF}_{2} \mathrm{Cl}_{1}{ }^{9} \quad \mathrm{BrCF}_{2} \mathrm{P}(\mathrm{O})(\mathrm{OEt})_{2},{ }^{10} \quad \mathrm{TMSCF}_{2} \mathrm{Br}^{11,12} \mathrm{HFPO},{ }^{13}$ $\mathrm{CF}_{3} \mathrm{ZnBr}_{2} \cdot \mathrm{CH}_{3} \mathrm{CN},{ }^{5} \mathrm{FSO}_{2} \mathrm{CF}_{2} \mathrm{CO}_{2} \mathrm{H}^{14}$ and so on ${ }^{4,15-18}$ are used for difluoroalkylation reactions. But some side reactions such as dimerization, protonation and other unknown byproducts accompany the difluoroalkylation reaction when some of these reagents especially metal species are employed. As early as 2001, the Chen group realized the fluoroalkylation of aromatic compounds with per(poly)fluoroalkyl chlorides mediated by sodium dithionite. The single-electron transfer (SET) mechanism was proposed for the fluoroalkylation reaction. ${ }^{18 b}$ Difluoroalkyl halides as a class of efficient and readily available reagents for difluoroalkylation reactions have become the most widely used in organic synthesis in recent years. In 2018, the Zhang group summarized transition-metal-catalyzed difluoroalkylation with difluoroalkyl halides via cross-coupling. ${ }^{4}$ According to the pathways by which the difluoroalkyl halides react, they classified these reactions into four categories: nucleophilic difluoroalkylation, electrophilic difluoroalkylation, radical difluoroalkylation and difluoromethylation by metal-difluorocarbene coupling. There have been some other reviews about difluoroalkylation $\left(\mathrm{CF}_{2} \mathrm{R}\right)$ reactions, ${ }^{4,6,19-22}$ and these reactions will not be described in this review. Besides metal-catalyzed difluoroalkylation reactions, difluor-

\footnotetext{
${ }^{a}$ College of Chemistry and Pharmaceutical Sciences, Qingdao Agricultural University, Qingdao 266109, China. E-mail: wangzulichem@163.com

${ }^{b}$ College of Chemistry and Chemical Engineering, Yantai University, Yantai 264005,
} China oalkylation reactions which are conducted under metal-free conditions also have made much progress in recent years, and these reactions will be described in this review. This review mainly describes the difluoroalkylation reactions reported in the last three years. According to the different substrates, we classify these difluoroalkylation reactions into eight types.

2. Difluoroalkylation with alkenes

2.1. Carbodifluoroalkylation with alkenes

2.2. Hydrodifluoroalkylation with alkenes

2.3. Heterodifluoroalkylation with alkenes

2.4. Other difluoroalkylation with alkenes

3. Difluoroalkylation with alkynes

3.1. Carbodifluoroalkylation with alkynes

3.2. Hydrodifluoroalkylation with alkynes

3.3. Heterodifluoroalkylation with alkynes

3.4. Other difluoroalkylation with alkynes

4. Difluoroalkylation with arene

5. Difluoroalkylation with alkyl C-H bond

6. Difluoroalkylation with isocyano

7. Difluoroalkylation with amine

8. Difluoroalkylation with $\mathrm{C}=\mathrm{N}$

9. Difluoroalkylation with $\mathrm{C}=\mathrm{O}$

\section{Difluoroalkylation with alkenes}

\subsection{Carbodifluoroalkylation with alkenes}

In the past few years, a significant renaissance of photocatalysis in the field of organic chemistry has been $\operatorname{seen}^{7,23-33}$ since the pioneer works reported by MacMillan ${ }^{34}$ and Yoon $^{35}$ in 2008. With the assistance of visible light, $\mathrm{RCF}_{2} \mathrm{X}$ could serve as an important synthon for difluoroalkylation reactions in organic chemistry. The reduction of $\mathrm{RCF}_{2} \mathrm{X}$ in the presence of a photocatalyst was one of the most common strategies for the formation of $\mathrm{RCF}_{2}$ radical. Because of its high reactivity, $\mathrm{RCF}_{2}$ 


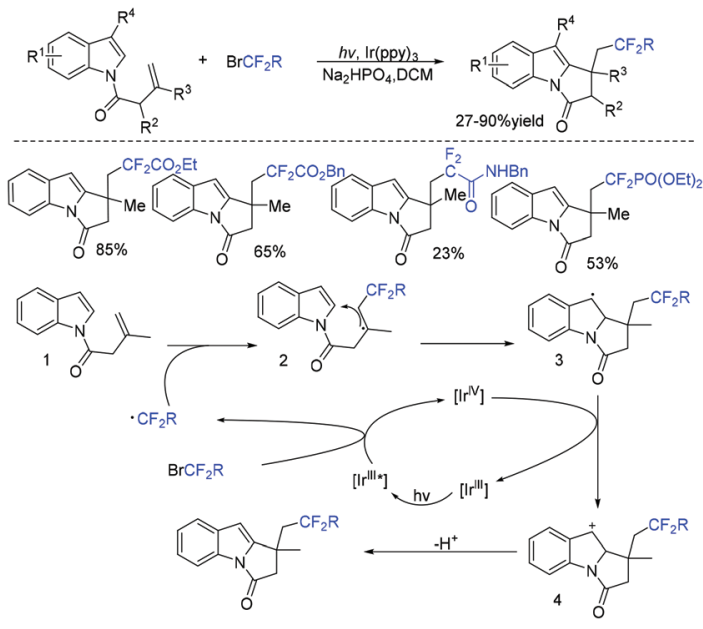

Scheme 1 The difluoroalkylation/cyclization reaction of $\mathrm{N}$-(but-2enoyl)indoles.

radical could react with various substrates to construct complicated molecules via difuloroalkylation reactions. ${ }^{36-41}$

In 2018, the $\mathrm{Li}$ group demonstrated that difluoroalkylated pyrrolo[1,2- $\alpha$ indoles with a quaternary carbon center can be efficiently synthesized via difluoroalkylation and cyclization cascade reaction of $\mathrm{N}$-(but-2-enoyl)indoles with $\mathrm{BrCF}_{2} \mathrm{R}$ (Scheme 1). ${ }^{42}$ In the presence of visible light, products with good to high yields were generated. A plausible reaction mechanism is outlined in Scheme 1. Initially, $\mathrm{RCF}_{2}$ radical was generated from the oxidation of excited photocatalyst $\left[\mathrm{Ir}^{* 3^{3+}}\right]$ with $\mathrm{RCF}_{2} \mathrm{Br}$. The addition of $\mathrm{RCF}_{2}$ radical to 1 produced intermediate 2. Subsequently, intermediate $\mathbf{3}$ was formed from intramolecular radical cyclization of intermediate B on $\mathrm{C} 2$ of the indole ring. Then oxidation of intermediate 3 by the $\left[\operatorname{Ir}^{4+}\right]$ complex occurred to deliver intermediate 4 and the $\left[\mathrm{Ir}^{3+}\right]$ catalyst. Finally, deprotonation of this cationic intermediate 4 by base afforded the final product.

This visible light induced radical addition/cyclization strategy could also be applied for the reaction of biaryl vinyl ethers with $\mathrm{BrCF}_{2} \mathrm{CO}_{2} \mathrm{Et}$ (Scheme 2). ${ }^{43}$ 6-Fluoroalkylchromenes with moderate to good yields were obtained. Control experiments indicated that $\mathrm{RCF}_{2}$ radical might be involved in the mechanism, and a radical addition to the carbon-carbon double bond might occur prior to the cyclization process.

The copper-mediated difluoromethylenation of $N$-arylacrylamides with benzo-1,3-oxazolic difluoromethyl-

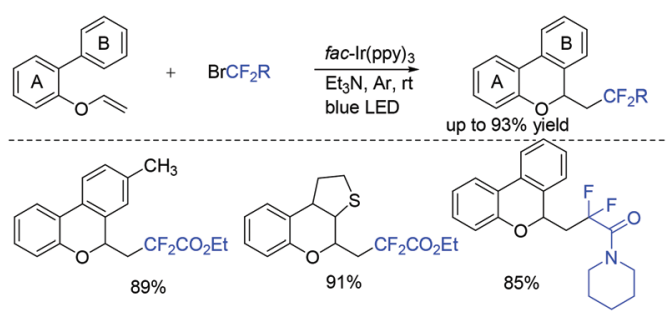

Scheme 2 The radical addition/cyclization of biaryl vinyl ethers.

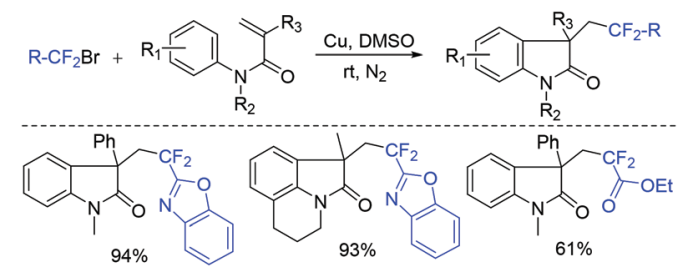

Scheme 3 The $\mathrm{N}$-arylacrylamides.

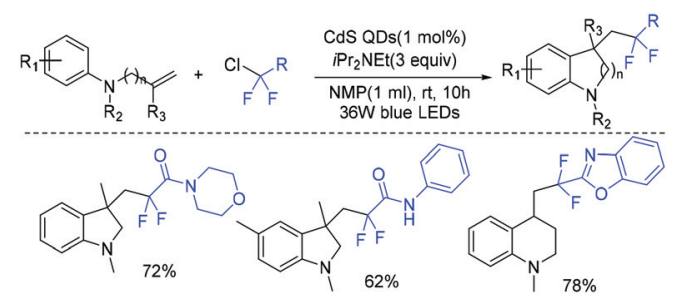

Scheme 4 The cadmium sulfide quantum-dot-photocatalyzed cascade cyclization of unactivated olefins.

bromide was described by the Jiang group (Scheme 3) ${ }^{44} \mathrm{RCF}_{2}$ radical, which was generated from $\mathrm{Cu}(0)$-mediated SET with $\mathrm{RCF}_{2} \mathrm{Br}$, could add to the terminal $\mathrm{C}=\mathrm{C}$ double bond of $\mathrm{N}$-arylacrylamide and then underwent intramolecular radical cyclization to form the desired product.

In 2019, Feng and coworkers reported the cadmium sulfide quantum-dot-photocatalyzed cascade cyclization of ethyl chlorodifluoroacetate with unactivated olefins (Scheme 4$)^{45}$ Control experiments showed that a radical intermediate was involved in this reaction. Visible-light-absorbing CdS QDs which could transform difluoromethyl chlorides to difluoromethyl radical played an important role in this strategy.

The Yang group found that $\mathrm{CF}_{2}$-containing benzoxepine derivatives can be synthesized from visible-light-induced external radical-triggered annulation. ${ }^{46}$ In addition, different products were obtained if the substituents on the nitrogen were different. A plausible mechanism is outlined in Scheme 5 . Initially, the excited state $\left[\operatorname{Ir}(\mathrm{dtbbpy})(\mathrm{bpy})_{2} \mathrm{PF}_{6}\right]^{*}$ which was generated under visible-light irradiation was oxidized by $\mathrm{BrCF}_{2} \mathrm{CO}_{2} \mathrm{Et}$ to generate $\left[\mathrm{Ir}(\mathrm{Iv})(\mathrm{dtbbpy})(\mathrm{bpy})_{2} \mathrm{PF}_{6}\right]^{+}$complex and $\mathrm{RCF}_{2}$ radical species 5. Subsequently, radical intermediate 6 was formed via sequential radical addition between $\mathrm{RCF}_{2}$ radical and the propenyl group of the substrate. Then intermediate 6 was oxidized by $\left[\operatorname{Ir}(\mathrm{rv})(\mathrm{dtbbpy})(\mathrm{bpy})_{2} \mathrm{PF}_{6}\right]^{+}$to form iminium intermediate 7 with the concurrent regeneration of $\left[\operatorname{Ir}(\mathrm{dtbbpy})(\mathrm{bpy})_{2} \mathrm{PF}_{6}\right]$. Finally, the corresponding product was generated from the hydrolyzation of iminium ion $\mathrm{C}$ by water (path a). Additionally, when nitrogen was monosubstituted iminium ion, the corresponding enamine was formed via the deprotonation and tautomerization reaction (path b).

The Sun group found $\mathrm{N}$-arylacrylamides could also react with $\mathrm{BrCF}_{2} \mathrm{CO}_{2} \mathrm{R}$ smoothly for assembly of difluoroalkylphenanthridine derivatives induced by visible light (Scheme 6). ${ }^{47}$ 

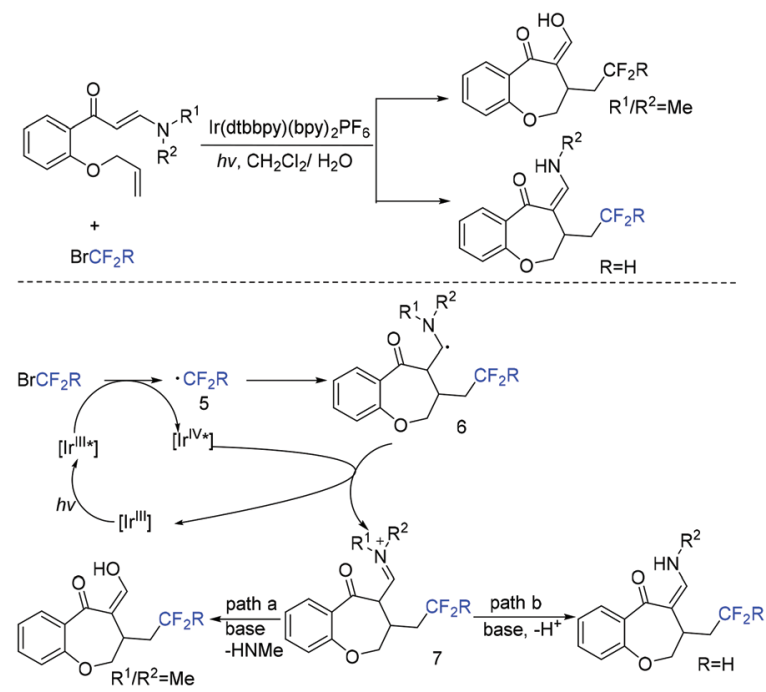

Scheme 5 The synthesis of $\mathrm{CF}_{2}$-containing benzoxepine derivatives.

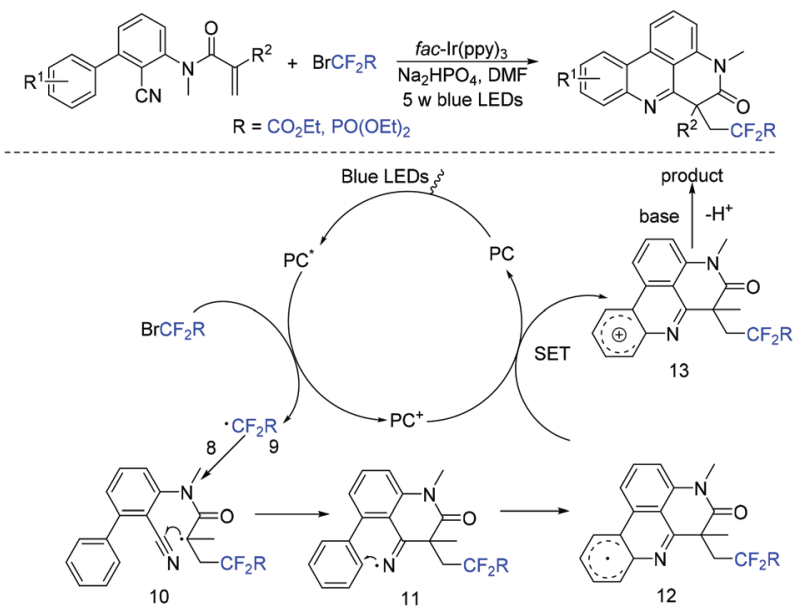

Scheme 6 The assembly of difluoroalkylphenanthridine derivatives.

Besides, fluoroalkylating reagents $\mathrm{CF}_{3} \mathrm{SO}_{2} \mathrm{Cl}$ and $\mathrm{BrCF}_{2} \mathrm{PO}$ $(\mathrm{OEt})_{2}$ were also well applicable to this reaction. Fluoroalkyl radical which was generated from oxidative quenching of the excited state $\mathrm{PC}^{*}$ by the fluorine reagents was proposed in the mechanism. The subsequent intermolecular radical addition to the $\mathrm{C}=\mathrm{C}$ double bond of $\mathrm{N}$-arylacrylamides 8 afforded the radical intermediate $\mathbf{1 0}$, followed by intramolecular radical addition to the cyano group affording iminyl radical 11 . Subsequently the homolytic aromatic substitution process, oxidation and deprotonation reaction occurred in sequence to afford the final products.

In 2018, the Shi group developed a facile method to construct diverse difluorinated quinoline-2,4-diones via $\mathrm{Cu}$-catalyzed direct difluoromethylation of activated alkenes through a difluoromethyl radical addition/cyclization reaction (Scheme 7). It is noteworthy that visible-light photoredox catalysis was also effective for this reaction. ${ }^{48}$

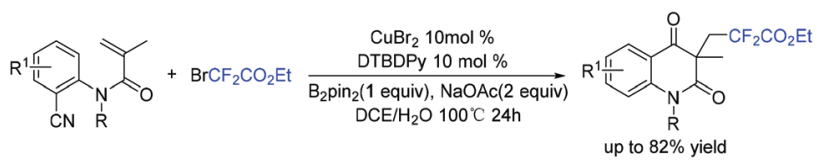

Scheme 7 The synthesis of difluorinated quinoline-2,4-diones catalyzed by copper.

Chroman-4-ones are an important motif in medicinal compounds and biologically active molecules. ${ }^{4-51}$ In 2019, the Zhou group described a visible-light photoredox-catalyzed radical cascade cyclization of alkenyl aldehydes with $\mathrm{BrCF}_{2} \mathrm{COR}$ for the synthesis of various difluoroacetylated chroman-4-ones (Scheme 8). ${ }^{52}$ The addition reaction of fluoroalkyl radical 15 to 16 afforded the intermediate 17, which then underwent intramolecular cyclization to give the intermediate 18. Subsequently, 1,2-H shift of E resulted in intermediate 19. Then, intermediate 19 was oxidized by Ir(Iv) to afford carbocation 20, which was converted to the desired product by deprotonation.

Difluoroacetic acids were also found to be good difluoroalkyl radical precursors for decarboxylative difluoroalkylation. ${ }^{53-57}$ In 2019, the Zhu group realized visible-light-induced acyldifluoroalkylation of alkenyl aldehydes with difluoroacetic acids (Scheme 9). ${ }^{58} \mathrm{PhI}(\mathrm{OAc})_{2}$ should be added as the oxidant, which could react with the excited state $\operatorname{Ir}($ III)* and difluoroacetic acids via SET process to give difluoromethyl radical.

The group of Tu and Jiang found that fluoroalkylated (Z)-1indenones could be efficiently synthesized via hydrofluoroalkylation of 1,6-enynes with ethyl bromodifluoroacetate in the presence of photocatalysts. ${ }^{59}$ THF behaved as a hydrogen source in this transformation. The proposed mechanism is shown in Scheme 10. Oxidation of Ir*(III) by fluoroalkyl bromides resulted in fluoroalkyl radical 21. The addition of radical 21 to 1,6-enynes generated radical 22 , which was followed by cyclization to produce vinyl radical 23 . Finally, radical

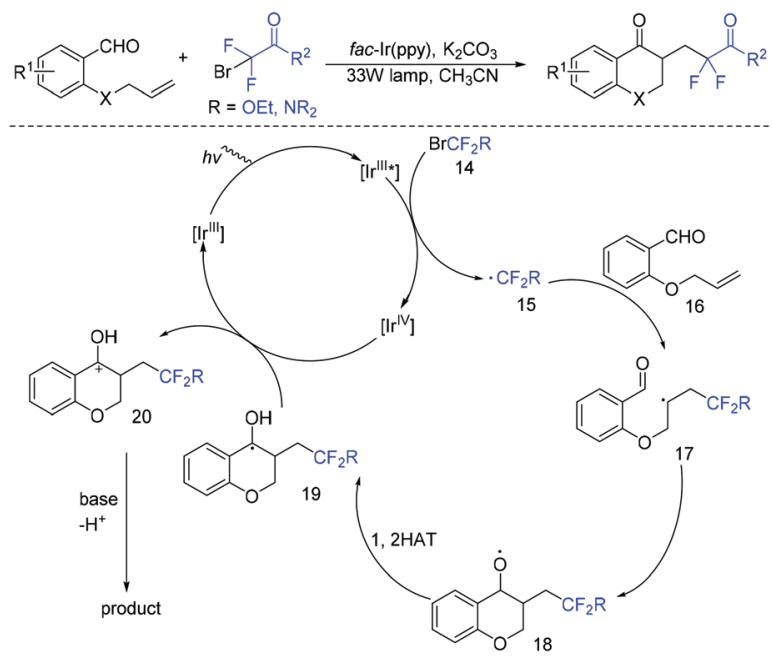

Scheme 8 The cyclization of alkenyl aldehydes with $\mathrm{BrCF}_{2} \mathrm{COR}$. 

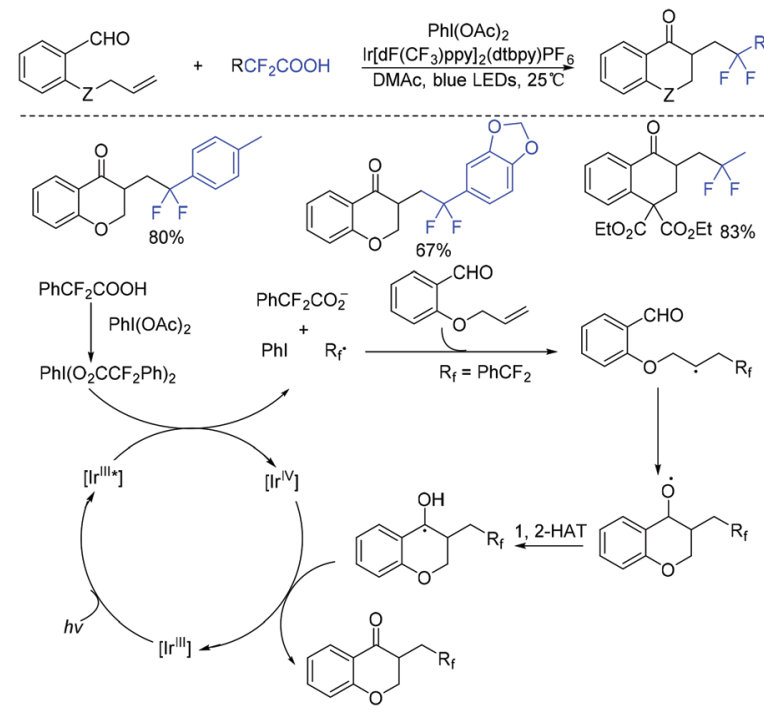

Scheme 9 The acyldifluoroalkylation of alkenyl aldehydes with difluoroacetic acids.

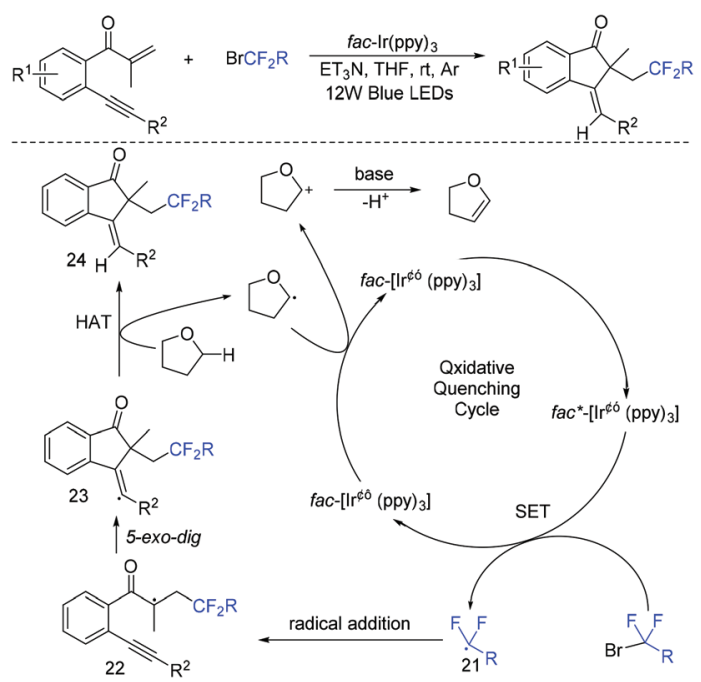

Scheme 10 The synthesis of fluoroalkylated (Z)-1-indenones.

23 abstracted hydrogen from THF to provide the desired product. In addition, fluoroalkyl and halo groups were all incorporated into 1-indenone skeleton when copper catalysis was used instead of photocatalysis.

The Liang group found that 1,6-enynes were good substrates for difluoroalkylation with $\mathrm{ICF}_{2} \mathrm{CO}_{2}$ Et (Scheme 11). ${ }^{60}$ In the absence of metal catalysts, two different difluoroalkylated cyclization products with various functional groups and high stereoselectivity were obtained under the condition that only base was used. The properties of the bases were key factors to give different difunctionalized cyclization products. The reaction of base with $\mathrm{ICF}_{2} \mathrm{CO}_{2} \mathrm{Et}$ to afford $\mathrm{CF}_{2} \mathrm{CO}_{2} \mathrm{Et}$ radical via EDA complex was suggested in the catalytic cycle.

The palladium-catalyzed three-component cascade difluoroalkylation and arylation of 1,6-enynes with ethyl difluoro-

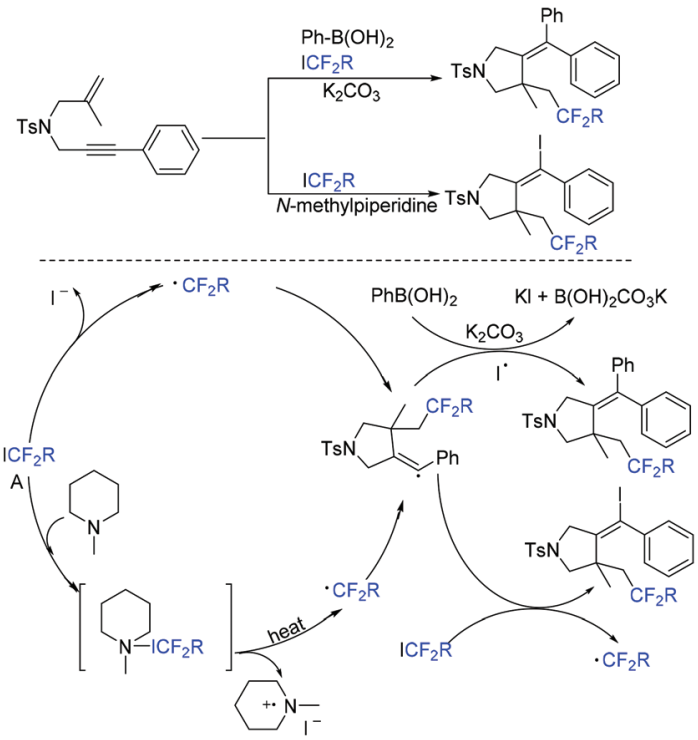

Scheme 11 The difluoroalkylation of 1,6-enynes with $\mathrm{ICF}_{2} \mathrm{CO}_{2} \mathrm{Et}$.

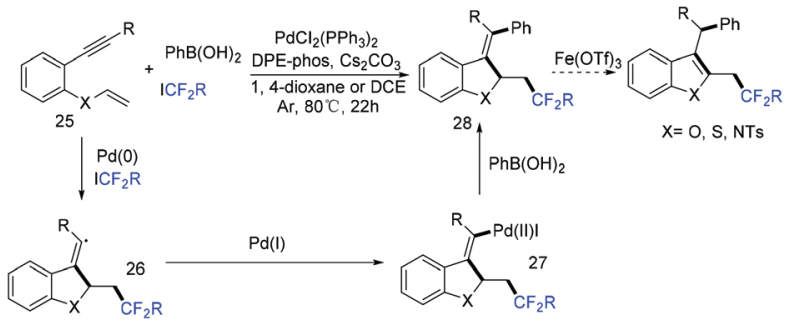

Scheme 12 The synthesis of fluoroalkylated (Z)-1-indenones.

iodoacetate and phenylboronic acid was realized by the Zhang group (Scheme 12). ${ }^{61}$ The products can be converted into $\mathrm{CF}_{2}{ }^{-}$ containing benzofurans, benzothiophenes and indoles through an $\mathrm{Fe}(\mathrm{OTf})_{3}$-catalyzed isomerization. Reduction of $\mathrm{ICF}_{2} \mathrm{CO}_{2}$ Et with $\operatorname{Pd}(0)$ to form $\mathrm{CF}_{2} \mathrm{CO}_{2}$ Et radical, transmetalation process between the intermediate 27 and phenylboronic acid process were proposed in the reaction mechanism.

Difluoroacyl heterocyclic compounds have been found in various biologically active compounds and agrochemicals. ${ }^{62,63}$ Much effort has been devoted to these compounds. ${ }^{64-68}$ In 2019, the copper-mediated radical cyclization of naphthalenyl iododifluoromethyl ketones with olefins was demonstrated by the $\mathrm{Wu}$ group (Scheme 13), ${ }^{69}$ providing difluoroacyl compounds with moderate yields. Mechanistic investigations implied that difluorinated radical intermediate which was generated from the reduction of $\mathrm{RCF}_{2} \mathrm{I}$ by $\mathrm{Cu}(0)$ was involved in the reaction pathway.

In 2019, the Wang group described the copper $/ \mathrm{B}_{2}$ pin $_{2}$-catalyzed difluoroalkylation of methylenecyclopropanes with bromodifluorinated acetates and acetamides (Scheme 14). ${ }^{70}$ Various substrates reacted well in this system. Both the catalyst and an inert atmosphere are necessary for the success of this reaction. A tandem radical process involving ring-opening/ intramolecular cyclization was proposed in the catalytic cycle. 


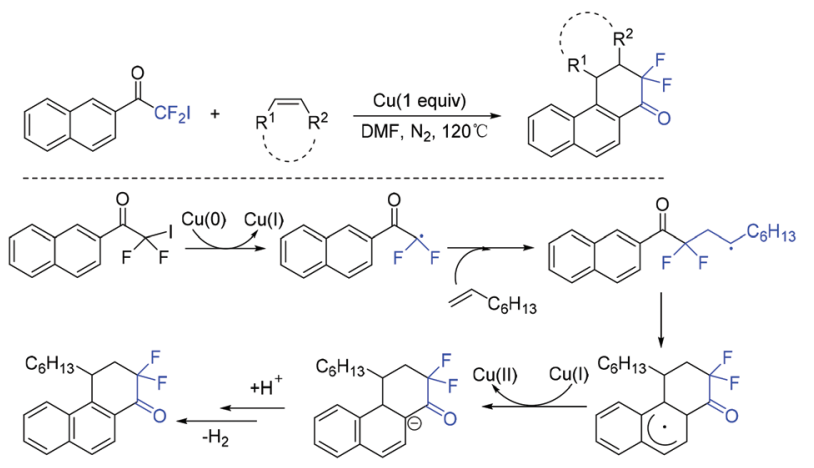

Scheme 13 The copper-mediated radical cyclization of naphthalenyl iododifluoromethyl ketones.

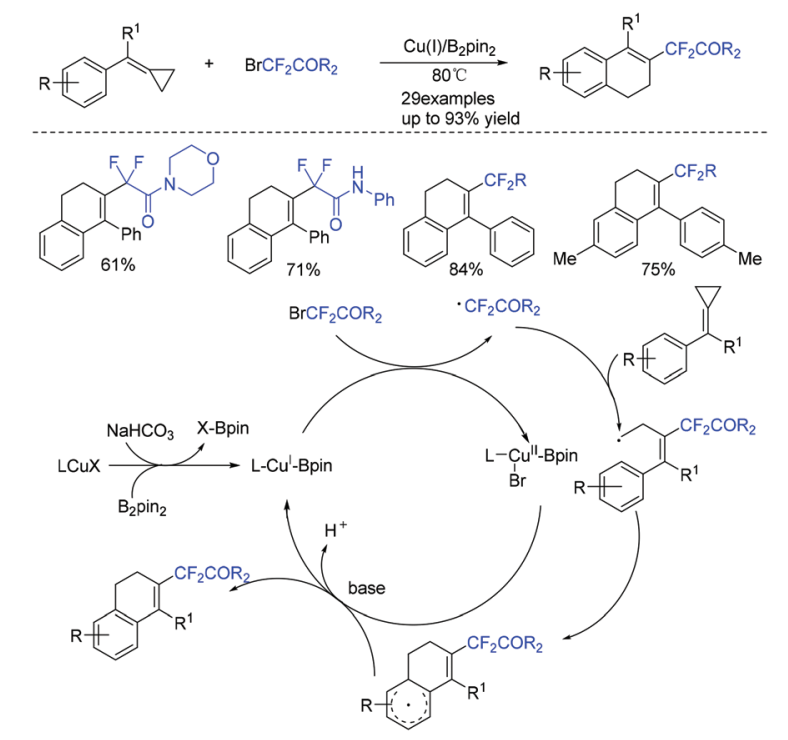

Scheme 14 The copper/B ${ }_{2}$ pin $_{2}$-catalyzed difluoroalkylation methylenecyclopropanes.

The first difunctionalization of unactivated alkenes through desulfonylation-initiated distal alkenyl migration was reported by the Wang group. Previously unknown 3,3-difluoro-5-styrylpiperidin-2-one derivatives bearing a quaternary stereocenter can be efficiently synthesized using this method (Scheme 15). ${ }^{71} \mathrm{~A}$ radical mechanism was suggested for this transformation. Initially, fluoroalkyl radical 29 and $\mathrm{Cu}$ (II) species are generated from the oxidation of $\mathrm{Cu}(\mathrm{I})$ species by $\mathrm{BrCF}_{2} \mathrm{R}_{3}$ through a SET process. Then fluoroalkyl radical 29 selectively attacks the less sterically hindered terminal alkene to give the transient alkyl radical 30. The addition reaction of the $\mathrm{RCF}_{2}$ radical 30 to the internal double bond occurred to generate the cyclized radical 31. Radical 31 will undergo rapid desulfonylation to form the key $\mathrm{N}$-centered radical 32. Finally, an annulation proceeded rapidly and delivered the products 3,3-difluoro-5-styrylpiperidin-2-ones.

A novel photocatalytic 1,2-heterocycle migration reaction of allylic alcohols with $\mathrm{BrCF}_{2} \mathrm{CO}_{2} \mathrm{Et}$ was described by the Noel

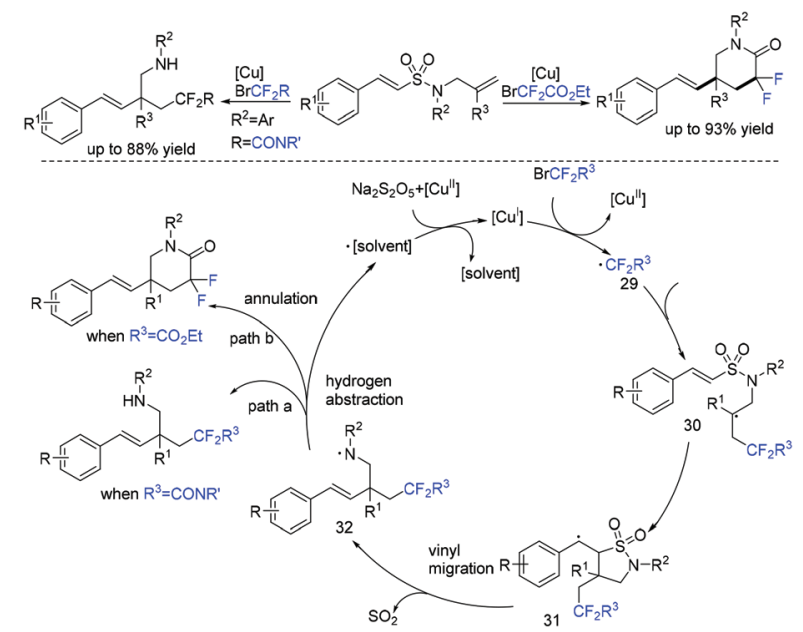

Scheme 15 The difunctionalization of unactivated alkenes through desulfonylation.

group, affording various $\beta$-difluorinated $\alpha$-aryl heterocyclic ketones (Scheme 16). ${ }^{72}$ The application of continuous flow allowed shorter reaction times, higher selectivity and opportunities to scale the chemistry. The addition of $\mathrm{CF}_{2} \mathrm{CO}_{2} \mathrm{Et}$ radical generated from the oxidation of $f a c-\left[\operatorname{Ir}(\mathrm{ppy})_{3}\right]^{*}$ to the olefin generated intermediate 33. Then 1,2-heterocycle migration of 33 resulted in 35, which was further converted to the desired product via oxidation. The $\mathrm{Cu}_{2} \mathrm{O}$-catalyzed phosphonyldifluoromethylation and ethoxycarbonyldifluomethylation of allylic alcohols through a radical 1,2-aryl migration was also realized by the Xiong group (Scheme 17). ${ }^{73}$

Without the need of photocatalyst, a light-mediated TruceSmiles arylative rearrangement mediated by an organic base

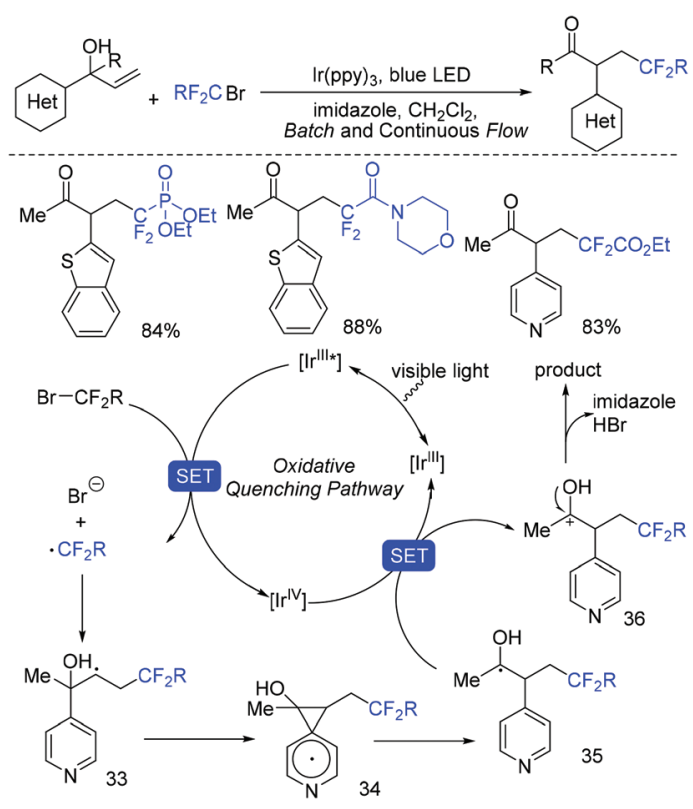

Scheme 16 The photocatalytic difluoromethylation of allylic alcohols. 


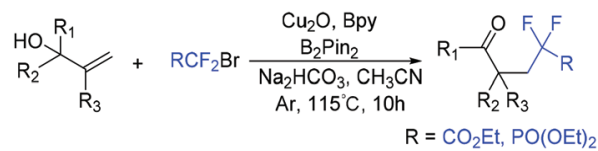

Scheme 17 The $\mathrm{Cu}_{2} \mathrm{O}$-catalyzed difluoromethylation of allylic alcohols.

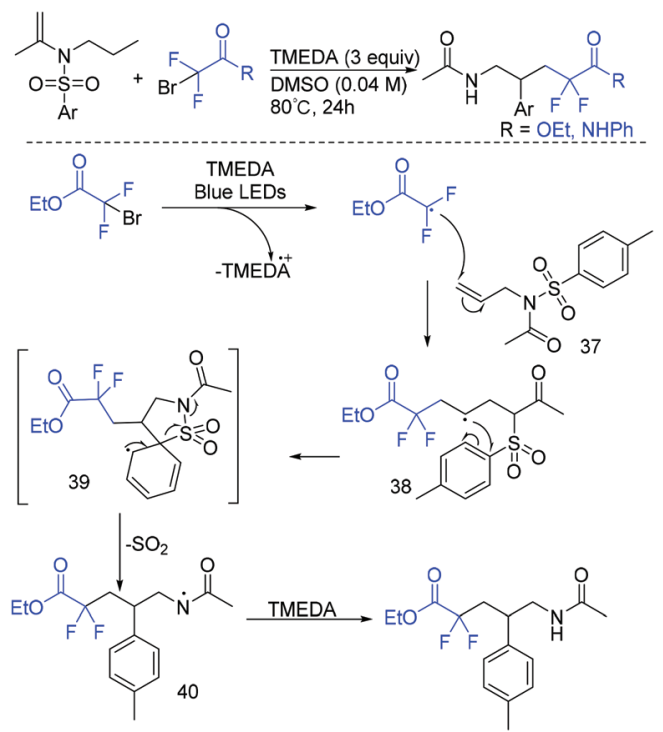

Scheme 18 The light-mediated arylative rearrangement.

was described by the group of Duong and Greaney (Scheme 18). ${ }^{74}$ This transformation simultaneously introduced an aryl ring and a difluoroacetate moiety across unactivated alkenes. A radical mechanism was proposed for this reaction. Different from previous reports, $\mathrm{CF}_{2} \mathrm{CO}_{2} \mathrm{Et}$ radical could be generated from the reduction of $\mathrm{BrCF}_{2} \mathrm{CO}_{2} \mathrm{Et}$ by TEMDA induced by visible light. The addition reaction of $\mathrm{CF}_{2} \mathrm{CO}_{2} \mathrm{Et}$ radical to alkenes 37 resulted in radical 38. Then, TruceSmiles aryl shift with extrusion of $\mathrm{SO}_{2}$ and hydrogen atom transfer occurred in sequence to afford the desired product.

The visible-light-mediated tandem radical difluoroalkylation and alkynylation of unactivated alkenes with $\mathrm{BrCF}_{2} \mathrm{COR}$ was realized by the Zhu group (Scheme 19). ${ }^{75}$ However, an aliphatic alkynyl substrate was not suitable for this transformation because of the rearrangement of the starting material and undesired decomposition. The $\mathrm{C}-\mathrm{C}$ bond activation via intramolecular 1,4-alkynyl migration was involved in the mechanism.

Another organic base-promoted difluoroalkylation reaction of 1,4-enynes with $\mathrm{ICF}_{2} \mathrm{CO}_{2}$ Et was realized by yje Liang group. ${ }^{76}$ Various functional groups were compatible with this reaction. A possible mechanism is outlined in Scheme 20. The direct interaction of $N$-methylpiperidine with $\mathrm{ICF}_{2} \mathrm{CO}_{2} \mathrm{Et}$ was proposed to form $\mathrm{CF}_{2} \mathrm{CO}_{2} \mathrm{Et}$ radical. Alkynyl radical migration of $\mathbf{4 1}$ was also proposed in the mechanism.

The first intermolecular alkynyl-difluoroalkylation of unactivated alkenes via visible-light-induced three-component reac-
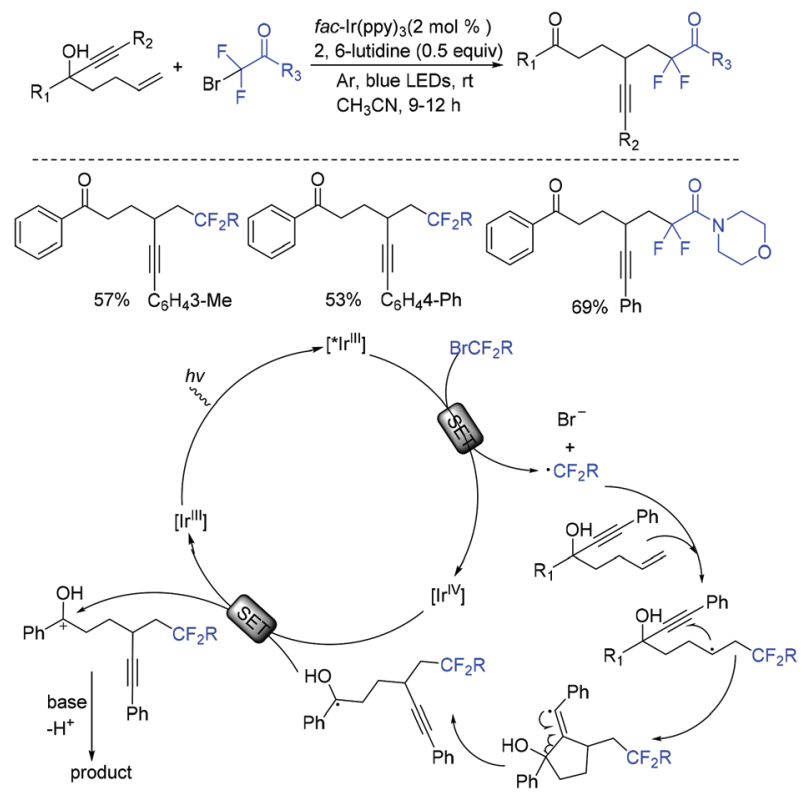

Scheme 19 The light-mediated difluoroalkylation and alkynylation of unactivated alkenes.

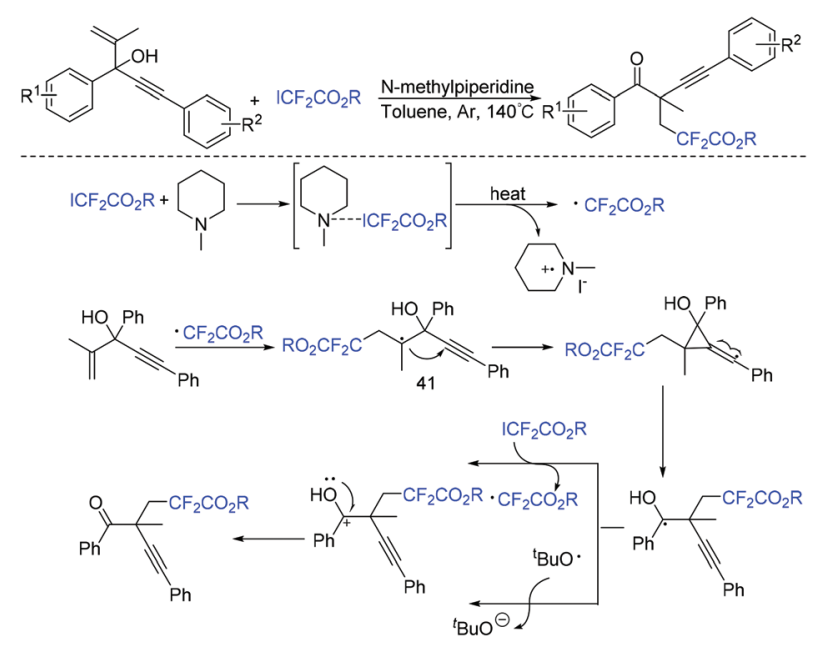

Scheme 20 The base-promoted difluoroalkylation reaction of 1,4enynes with $\mathrm{ICF}_{2} \mathrm{CO}_{2} \mathrm{Et}$.

tion of difluoroalkyl halides, unactivated alkenes and alkynyl sulfones was realized by the Zhu group (Scheme 21). ${ }^{77}$ This reaction provides a simple and efficient route to the construction of synthetically valuable $\beta$-difluoroalkylated alkynes. The reaction of Ir $^{* \text { III }}$ and $\mathrm{R}_{\mathrm{f}} \mathrm{X}$ occurred to form difluoroalkyl radical, which was followed by addition to alkenes to afford intermediate II. Then the addition of intermediate II to alkynyl sulfones and $\beta$-elimination occurred in sequence to produce the final product. It is worthwhile to note that $\mathrm{Et}_{3} \mathrm{~N}$ plays multiple roles such as a base and a reductant in this reaction. Additionally, $\operatorname{Ir}(\mathrm{II}) / \operatorname{Ir}(\mathrm{III})$ pathway was also possible for this transformation. 


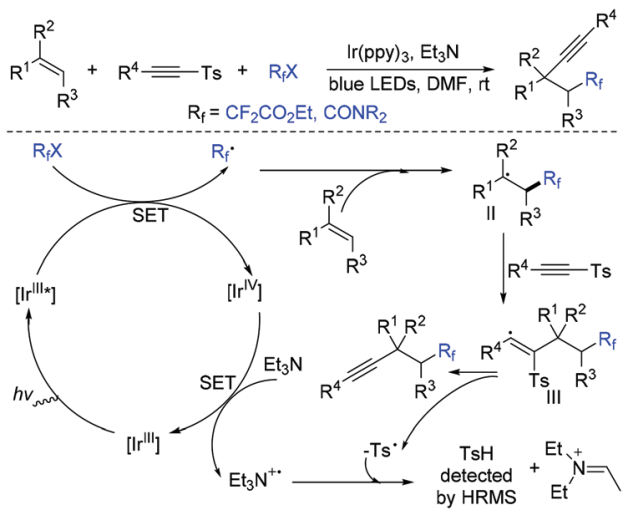

Scheme 21 The alkynyl-difluoroalkylation of unactivated alkenes.

As is well known, allenes are highly reactive substrates and play an important role in organic chemistry. ${ }^{78,79}$ A nickel-catalyzed 1,4-carbofluoroalkylation of 1,3-enynes with boronic acids and fluoroalkyl halides to access structurally diverse fluoroalkylated allenes was reported by the Wang group (Scheme 22). ${ }^{80}$ The use of nickel to generate fluoroalkyl radicals from $\mathrm{BrCF}_{2} \mathrm{CO}_{2} \mathrm{Et}$ (rate-determining step) and reductive elimination were proposed for this reaction.

Besides 1,3-enynes, alkenes are also good substrates to react with boronic acids and fluoroalkyl halides, producing $\gamma$-arylation of carbonyl compounds. The Shu group found that the employment of copper and visible-light catalysis was essential for this reaction. ${ }^{81}$ The mechanism is shown in Scheme 23. The reduction of $\mathrm{BrCF}_{2} \mathrm{CO}_{2}$ Et with excited $\operatorname{Ir}^{*}(\mathrm{III})$ photocatalyst would give radical intermediate 42 and the oxidized photocatalyst $\operatorname{Ir}(\mathrm{Iv}) . \mathbf{4 2}$ would be trapped by alkenes to
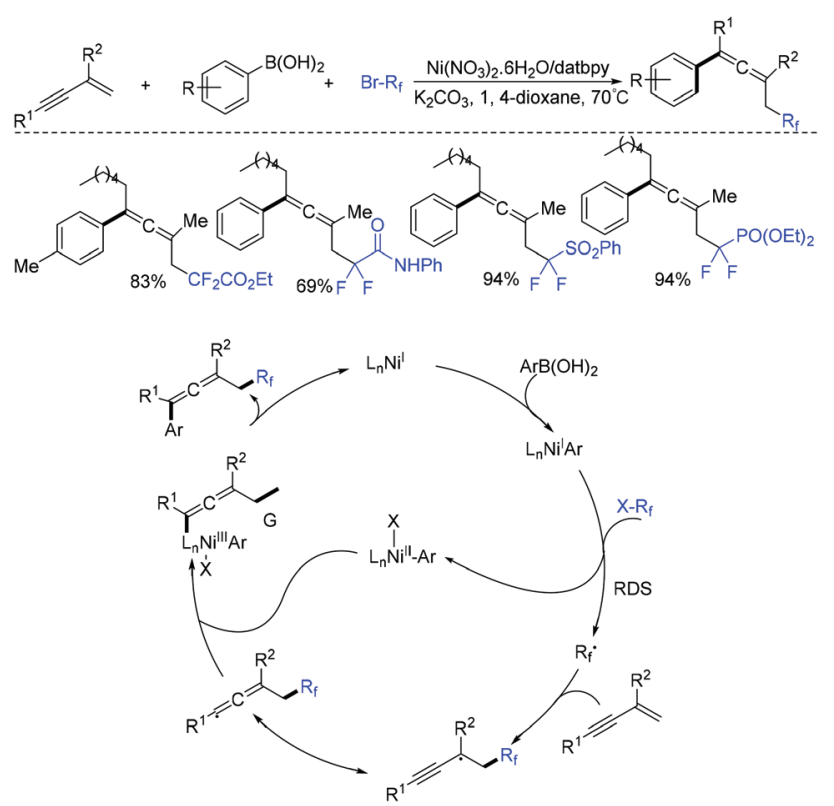

Scheme 22 The nickel-catalyzed 1,4-carbofluoroalkylation of 1,3enynes.

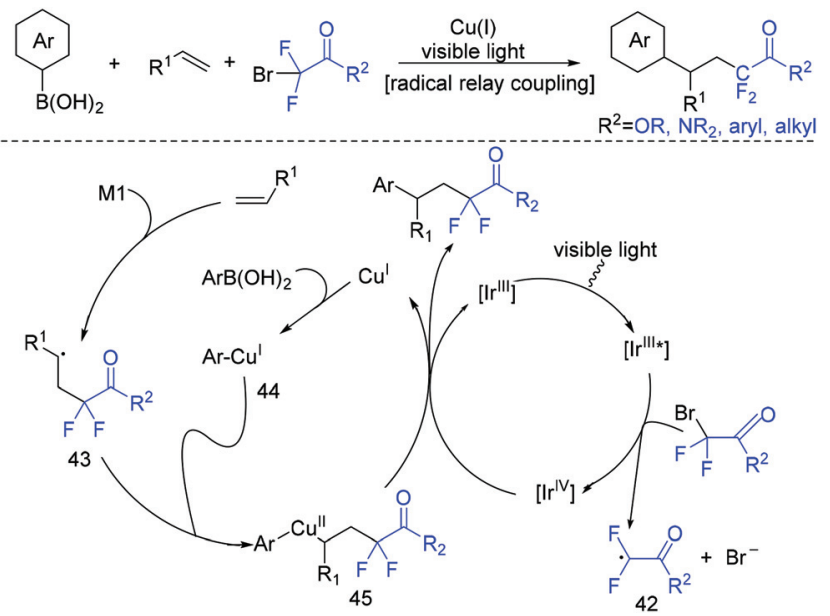

Scheme 23 The process of $\gamma$-arylation of carbonyl compounds.

generate a new alkyl radical intermediate 43. In the presence of a base, the arylcopper intermediate $\mathbf{4 4}$ was delivered from the reaction of $\mathrm{Cu}(\mathrm{I})$ with aryl boronic acid, which was followed by recombination with $\mathbf{4 3}$ to give $\mathbf{4 5}$. Then, $\mathbf{4 5}$ would undergo reductive elimination to give the final product with the concurrent regeneration of $\operatorname{Ir}(\mathrm{III})$ and $\mathrm{Cu}(\mathrm{I})$ species.

In 2019, the perfluoroalkylative pyridylation of alkenes via 4-cyanopyridineboryl radicals was developed by the Li group (Scheme 24). ${ }^{82}$ Density functional theory calculations and experimental studies suggested that the 4-cyanopyridineboryl radicals generated from 4-cyanopyridine and $\mathrm{B}_{2}(\mathrm{pin})_{2}$ played an important role in the catalytic cycle, which not only activates $\mathrm{C}-\mathrm{X}$ bond homolysis but also serves as a pyridine precursor. The selective cross-coupling of the resulting alkyl radicals generated from the addition reaction of $\mathrm{RCF}_{2}$ radicals to alkenes and 4-cyanopyridineboryl radicals delivered the corresponding product with a quaternary carbon center.

In 2019, another three-component reaction of fluoroalkyl halides, alkenes and arenes was reported by the Liang group. ${ }^{83}$ An unprecedented three-component direct $\mathrm{C}-\mathrm{H}$ addition was achieved in the challenging meta-selective fashion catalyzed by $\mathrm{Ru}$. Control experiments and computational analyses were carried out to investigate the mechanism. The plausible catalytic cycle is outlined in Scheme 25. Initially, carboxylateassisted $\mathrm{C}-\mathrm{H}$ ruthenation occurred to give 46, which was followed by the reaction with fluoroalkyl halide and alkenes via SET to afford 47 and radical 48. Subsequently, the newly formed radical bond added to the para-position of the $\mathrm{C}-\mathrm{Ru}$ bond, leading to species 49 , which was followed by rearomatization to give ruthenacycle 50. Finally, the meta-functionali-

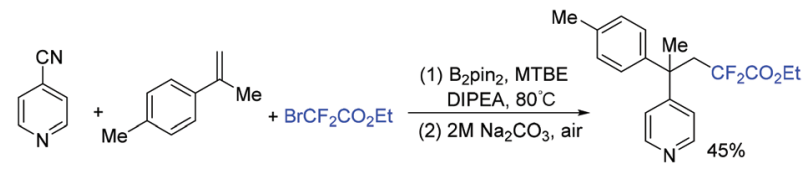

Scheme 24 The perfluoroalkylative pyridylation of alkenes. 


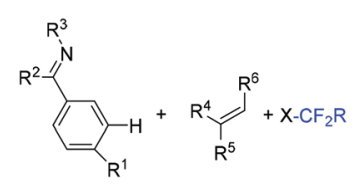

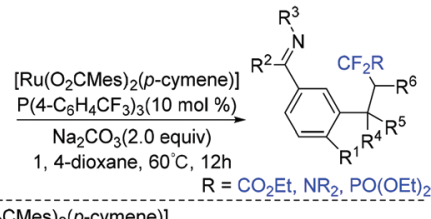<smiles>Cc1cccc(-c2ccccn2)c1</smiles>
$\left[\mathrm{Ru}\left(\mathrm{O}_{2} \mathrm{CMes}\right)_{2}(p\right.$-cymene $\left.)\right]$

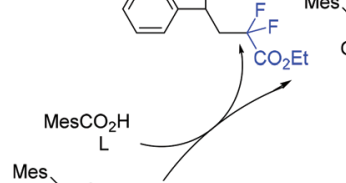

Mes.<smiles>C[R1]1(C)OCO1</smiles>

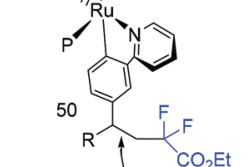

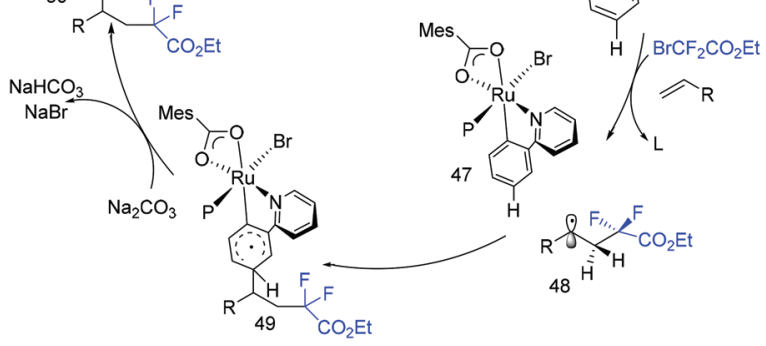

Scheme 25 The three-component reaction of fluoroalkyl halides, alkenes and arenes.

zation product was delivered by demetalation, with the release of ruthenium(II) complex.

In 2018, the Li group found that the 1,2-alkylarylation reaction of styrenes, $\alpha$-carbonylalkyl bromides and $N, N$-disubstituted anilines could be conducted in the presence of photoredox and copper cooperative catalysis (Scheme 26). ${ }^{84}$ In addition to Friedel-Craftss alkylation, this reaction provided a new access for the $\mathrm{C}-\mathrm{H}$ alkylation of $\mathrm{N}, \mathrm{N}$-disubstituted anilines with high para-selectivity. Carbonylalkyl bromides such as primary, secondary and tertiary $\alpha$-bromoalkyl ketone esters, malonic esters and cycloalkane were all good partners for this reaction. The product with $79 \%$ yield was obtained when $\mathrm{BrCF}_{2} \mathrm{CO}_{2}$ Et was employed. $\mathrm{CF}_{2} \mathrm{CO}_{2}$ Et radical which was generated from the reduction of $\mathrm{BrCF}_{2} \mathrm{CO}_{2} \mathrm{Et}$ with the excited state $\mathrm{Ru}(\mathrm{bpy})_{3}{ }^{* 2+}$ was proposed in the mechanism.

Recently, the asymmetric three-component Minisci reaction of quinolines or pyridines with $\alpha$-bromocarbonyl compounds

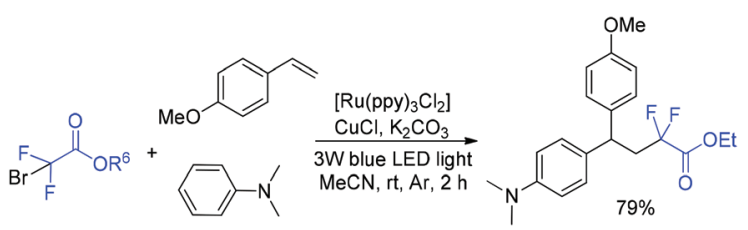

Scheme $26 \mathrm{C}(\mathrm{sp} 3)-\mathrm{Br} / \mathrm{C}(\mathrm{sp} 2)-\mathrm{H}$ functionalization mediated by photoredox and copper cooperative catalysis.

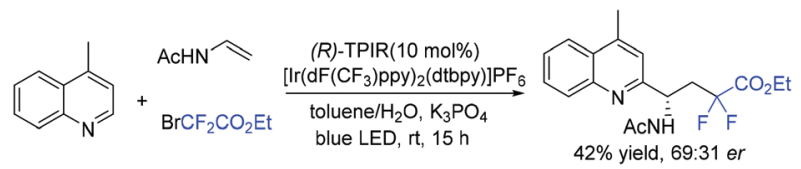

Scheme 27 Alkenes for difluoromethyl radical addition.

and enamides mediated by dual photoredox and chiral Brønsted acid catalysis was presented by Studer and coworkers (Scheme 27). ${ }^{85}$ A range of valuable chiral $\gamma$-amino acid derivatives are obtained with good to excellent enantioselectivities. But when $\mathrm{BrCF}_{2} \mathrm{CO}_{2} \mathrm{Et}$ was used in this system, only $42 \%$ yields and 69:31 er value was isolated.

The group of $\mathrm{Li}$ and Han demonstrated that N-heterocyclic carbene (NHC) could also catalyze radical difluoroalkylation of olefins. ${ }^{86}$ The dearomative difunctionalization of indoles could be readily achieved via this reaction. The mechanism is presented in Scheme 28. Firstly, the reaction of NHC catalyst, aldehyde and base occurred to give Breslow intermediate 51, which was followed by SET with fluoroalkyl reagent $\mathrm{RCF}_{2} \mathrm{X}$ to afford fluoroalkyl radical and a persistent ketyl radical 55. Subsequently, radical 54 generated from the addition of fluoroalkyl radical to styrene was trapped by radical 55 through a radical-radical cross-coupling pathway to form intermediate F. Finally, the fluoroketone product was obtained with the release of NHC.

\subsection{Hydrodifluoroalkylation with alkenes}

In 2018, visible-light-induced 1,6-difunctionalizations of alkenes with $\mathrm{BrCF}_{2} \mathrm{CO}_{2} \mathrm{Et}$ were realized by the Nevado group

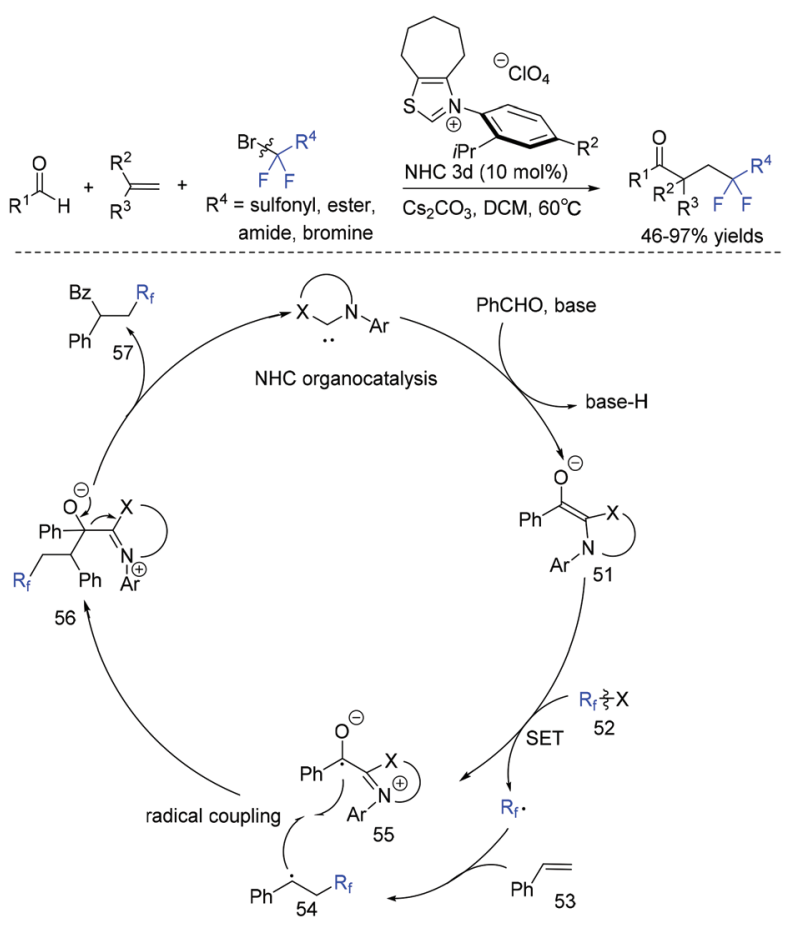

Scheme 28 The N-heterocyclic carbene-catalyzed radical difluoroalkylation of olefins. 


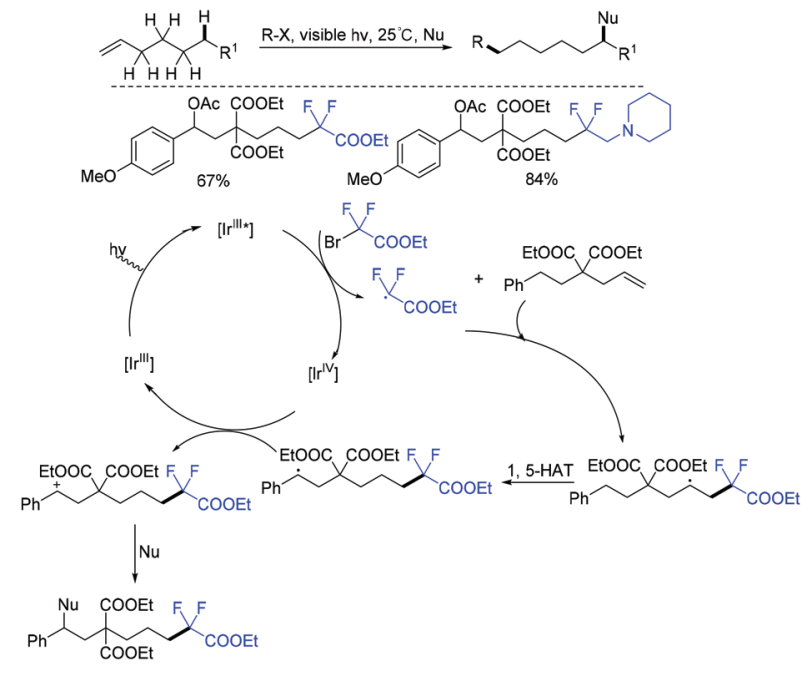

Scheme 29 The visible-light-induced 1,6-difunctionalizations alkenes.

(Scheme 29). ${ }^{87}$ The addition reaction of $\mathrm{CF}_{2} \mathrm{CO}_{2} \mathrm{Et}$ radical which was generated from the reduction of $\mathrm{BrCF}_{2} \mathrm{CO}_{2} \mathrm{Et}$ by $[\mathrm{Ir}]^{\mathrm{III} *}$ with double bond afforded a vicinal radical intermediate. Subsequently, a remote benzylic radical was generated via 1,5-HAT, which can be trapped with $\mathrm{O}$ - or C-nucleophiles to produce new $\mathrm{Csp} 3-\mathrm{O}$ and $\mathrm{Csp} 3-\mathrm{Csp} 2$ bonds at room temperature.

In the same year, a series of difluoroalkylated ketones or aldehydes was obtained by the group of Luo and Cheng via $\mathrm{Cu}$-catalyzed oxidation of alcohols with $\mathrm{BrCF}_{2} \mathrm{CO}_{2} \mathrm{Et}$ (Scheme 30). ${ }^{88}$ The catalytic oxidation of alcohols and difluoroalkylation of alkenes were concurrently realized in this reaction, enabling a highly efficient and attractive method for organic synthesis. Similarly, radical difluoroalkylation of alkenes, intramolecular 1,5- or 1,6-HAT, SET oxidation and deprotonation reactions were proposed in the mechanism.

Besides alcohols, the group of $\mathrm{Ma}$ and Li demonstrated that difluoroalkylated ketones could also be synthesized via visiblelight-mediated cascade remote oxyfluoroalkylation of alkenes employing dimethyl sulfoxide as the oxidant and solvent (Scheme 31). ${ }^{89}$ From the plausible mechanism we can see that the alkene fluoroalkylation-initiated remote benzyl $\mathrm{C}-\mathrm{H}$ bond activation via 1,5-H migration in a highly controlled site-selec-

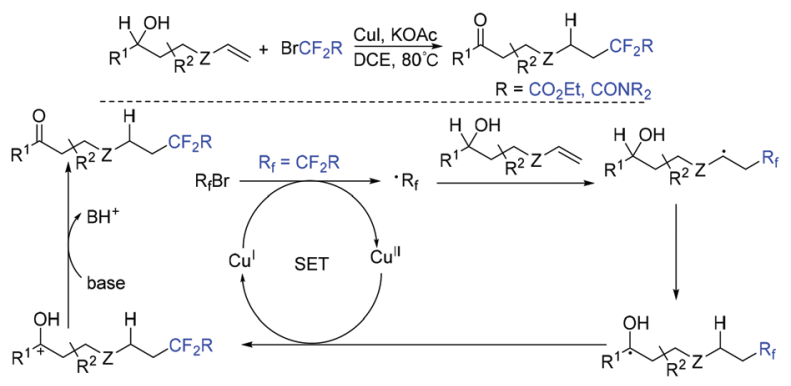

Scheme 30 The $\mathrm{Cu}$-catalyzed oxidation of alcohols with $\mathrm{BrCF}_{2} \mathrm{CO}_{2} \mathrm{Et}$.

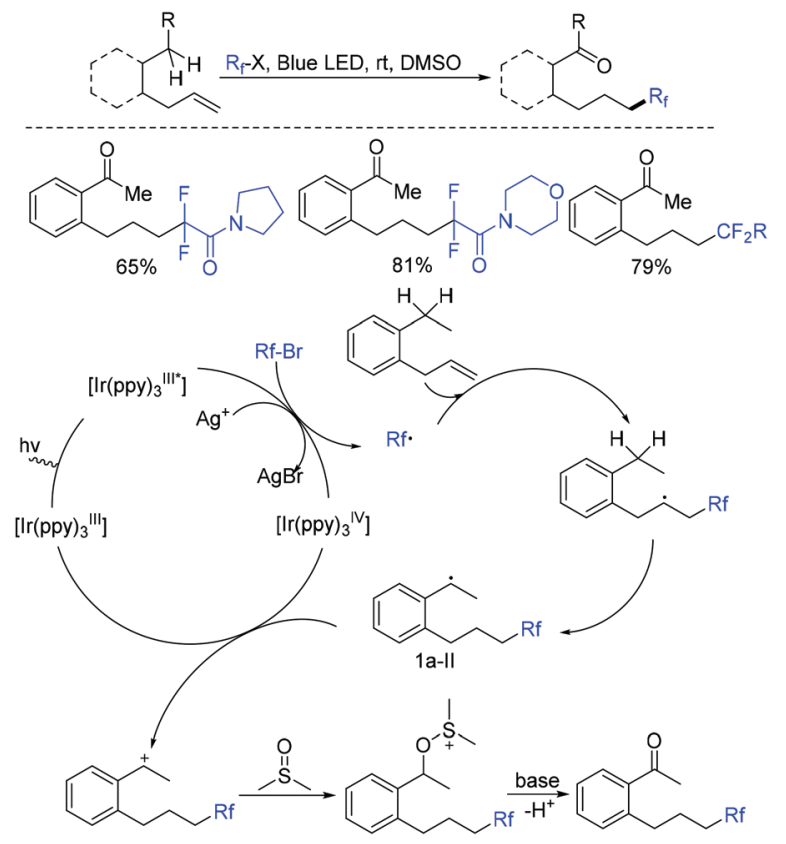

Scheme 31 The Cu-catalyzed oxidation of alcohols with $\mathrm{BrCF}_{2} \mathrm{CO}_{2} \mathrm{Et}$.

tive manner and Kornblum reaction with dimethyl sulfoxide as the oxidant played a key role in this reaction.

The group of Zhou and Chen realized the reaction of methylene-2-oxazolines with difluoroalkylating reagents via a photoredox strategy (Scheme 32). ${ }^{90}$ Difluoroalkylated oxazoles with diverse functionalities were prepared using this method. Tandem radical addition of $\mathrm{CF}_{2} \mathrm{CO}_{2}$ Et radical and subsequent oxidative aromatization steps were proposed in the reaction mechanism.

The photoredox-catalyzed reaction of $\alpha, \beta$-dehydroamino acids and peptides with $\mathrm{BrCF}_{2} \mathrm{CO}_{2}$ Et was demonstrated by the Mancheño group, providing various of non-natural amino acids and peptides (Scheme 33). ${ }^{91}$ Additionally, arylsulfonyl chlorides or various $N$-(acyloxy)phthalimides (NHPI esters) were also effective for this reaction. In the catalytic cycle, $\mathrm{PC}^{*}$ was reduced by Hantzsch ester to form $\mathrm{PC}^{*-}$ and HE-radical cation. Then $\mathrm{PC}^{*-}$ reacted with $\mathrm{BrCF}_{2} \mathrm{CO}_{2} \mathrm{Et}$ to produce $\mathrm{CF}_{2} \mathrm{CO}_{2}$ Et radical and the photocatalyst PC. Subsequently, the addition reaction of $\mathrm{CF}_{2} \mathrm{CO}_{2}$ Et radical and the double bond of the $\alpha, \beta$-dehydroamino acid resulted in radical $\mathrm{I}$, which was followed by reduction and protonation reaction to provide the desired product.

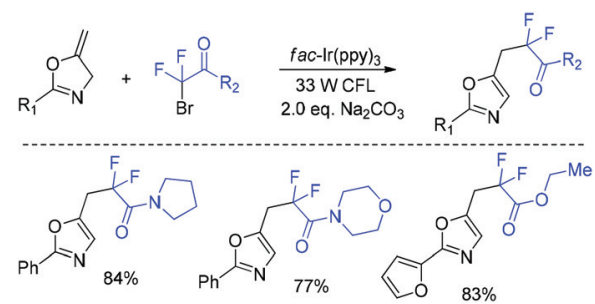

Scheme 32 The difluoroalkylation of alkenes. 


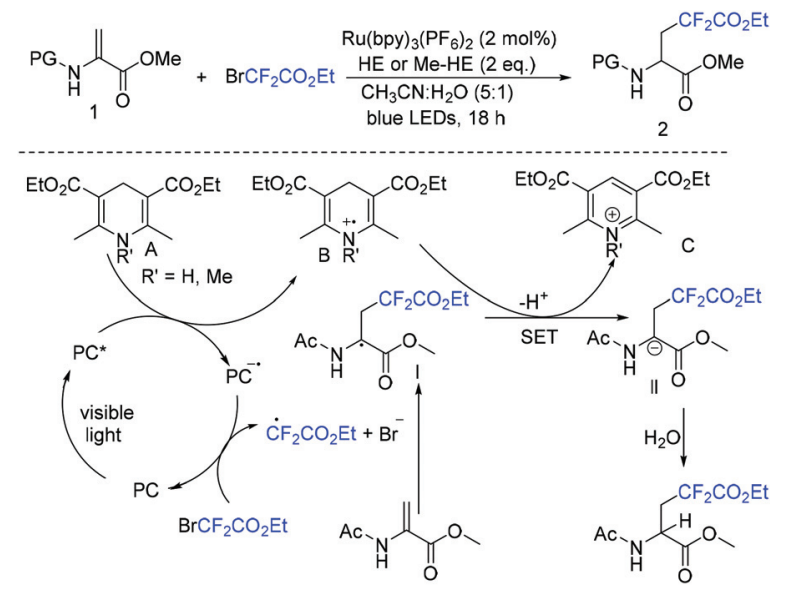

Scheme 33 The photoredox-catalyzed reaction of $\alpha, \beta$-dehydroamino acids and peptides with $\mathrm{BrCF}_{2} \mathrm{CO}_{2} \mathrm{Et}$.

\subsection{Heterodifluoroalkylation with alkenes}

Due to its thermodynamic stability and kinetic inertness, it is challenging to utilize $\mathrm{CO}_{2}$ for organic synthesis. Many methods have been developed to harness $\mathrm{CO}_{2}$ for the synthesis of various organic compounds up to now. ${ }^{92-99}$ In 2018, a selective difluoroalkylation of allylamines with carbon dioxide via visible-light photoredox catalysis was developed by the $\mathrm{Yu}$ group. ${ }^{100}$ Various 2-oxazolidinones with difluoroalkyl groups were generated. A plausible mechanism is outlined in Scheme 34. $\mathrm{Ru}(\mathrm{I})$ which was generated from the reductive quenching of excited photocatalyst $\mathrm{Ru}(\mathrm{II})^{*}$ by DABCO reduces $\mathrm{BrCF}_{2} \mathrm{R}$ to $\mathrm{CF}_{2} \mathrm{R}$ radical. Then carbamate 58 which was generated from 59 and $\mathrm{CO}_{2}$ in situ reacted with $\mathrm{CF}_{2} \mathrm{R}$ radical to give benzylic radical 60 . Then oxidation of 60 by excited $\mathrm{Ru}(\mathrm{II})^{*}$ and intramolecular cyclization of $\mathbf{6 1}$ occurred in sequence to afford the final product $\mathbf{6 2}$.

The cyclization of alkenyl carboxylic acids with difluoroalkyl reagents for the synthesis of difluoroalkylated lactones has

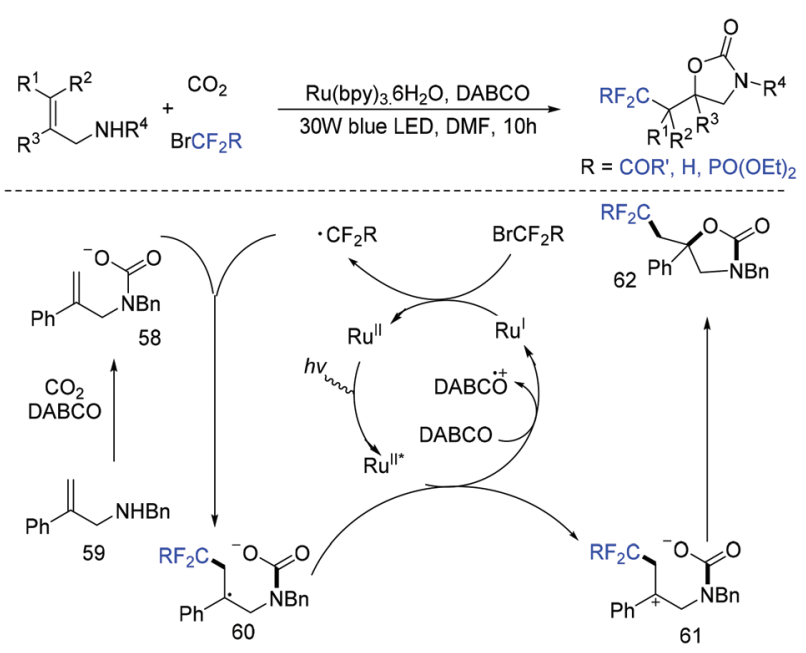

Scheme 34 The difluoroalkylation of allylamines with carbon dioxide.

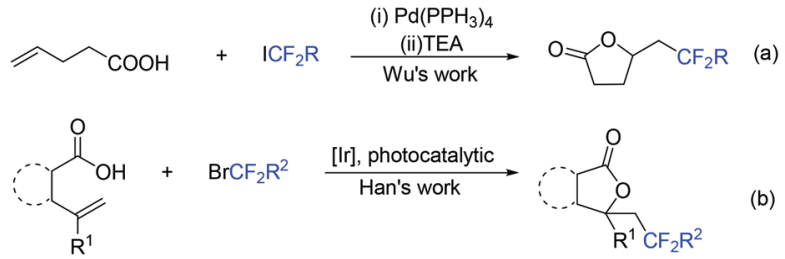

Scheme 35 The cyclization of alkenyl carboxylic acids with difluoroalkyl reagents.

been established in recent years. The Wu group developed a palladium-catalyzed reaction for the synthesis of difluoroalkylated lactones (Scheme 35a). ${ }^{101}$ In 2017, the iridium-catalyzed photoredox lactonization for the synthesis of difluoroalkylated lactones was realized by the Han group (Scheme 35b). ${ }^{102}$ In 2018, the group of Li realized the copper(I)-catalyzed difluoroalkylation of alkenyl carboxylic acids under mild reaction conditions (Scheme 36). ${ }^{103}$ The highly valued $\mathrm{CF}_{2}$-containing lactones were delivered in moderate to excellent yields. A radical-involved pathway was proposed for this catalytic reaction. Firstly, an initial SET from Cu(I)Ln 63 to $\mathrm{RCF}_{2} \mathrm{Br}$ occurred to generate $\mathrm{RCF}_{2}$ radical and $\mathrm{Cu}(\mathrm{II})$. Subsequently, the addition reaction of $\mathrm{RCF}_{2}$ radical to unsaturated carboxylic acid delivered the difluoroalkyl radical intermediate 64 . Then, the carbocation intermediate 65 was formed by oxidation of 64 , concurrently regenerating the active copper(I) catalyst. Finally, the desired product was obtained through an intramolecular nucleophilic attack (Scheme 36, path a). Another pathway involving the $\mathrm{Cu}(\mathrm{III})$ intermediate $\mathbf{6 6}$ followed by the reductive elimination under basic conditions to generate the final product was also possible (Scheme 36, path b). In the same

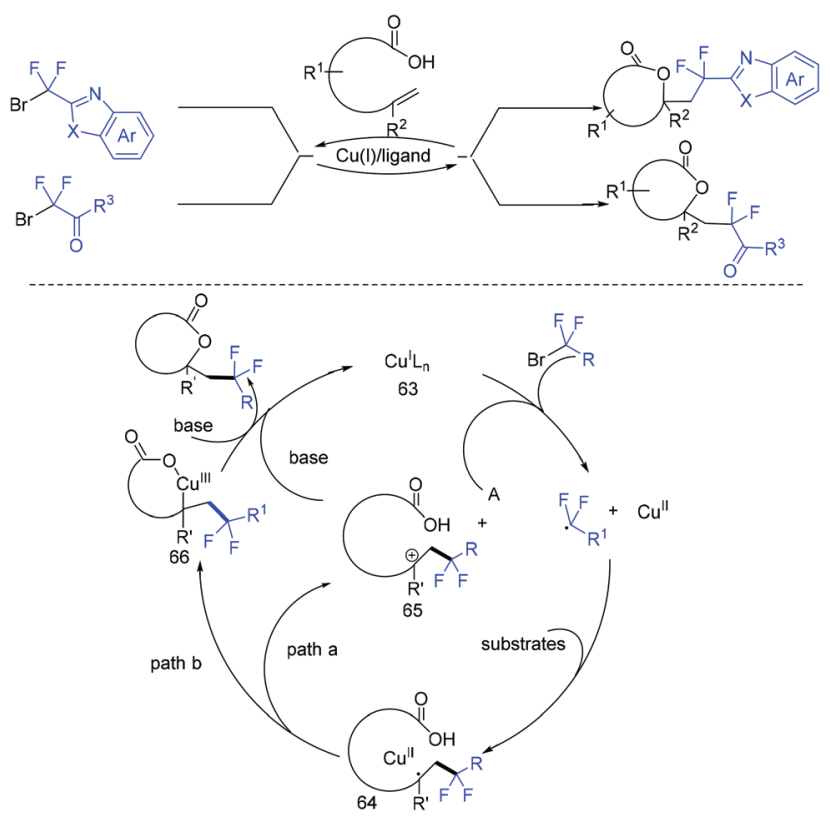

Scheme 36 The copper-catalyzed cyclization of alkenyl carboxylic acids with difluoroalkyl reagents. 

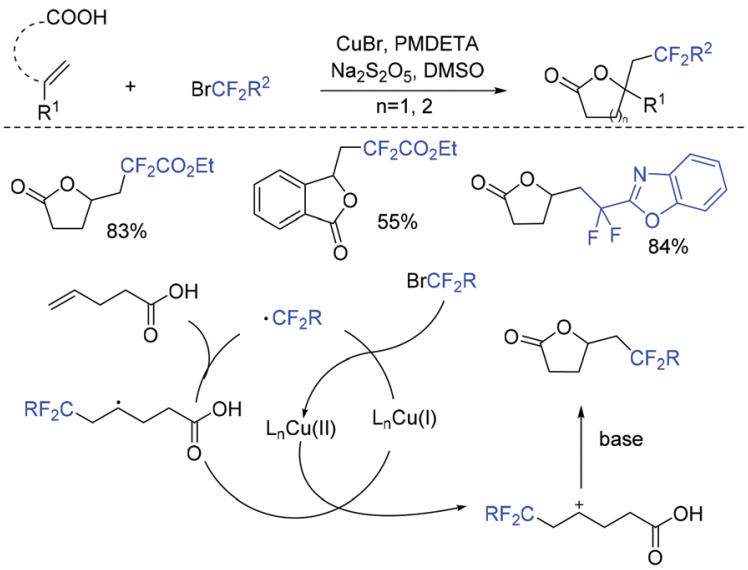

Scheme 37 The copper-catalyzed cyclization of unsaturated carboxylic acids.

year, another copper-catalyzed difluoroalkylation of alkenyl carboxylic acids for the synthesis of lactone was also reported by the Wang group (Scheme 37). ${ }^{104} \mathrm{~A} \mathrm{Cu}(\mathrm{I}) / \mathrm{Cu}(\mathrm{II})$ mechanism was suggested for this transformation.

The Yang group demonstrated that bromodifluoromethylphosphonates are also good reagents for the construction of difluoroalkylated lactones (Scheme 38). ${ }^{105}$ The reaction of bromodifluoromethylphosphonates with alkenyl acids was smoothly carried out in the presence of visible light. Besides acids, alcohols and sulfonamides were also suitable for this system. $\mathrm{CF}_{2} \mathrm{P}$-containing heterocycles can be obtained efficiently. Similar to the production process of $\mathrm{RCF}_{2}$ radical, the oxidative quenching process of the excited $f a c$ - $\left[\operatorname{Ir}(\mathrm{ppy})_{3}\right]^{*}$ with diisopropyl bromodifluoromethylphosphate via SET to afford $\mathrm{CF}_{2} \mathrm{PO}\left(\mathrm{O}^{\mathrm{i}} \mathrm{Pr}\right)_{2}$ radical was involved in the reaction mechanism.

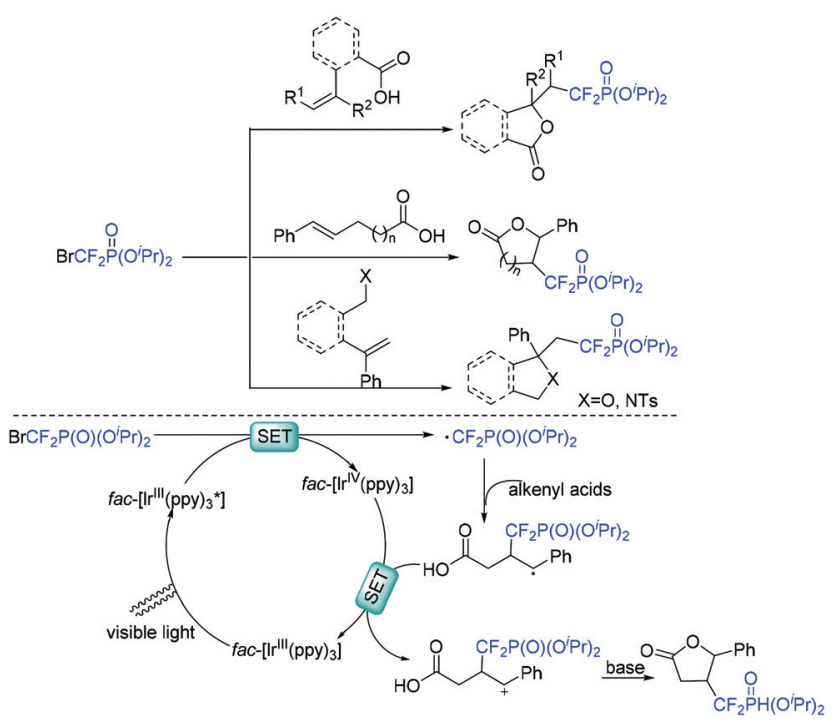

Scheme 38 The reaction of bromodifluoromethylphosphonates with alkenyl acids.
Copper-catalyzed intramolecular oxydifluoroalkylation of hydroxyl-containing alkenes for the synthesis of various fluoroalkylated tetrahydrofurans was also described by the group of Wang and Guo. ${ }^{106}$ Besides ethyl bromodifluoroacetate, other functionalized difluoroalkylated bromides were also well employed in this system. The proposed mechanism of this reaction is shown in Scheme 39. First, $\mathrm{RCF}_{2}$ radical 67 and $\mathrm{Cu}$ (II) intermediate were generated from the oxidation of $\mathrm{Cu}(\mathrm{I})$ by ethyl bromodifluoroacetate. The addition reaction of radical 67 and alkenes occurred to generate intermediate 68, which was trapped by $\mathrm{Cu}$ (II) in the presence of base $\mathrm{Na}_{2} \mathrm{CO}_{3}$ to form intermediate 69. Then two possible intermediates 70 or $\mathbf{7 1}$ may be formed. The desired product can be generated from 70 via nucleophilic attack of activated alcohol toward carbon cation species. Alternatively, intermediate $\mathbf{7 1}$ which undergoes reductive elimination could also deliver the desired product. These two pathways are possible simultaneously. In 2019, this group demonstrated that when amine-containing olefins were used as substrates instead of hydroxyl-containing alkenes, diverse difluorinated nitrogen-containing polycycles were obtained (Scheme 40). ${ }^{107}$ Oxidation of $\mathrm{Cu}(\mathrm{I})$ species by $\mathrm{BrCF}_{2} \mathrm{CO}_{2} \mathrm{Et}$ through a SET to afford an electrophilic $\mathrm{RCF}_{2}$ radical and $\mathrm{Cu}$ (II) species was also described in the proposed mechanism. Recently, the photoredox-catalyzed oxydifluoroalkylative cyclization of alkenes using $\mathrm{R}_{\mathrm{f}} \mathrm{I}$ was also reported by the Luo group. ${ }^{108}$

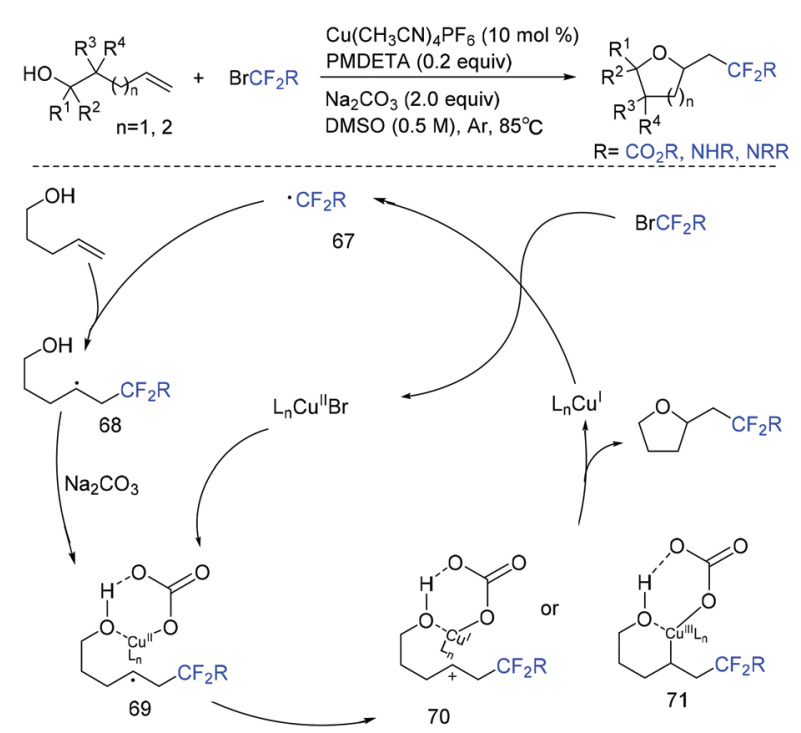

Scheme 39 The copper-catalyzed intramolecular oxydifluoroalkylation of hydroxyl-containing alkenes.

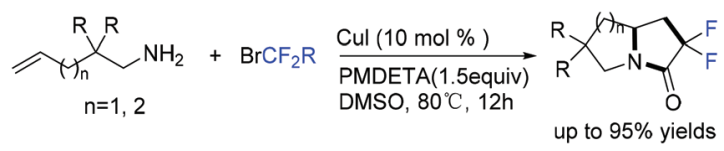

Scheme 40 The synthesis of difluorinated nitrogen-containing polycycles. 


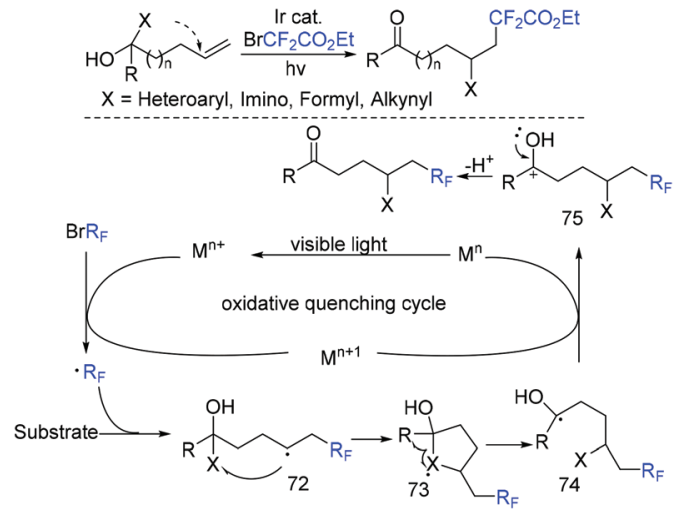

Scheme 41 The synthesis of difluorinated alkyl ketones.

Distal functional group migration was also observed in the reaction of alkenes with $\mathrm{BrCF}_{2} \mathrm{CO}_{2} \mathrm{Et}$ (Scheme 41). ${ }^{109}$ In combination with visible-light photocatalysis, difluorinated alkyl ketones were readily obtained with high yields. Besides heteroaryl group, imino, formyl, and alkynyl groups were also well tolerated in this system. Radical addition, functional group migration and oxidation reaction were proposed in the plausible mechanism.

The Song group found that the copper/ $\mathrm{B}_{2} \mathrm{pin}_{2}$ system was effective for difluoroalkylation-thiolation of arylalkenes (Scheme 42). ${ }^{110} \mathrm{~B}_{2} \mathrm{pin}_{2}$ as an organic reductant played an important role in this transformation, which enables the simultaneous installation of a $\mathrm{C}(\mathrm{sp} 3)-\mathrm{C}\left(\mathrm{F}_{2} \mathrm{R}\right)$ bond and a $\mathrm{C}(\mathrm{sp} 3)-\mathrm{S}(\mathrm{R})$ bond by utilizing two electrophilic substrates. Radical trapping experiments suggested that the $\mathrm{CF}_{2} \mathrm{CO}_{2} \mathrm{Et}$ radical was involved in the reaction mechanism.

The three-component difluoroalkylation-peroxidation of alkenes with difluorohaloactates and hydroperoxides catalyzed by $\mathrm{Co}(\mathrm{acac})_{2}$ was developed by the Li group (Scheme 43). ${ }^{111}$ Various $\beta$-peroxyldifluoroalkyl derivatives were accessed efficiently via this strategy. In addition, other halides were also tolerated in this reaction, giving the alkylation-peroxidation products. $\mathrm{CF}_{2} \mathrm{CO}_{2} \mathrm{Et}$ radical generated from the reduction of $\mathrm{BrCF}_{2} \mathrm{CO}_{2} \mathrm{Et}$ by $\mathrm{Co}(\mathrm{II})$ added to alkenes to form radical 76 . Then tert-butylperoxy radical or cobalt tert-butylperoxy 77 which were generated from the reaction of cobalt(III) species

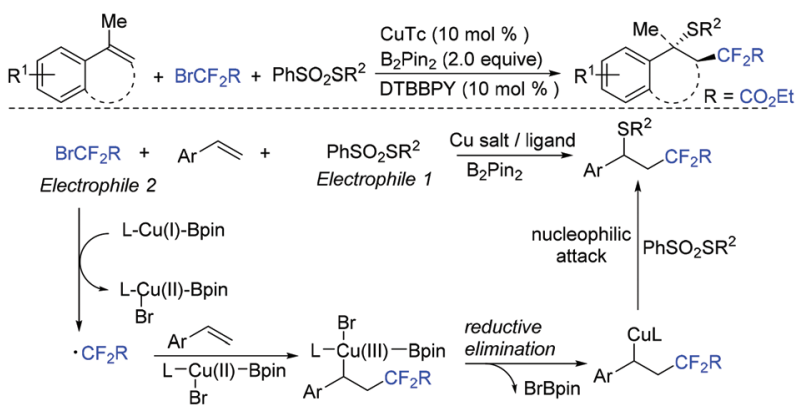

Scheme 42 The light-mediated Truce-Smiles rearrangement.

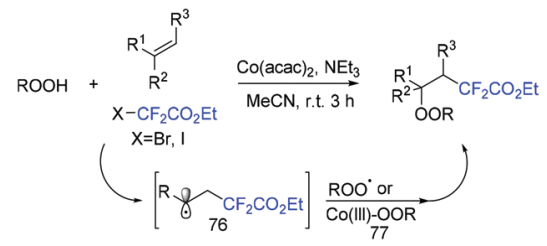

Scheme 43 The three-component difluoroalkylation-peroxidation of alkenes with difluorohaloactates and hydroperoxides.

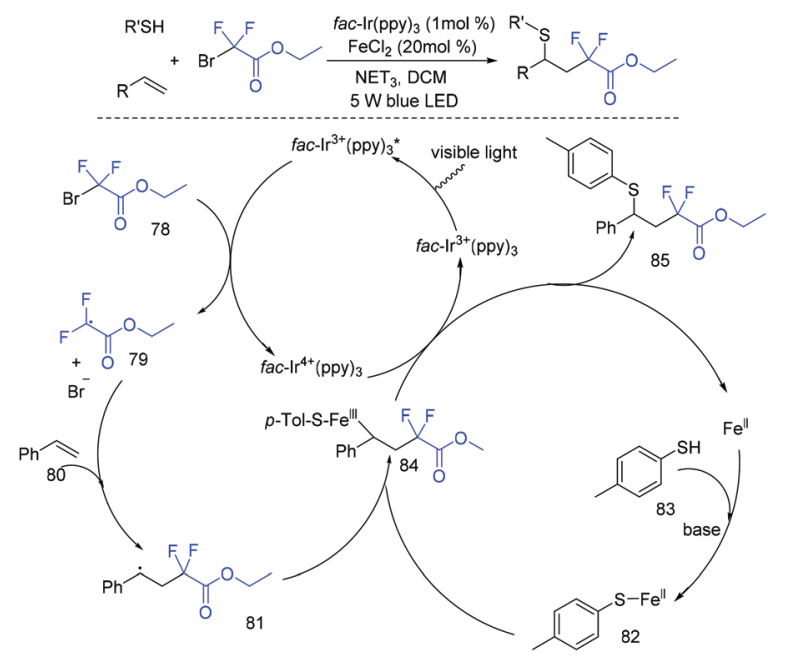

Scheme 44 The difluoroalkylation-thiolation of alkenes with $\mathrm{BrCF}_{2} \mathrm{CO}_{2} \mathrm{Et}$ and $\mathrm{RSH}$.

with hydroperoxide react with radical 76 to afford the desired product.

In 2019, the difluoroalkylation-thiolation of alkenes with $\mathrm{BrCF}_{2} \mathrm{CO}_{2} \mathrm{Et}$ and $\mathrm{RSH}$ mediated by iron was developed by the Cai group (Scheme 44). ${ }^{112}$ Csp3-Csp3 and Csp3-S bonds were simultaneously formed in this reaction. $\mathrm{FeCl}_{2}$ which can be used to activate thiols via Fe/S complex has been documented. ${ }^{113}$ A possible reaction mechanism was proposed as shown in Scheme 44. A radical intermediate 79 and an oxidized photocatalyst $f a c-\mathrm{Ir}^{4+}(\mathrm{ppy})_{3}$ were generated from the oxidation of $f a c-\mathrm{Ir}^{3+}(\mathrm{ppy})_{3}{ }^{*}$ with $\mathrm{BrCF}_{2} \mathrm{CO}_{2}$ Et. Then the radical 79 added to styrene 80, giving a benzyl radical intermediate $\mathbf{8 1}$. On the other hand, the reaction of $\mathrm{Fe}(\mathrm{II})$ with 4-methylbenzenethiol delivered an iron-sulfur intermediate 82 in the presence of a base, which was followed by recombination with $\mathbf{8 1}$ to produce intermediate 84. Finally, a SET occurred between 84 and fac$\operatorname{Ir}^{4+}(\text { ppy })_{3}$, providing the desired product, along with the liberation of $\mathrm{FeCl}_{2}$. In the same year, the same group ${ }^{114}$ found that three-component difluoroalkylamination of alkenes with $\mathrm{BrCF}_{2} \mathrm{CO}_{2} \mathrm{Et}$ and amines could also be realized under similar reaction conditions (Scheme 45). Besides thiols and amines, alcohol was also found to be suitable for this type of reaction by the Nishikata group (Scheme 46). ${ }^{115}$ The copper-mediated radical and cation crossover mechanism was involved in the reaction pathway. 


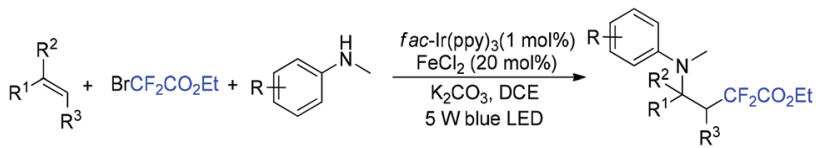

Scheme 45 The difluoroalkylation-thiolation of alkenes with $\mathrm{BrCF}_{2} \mathrm{CO}_{2} \mathrm{Et}$ and amines.

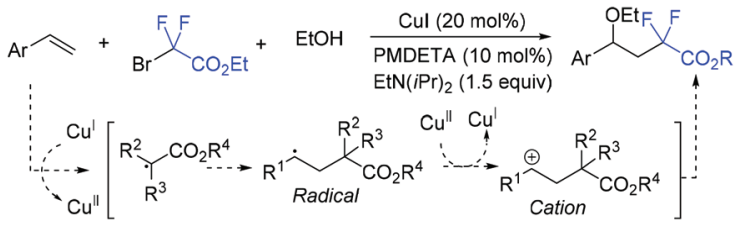

with $\mathrm{BrCF}_{2} \mathrm{CO}_{2} \mathrm{Et}$ and alcohols.

1,4-Diazabicyclo[2.2.2] octane-bis(sulfur dioxide) adduct (DABSO) as an inexpensive and readily available reagent has been widely used in organic transformations. ${ }^{116-118}$ In 2019, Chen and coworkers achieved a zinc-mediated intermolecular reductive radical fluoroalkylsulfination of unsaturated $\mathrm{C}-\mathrm{C}$ bonds with fluoroalkyl bromides and DABSO (Scheme 47). ${ }^{119}$ Alkenes and alkynes were all tolerated in this reaction, affording the product with moderate yields. Preliminary mechanistic investigations revealed that a sulfur dioxide anion radical species reduced by $\mathrm{Zn}$ from $\mathrm{SO}_{2}$ was involved in this reaction.

Due to side reactions, nickel-catalyzed dialkylation of alkenes remains underdeveloped. In 2019, a nickel-catalyzed three-component difluoroalkylation-alkylation of $N$-vinyl-2-pyrrolidinone with difluoroalkyl bromides and dialkylzinc reagents was reported by the Zhang group (Scheme 48). ${ }^{120}$ $N$-Vinyloxazolidinone and $N$-vinylacetamide can also be applied to this system. $\mathrm{BrCF}_{2} \mathrm{COOEt}$ was used for this reaction, the corresponding product being obtained in 51\% yield. Two mechanisms initiated by $\mathrm{Ni}$ (I) or Ni(II) were possible for this reaction. As a key intermediate, radical 86 was generated in both mechanisms.

The Shi group found that $\mathrm{CF}_{2} \mathrm{CO}_{2} \mathrm{Et}$ radical was also a good partner to react with alkenyl diboronate complexes (Scheme 49). ${ }^{121}$ In the presence of Ru photocatalyst, gem-bis (boryl)alkanes were formed from the reaction of alkenyl dibor-

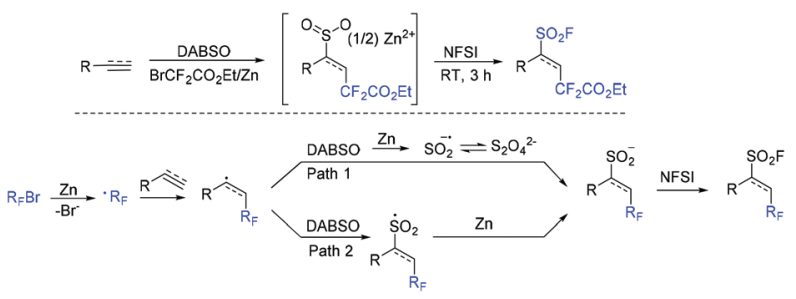

Scheme 47 The difluoroalkylation-thiolation of alkenes with $\mathrm{BrCF}_{2} \mathrm{CO}_{2} \mathrm{Et}$ and alcohols.
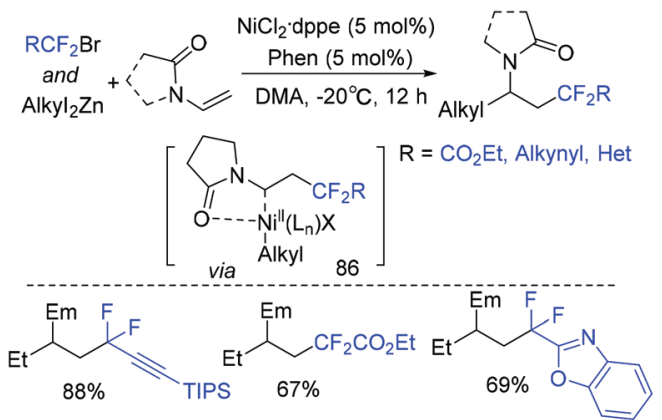

Scheme 48 The difluoroalkylation-thiolation of alkenes with $\mathrm{BrCF}_{2} \mathrm{CO}_{2}$ Et and alcohols.

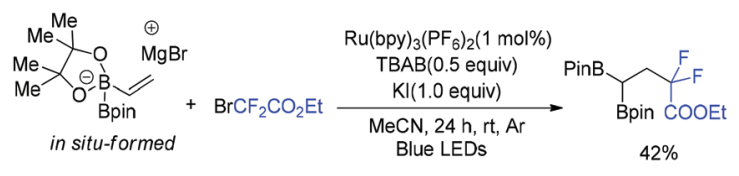

Scheme 49 The reaction of alkenyl diboronate complexes with $\mathrm{BrCF}_{2} \mathrm{CO}_{2} \mathrm{Et}$.

onate with diverse alkyl halides. Alkyl radicals added efficiently to the alkenyl diboronate complexes. Then the adduct radical anions undergo radical-polar crossover and 1,2-boryl-anion shift from boron to the $\alpha$-carbon to afford the final product.

In 2018, the difluoroacetylation of alkenes using ethyl bromodifluoroacetate $\left(\mathrm{BrCF}_{2} \mathrm{CO}_{2} \mathrm{Et}\right)$ catalyzed by copper was reported by the Zhu group (Scheme 50). ${ }^{122}$ In the presence of a base, $\mathrm{BrCF}_{2} \mathrm{CO}_{2}$ Et was reduced by the complex of $\mathrm{CuI} / \mathrm{B}_{2} \mathrm{Pin}_{2}$ to generate $\mathrm{CF}_{2} \mathrm{COOEt}$ radical 91 along with $\mathrm{Cu}(\mathrm{II})$ 92. Then, radical 91 added to the terminal carbon of alkenes to give carbon radical intermediate $\mathrm{E}$, which can be oxidized by $\mathbf{9 2}$, trapped by bromide ion to afford the desired product. Alternatively, intermediate $\mathbf{9 3}$ coupled with $\mathbf{9 2}$ to afford $\mathrm{Cu}$ (III) complex which was followed by reductive elimination to afford product with the release of $\mathrm{Cu}(\mathrm{I})$. This pathway was also possible.

The He group demonstrated that organophosphine was a good catalyst for atom transfer radical addition of alkenes with ethyl iododifluoroacetate (Scheme 51). ${ }^{123}$ Substrate-controlled selectivity was observed in this difluoroacetylation. When styrenes were subjected to this system, a Heck-type difluoroalkylated product was furnished. Difluoroalkylated alkanes were obtained when alkenes were used. A noncovalent interaction between the phosphine catalyst and carbon-iodine bond was suggested for this reaction.

The base-controlled selective (phenylsulfonyl)difluoromethylation of alkenes with $\mathrm{PhSO}_{2} \mathrm{CF}_{2} \mathrm{I}$ was observed by the Wang group (Scheme 52). ${ }^{124}$ Both atom transfer radical addition and Heck-type products could be obtained via simply tuning the organic or inorganic bases in the presence of visible light. The oxidative quenching of ${ }^{*} \mathrm{Ru}(\mathrm{bpy})^{2+}$ with $\mathrm{PhSO}_{2} \mathrm{CF}_{2} \mathrm{I}$ was proposed in the mechanism. Both types of products can be formed via two pathways. 


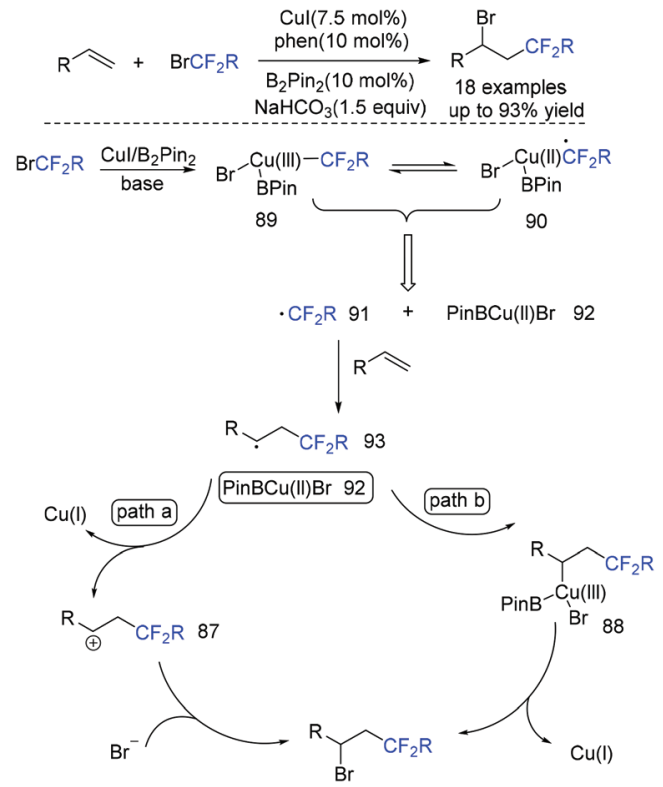

Scheme 50 The copper-catalyzed difluoroacetylation of alkenes.

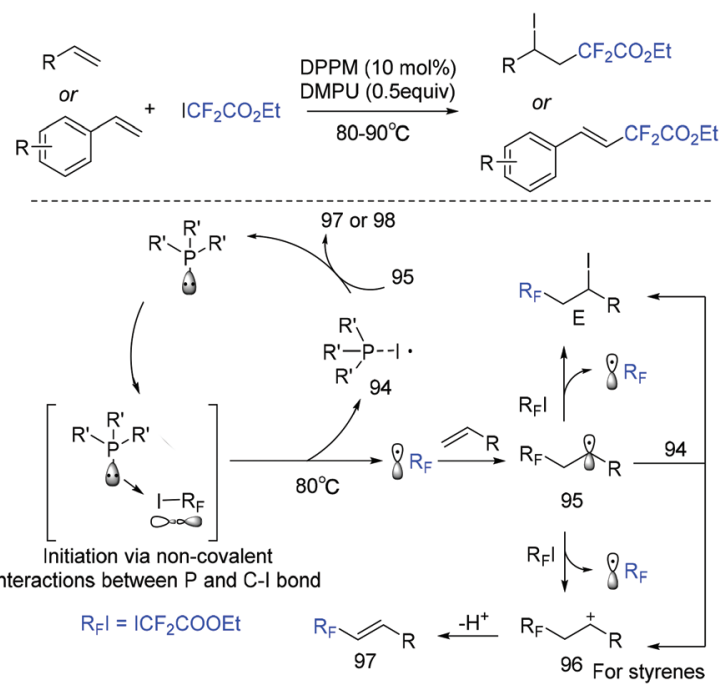

Scheme 51 The organophosphine-catalyzed difluoroalkylation of alkenes.

The photoredox-catalyzed oxydifluoroalkylation of alkenes with $\mathrm{BrCF}_{2} \mathrm{CO}_{2} \mathrm{R}$ using dimethyl sulfoxide (DMSO) as an oxidant was reported by Tang and coworkers (Scheme 53). ${ }^{125}$ From the proposed mechanism we can see that AgTFA as the additive assisted the formation of $\mathrm{CF}_{2} \mathrm{CO}_{2} \mathrm{R}$ radical via oxidative quenching of ${ }^{*} \operatorname{Ir}(\mathrm{ppy})_{3}$ with $\mathrm{BrCF}_{2} \mathrm{CO}_{2} \mathrm{R}$. The final products were isolated via Kornblum oxidation of carbocation 100 with DMSO.

\subsection{Other difluoroalkylation with alkenes}

The palladium-mediated vinylic $\mathrm{C}-\mathrm{H}$ difluoroalkylation of ketene dithioacetals with $\mathrm{BrCF}_{2} \mathrm{CO}_{2}$ Et was realized by the
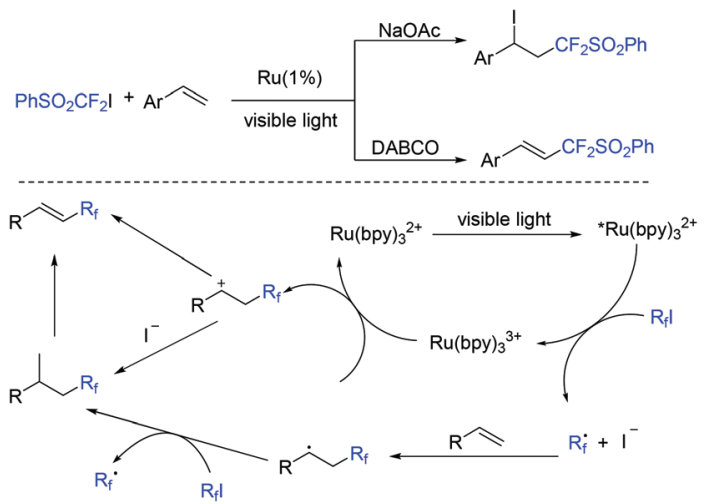

Scheme 52 The (phenylsulfonyl)difluoromethylation of alkenes with $\mathrm{PhSO}_{2} \mathrm{CF}_{2} \mathrm{I}$.
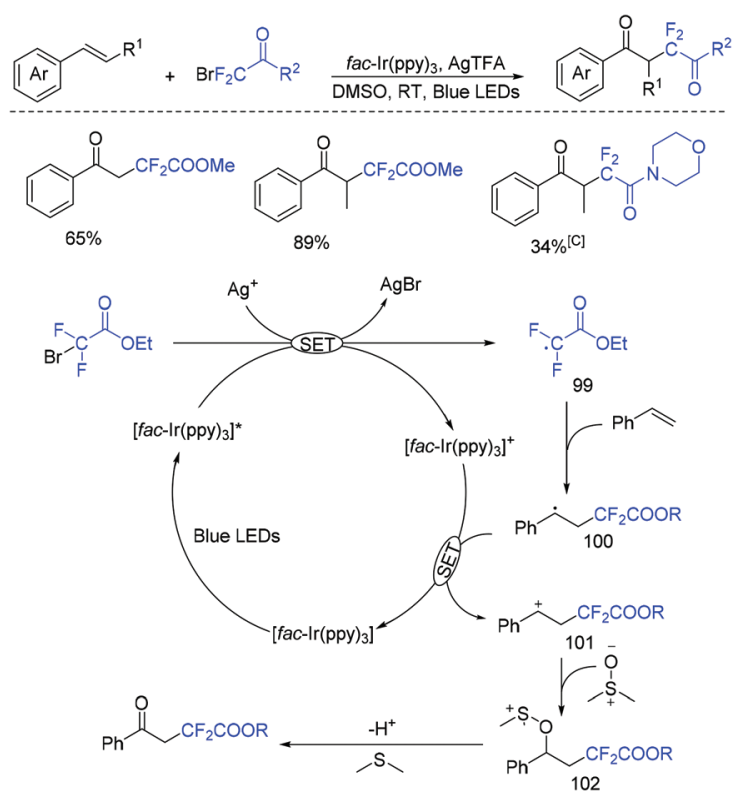

Scheme 53 The photoredox-catalyzed oxydifluoroalkylation of alkenes with $\mathrm{BrCF}_{2} \mathrm{CO}_{2} \mathrm{R}$ using dimethyl sulfoxide.

group of Wang and Zhu in 2018 (Scheme 54). ${ }^{126}$ Two SET processes were involved in the reaction mechanism. Owing to the unique structural characters of ketene dithioacetals, a $\operatorname{Pd}(0) / \operatorname{Pd}(\mathrm{I})$ catalytic cycle was proposed for this reaction, which was different from the known palladium catalyzed Heck-type reaction of alkenes with fluoroalkyl bromides.

$\mathrm{Xu}$ and coworkers reported a visible-light-induced crosscoupling reaction of para-quinone methides ( $p$-QMs) with difluoroalkylating reagents (Scheme 55). ${ }^{127}$ A series of difluoroalkylated diarylmethane compounds with pharmaceutical potential was accessed. From the plausible mechanism we can see that the excited photocatalyst, ${ }^{*} f a c$ - $\operatorname{Ir}(\mathrm{ppy})_{3}$, was primarily quenched by $p$-QMs to afford the diarylmethane radical intermediates, which was followed by a radical-radical cross-coupling reaction with difluoroalkyl radicals to deliver the final product. Amine could react as reductant with both $\operatorname{Ir}(\mathrm{IV})$ and 


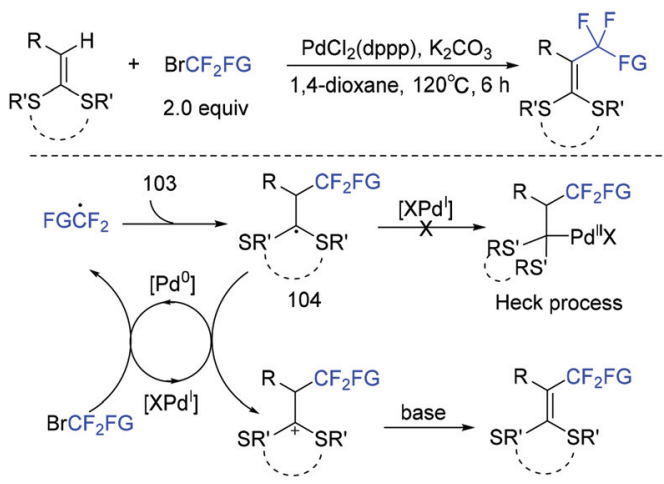

Scheme 54 The palladium-mediated $\mathrm{C}-\mathrm{H}$ difluoroalkylation of ketene dithioacetals.
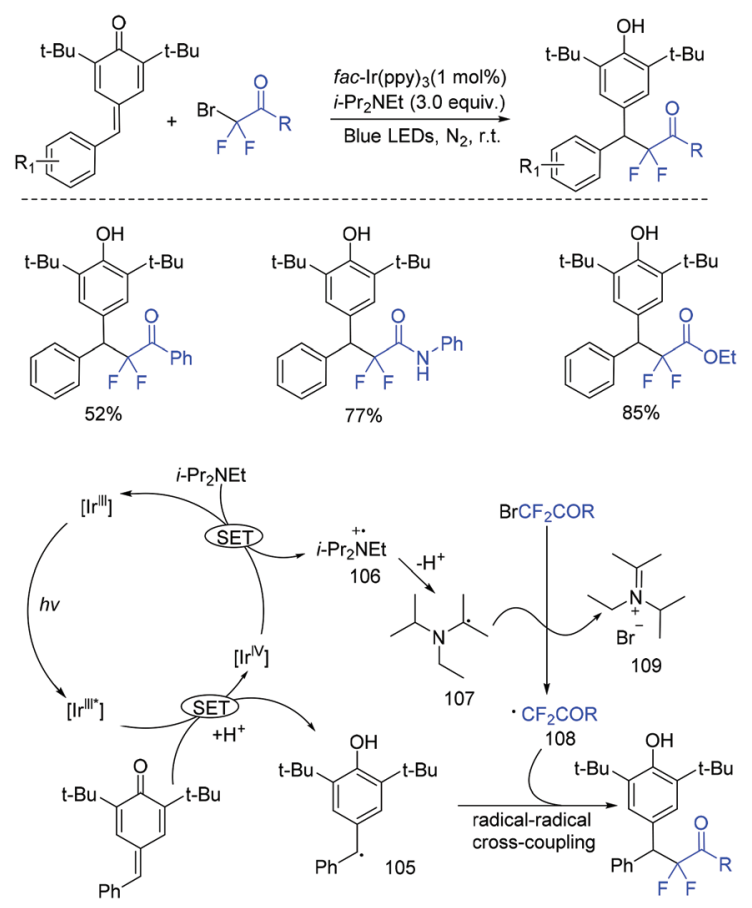

Scheme 55 The visible-light-induced difluoroalkylation reaction of $p$-QMs.

$\mathrm{BrCF}_{2} \mathrm{CO}_{2} \mathrm{Et}$ to produce $\operatorname{Ir}(\mathrm{III})$ and $\mathrm{CF}_{2} \mathrm{CO}_{2} \mathrm{Et}$ radical respectively.

Muñoz-Molina and Perez found that $\left[\mathrm{Cp}{ }^{*} \mathrm{RuCl}\left(\mathrm{PPh}_{3}\right)_{2}\right]$ complex showed good catalytic activity for the Heck-type alkenylation of alkyl bromides (Scheme 56). ${ }^{128}$ Tertiary, secondary, and primary alkyl bromides could all react smoothly in this reaction. The corresponding product could be obtained in 91\% yield when $\mathrm{BrCF}_{2} \mathrm{CO}_{2}$ Et was used. Hünig's base played an important role for this transformation. Preliminary mechanistic studies support the participation of alkyl radicals in the reaction. The mechanism is outlined in Scheme 56. Initially, the formation of carbon-centered radicals by a SET process was induced by the half-sandwich ruthenium(II) complex, which was followed by the anti-Markovnikov addition with the

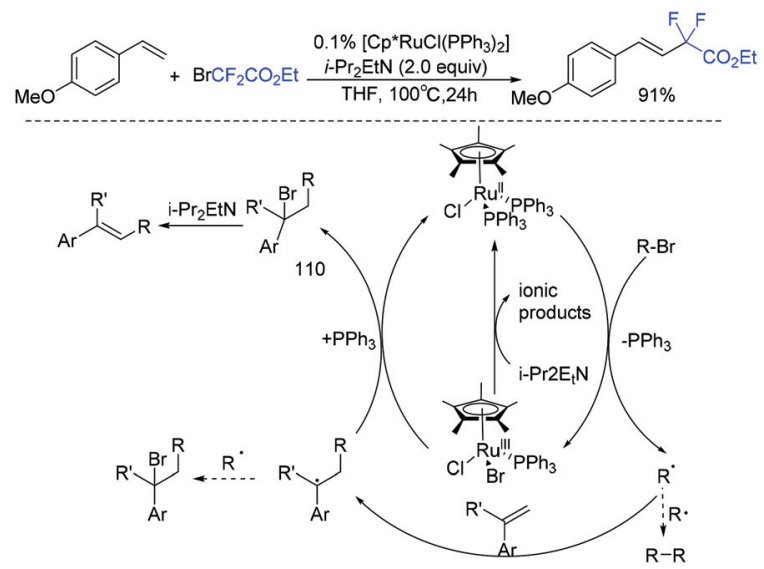

Scheme 56 The Ru complex-catalyzed Heck-type alkenylation.

olefin to deliver another radical species. Then the new radical species reduced the ruthenium(III) center to ruthenium(II), with the release of intermediate 110. Finally, the desired product was obtained via base-induced elimination reaction. Additionally, the amine acts as a reductant to regenerate the $\mathrm{Ru}(\mathrm{II})$ catalyst from $\mathrm{Ru}(\mathrm{III})$.

The visible-light-induced coupling of difluoromethyl halides with allyl sulfones for the synthesis of 4,4-difluoroalkenes was reported by the Ryu group (Scheme 57). ${ }^{129}$ With the help of catalytic $\mathrm{Ru}(\mathrm{bpy})_{3} \mathrm{Cl}_{2}$, diisopropylethylamine, and Hantzsch ester, the reaction proceed effectively within $2 \mathrm{~h}$. In the catalytic cycle, $\mathrm{Ru}(\mathrm{I})$ was reduced by $\mathrm{HEH}$ and/or amine from the photoexcited $\mathrm{Ru}(\mathrm{II})^{*}$ via a SET process. Then another SET process occurred between $\mathrm{Ru}(\mathrm{I})$ and $\mathrm{BrCF}_{2} \mathrm{CO}_{2} \mathrm{Et}$ to generate $\mathrm{CF}_{2} \mathrm{CO}_{2} \mathrm{Et}$ radical, which was followed by an addition reaction with $\mathrm{C}$ and $\beta$-fragmentation to furnish the product. In 2020, the Yao group found that the decarboxylative allylation of $\alpha, \alpha$-difluoroarylacetic acids with allyl sulfones can also be catalyzed by silver in water. ${ }^{130}$ Preliminary mechanistic investigations suggest that a radical process and $\beta$-elimination might be involved.

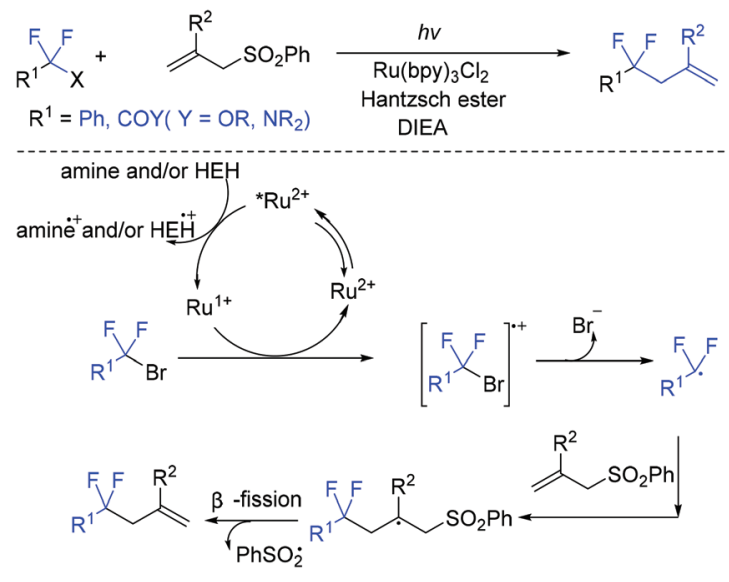

Scheme 57 The visible-light-induced coupling of difluoromethyl halides with allyl sulfones. 


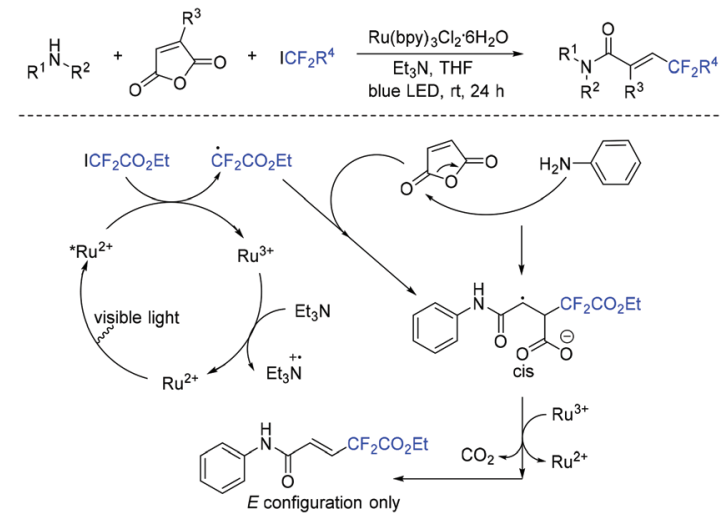

Scheme 58 The photoinduced decarboxylative difunctionalization of maleic anhydride with amine and fluorinated alkyl iodides.

The photoinduced decarboxylative difunctionalization of maleic anhydride with amine and fluorinated alkyl iodides was reported by the Zhang group (Scheme 58). ${ }^{131}$ A variety of fluorinated alkyl halides and amines reacted smoothly in this reaction. The difluoroalkyl radical which was generated from oxidative quenching reaction between fluorinated alkyl iodides and excited $\mathrm{Ru}(\mathrm{II})^{*}$ was also possible in this reaction.

Coumarins are an important class of heterocyclic compounds which display a wide range of biological activities. $^{132-135}$ In 2019, the same group found that difluoroalkylation of ortho-hydroxycinnamic esters with $\mathrm{BrCF}_{2} \mathrm{CO}_{2} \mathrm{Et}$ can proceed smoothly through a visible-light-induced cascade radical cyclization process (Scheme 59). A variety of 3-fluoroalkylated coumarins were successfully prepared under mild conditions. Similarly, $\mathrm{RCF}_{2}$ radical species was also proposed in the reaction cycle. ${ }^{136}$

\section{Difluoroalkylation with alkynes}

\subsection{Carbodifluoroalkylation with alkynes}

In 2015, the Fu group reported the visible-light-promoted aryldifluoroacetylation of alkynes with ethyl bromodifluoroacetate for the synthesis of 3-difluoroacetylated coumarins (Scheme 60). ${ }^{40}$ A wide range of functional groups was tolerated in this reaction. A tandem radical cyclization process which involved $\mathrm{RCF}_{2}$ radical was proposed for this reaction.

A mild and efficient photocatalytic protocol for the synthesis of difluoroacetylindenones via the reaction of 1,3-diarylpropynones with ethyl bromodifluoroacetate was established by Rastogi and coworkers (Scheme 61). ${ }^{137}$ The visible-light-catalyzed difluoroacetylation of 1,3-diarylpropynones followed by intramolecular radical cyclization was proposed for this reaction.

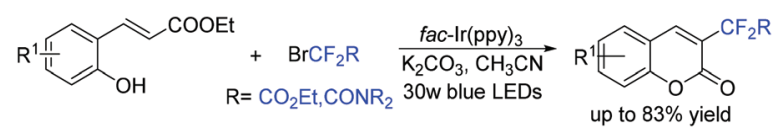

Scheme 59 The difluoroalkylation of ortho-hydroxycinnamic esters.

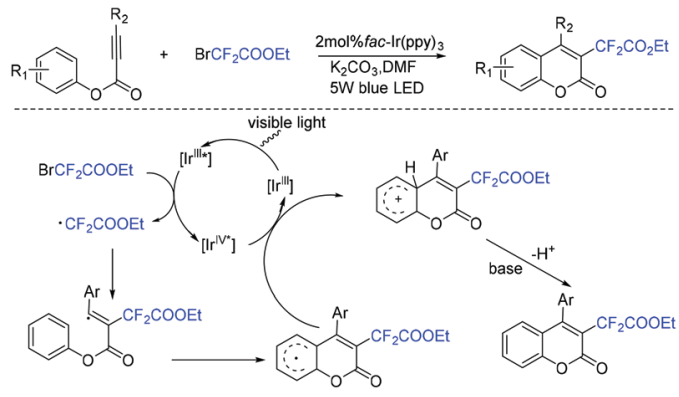

Scheme 60 The visible-light-promoted aryldifluoroacetylation of alkynes with ethyl bromodifluoroacetate.

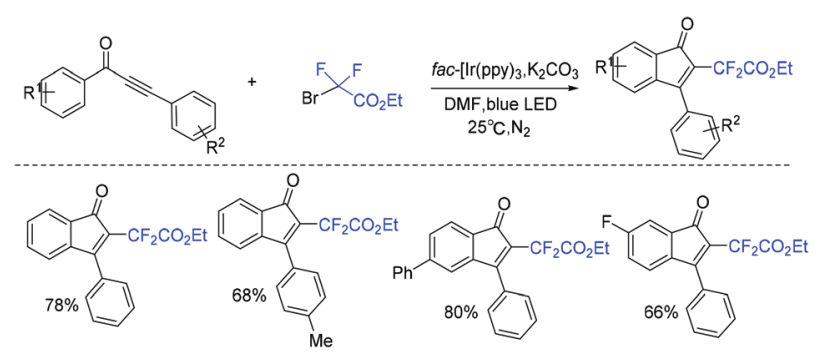

Scheme 61 The synthesis of difluoroacetylindenones via the reaction of 1,3-diarylpropynones with ethyl bromodifluoroacetate.

In the presence of $\operatorname{Ir}(\text { ppy })_{3}$ as photocatalyst, the group of $\mathrm{Li}$ and $\mathrm{Hu}$ found that $\mathrm{N}$-benzyl- $\mathrm{N}$-(2-ethynylaryl)amides were good partners to react with ethyl bromodifluoroacetate for the synthesis of substituted dibenzazepines (Scheme 62). ${ }^{138}$ Two carbon-centered functional groups are introduced across the $\mathrm{C} \equiv \mathrm{C}$ bond in a single reaction. The $\mathrm{Xu}$ group found that this reaction can also smoothly proceed in the presence of organic photocatalysts (cat-PMP) (Scheme 63). ${ }^{139}$ Fluoroalkyl radical which formed from the reduction of $\mathrm{BrCF}_{2} \mathrm{CO}_{2} \mathrm{Et}$ by excitedstate cat-PMP* was proposed in the catalytic cycle. Then intermolecular addition of fluoroalkyl radical $\mathrm{RCF}_{2}$ to $\mathrm{N}$-benzyl- $\mathrm{N}$ (2-ethynylaryl)amides occurred to provide radical 112, which was followed by intramolecular addition to form intermediate 114. Intermediate 114 was oxidized by cat-PMP ${ }^{++}$to afford $\mathbf{1 1 5}$. Finally, intermediate $\mathbf{1 1 5}$ undergoes deprotonation to furnish the desired product. On the other hand, another possible pathway ( path b) was also possible for this reaction.

Copper-catalyzed cascade radical addition/dearomative spirocyclization of biaryl ynones to access difluoromethylated spiro $[5.5]$ trienones was first realized by the Zhu group (Scheme 64). ${ }^{140}$

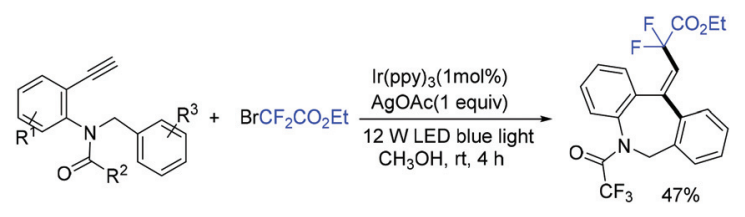

Scheme 62 The synthesis of substituted dibenzazepines catalyzed by $\mathrm{Ir}$ $(\text { ppy })_{3}$. 

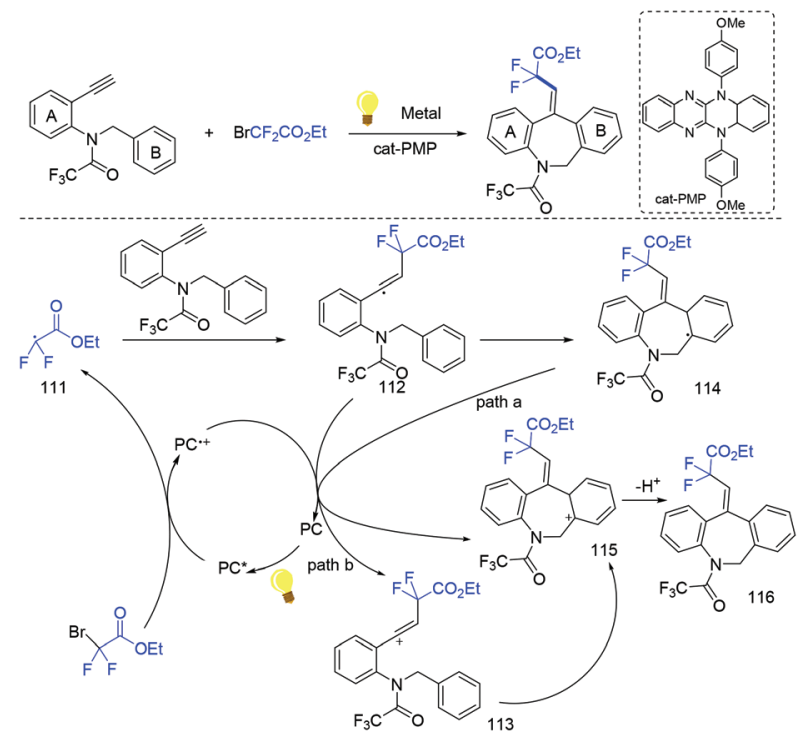

Scheme 63 The synthesis of substituted dibenzazepines catalyzed by organic photocatalysts.

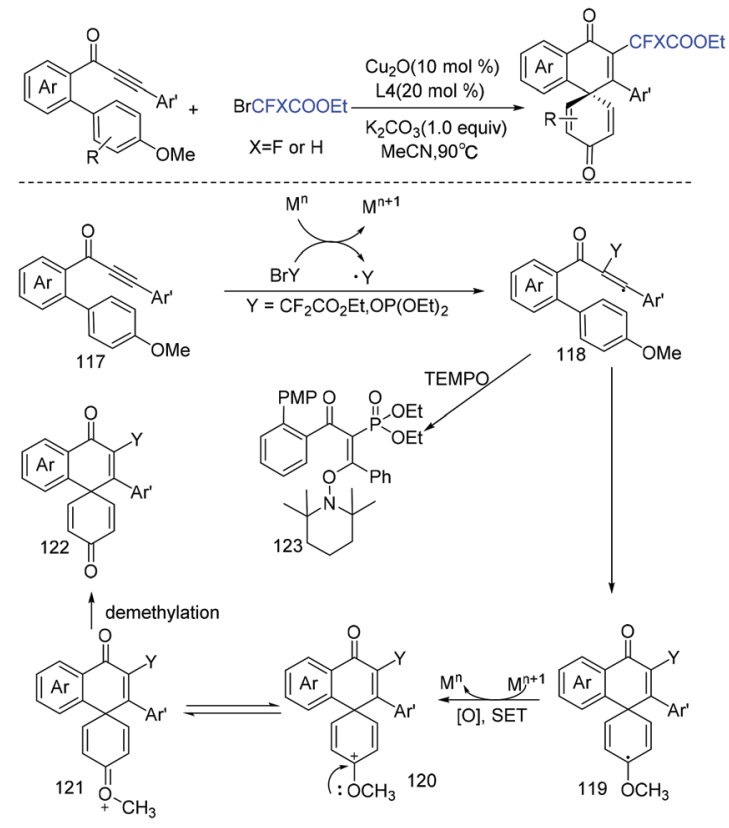

Scheme 64 The copper-catalyzed cascade radical addition/dearomative spirocyclization of biaryl ynones.

This reaction is useful for the construction of spiro compounds. When $\mathrm{Ag}$ was employed as catalyst, monofluoromethylated and phosphorated spiro[5.5]trienones were also obtained using diethylphosphite as substrate. Fluoroalkyl radical was proposed in the reaction mechanism. A vinyl radical $\mathbf{1 1 7}$ was formed via the addition of $\mathrm{RCF}_{2}$ radical to $\mathrm{C}-\mathrm{C}$ triple bond, then 6-exo-trig cyclization occurred to afford intermediate $\mathbf{1 1 9}$ which is oxidized by $\mathrm{M}^{n+1}$ to form oxocarbenium ion 120. After demethylation, the desired product was obtained.

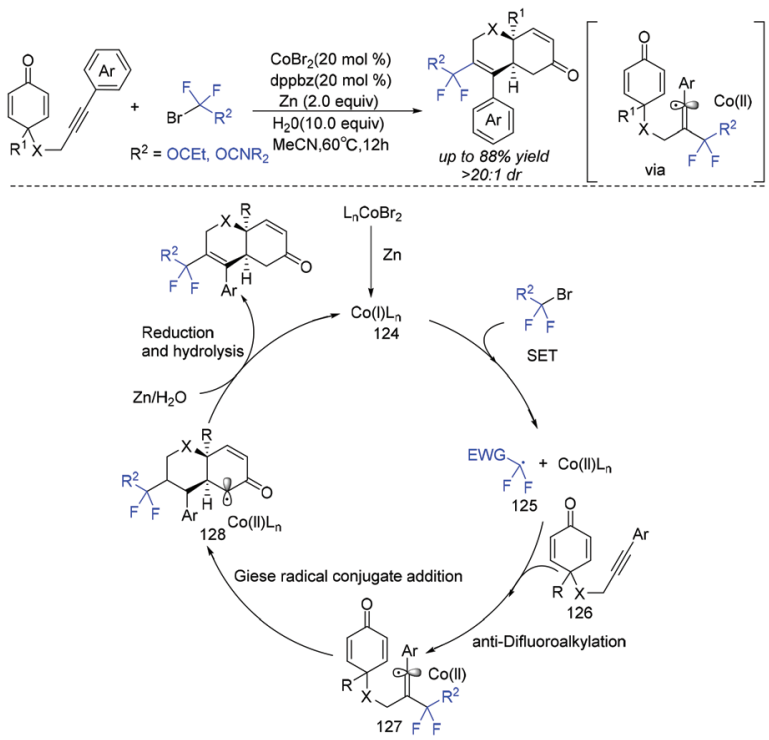

Scheme 65 The cobalt-promoted cyclization of fluoroalkyl radical with C-C triple bond.

In 2019, the group of Ackermann and Li demonstrated that cobalt was a good catalyst to promote the cyclization of fluoroalkyl radical with $\mathrm{C}-\mathrm{C}$ triple bond (Scheme 65). ${ }^{141}$ Various fluorinated chromenes were synthesized via cobalt-catalyzed difluoroalkylation/Giese radical conjugate cyclization manifold with various alkyne-tethered cyclohexadienones and halogenated fluorinating reagents. Similar to copper catalyst, the Co catalyst could also reduce ethyl bromodifluoroacetate to produce difluoroalkyl radical. Subsequently, the addition of difluoroalkyl radical with alkynes resulted in alkenyl radical 127. Then intermediate 127 undergoes an intramolecular Giese radical conjugate addition to afford the key intermediate 128, which was followed by reduction by $\mathrm{Zn}$, along with hydrolysis, providing the desired product, with the release of the active $\operatorname{Co}(\mathrm{I}) \mathrm{Ln}$ complex.

Cyclopropenes having unique rigid structure and variable reactivity have attracted much interest. ${ }^{142-145}$ In 2019, the Ma group $^{146}$ reported the first highly enantioselective $\mathrm{Rh}^{\mathrm{II}}$-catalyzed cyclopropenation reaction of unactivated internal alkynes with a difluorodiazoethane reagent (PsDFA) (Scheme 66). A broad range of enantioenriched unsaturated three-membered carbocycles can be obtained smoothly. Subsequently, various reactions such as crosscouplings, hydrogenation, Diels-Alder reaction, and Pauson-Khand reaction could occur to demonstrate the synthetic utility of the strained carbocycles.

$$
\mathrm{Ar}=\mathrm{R}+\underset{\mathrm{CF}_{2} \mathrm{SO}_{2} \mathrm{Ph}}{\stackrel{\mathrm{Rh}_{2}(\mathrm{~S}-\mathrm{TCPTTL})_{4}}{\mathrm{CH}_{2} \mathrm{Cl}_{2}, 40 \mathrm{~min}}} \underset{\text { up to } 99 \% \text { yield and } 96 \% \text { ee }}{\stackrel{\mathrm{N}_{2}}{\longrightarrow}} \overbrace{\mathrm{R}}^{\mathrm{C}_{2} \mathrm{SO}_{2} \mathrm{Ph}}
$$

Scheme 66 The palladium-catalyzed difluoroalkylation reaction of $N$-propargylamides. 


\subsection{Hydrodifluoroalkylation with alkynes}

The group of Wang and Zhao found that the hydrodifluoroalkylation of alkynes with ethyl bromodifluoroacetate could proceeded smoothly mediated by copper. ${ }^{147}$ Metabisulfite as reduction agent should be used for this reaction to suppress the homocoupling of terminal alkynes. It can be seen from Scheme 67 that $\mathrm{CF}_{2} \mathrm{COOEt}$ radical was generated from the reaction of $\mathrm{BrCF}_{2} \mathrm{COOEt}$ and $\mathrm{Cu}(\mathrm{I})$ via SET process. Then addition reaction with (phenylethynyl)copper 130, hydrogen abstraction from solvents and protonation by moisture occurred in sequence to give the final product.

\subsection{Heterodifluoroalkylation with alkynes}

A facile method for the synthesis of C2-tetrasubstituted indolin-3-ones via copper-catalyzed redox cycloisomerization of nonprefunctionalized nitroalkynes with $\mathrm{BrCF}_{2} \mathrm{CO}_{2} \mathrm{Et}$ was achieved by the Song group (Scheme 68). ${ }^{148}$ Using diboron as the reductant, this reaction proceeded in a one-pot protocol forming a fluorine-containing non-carbon quaternary center. One of the oxygens from $\mathrm{NO}_{2}$ was captured by $\mathrm{B}_{2} \mathrm{pin}_{2}$ with the aid of $\mathrm{Na}_{2} \mathrm{CO}_{3}$. Another oxygen in $\mathrm{NO}_{2}$ was transformed into $\mathrm{C}=\mathrm{O}$ bond of $\mathrm{C} 2$-tetrasubstituted indolin-3-ones.

In 2019, the same group developed a visible-light-promoted cyclization of methylthiolated alkynones with $\mathrm{BrCF}_{2} \mathrm{CO}_{2} \mathrm{Et}$ for the synthesis of 2-substituted thiochromones. ${ }^{149}$ The desired products could be accessed with moderate to good yields via $\mathrm{C}(\mathrm{sp} 2)-\mathrm{S}$ bond formation and $\mathrm{C}(\mathrm{sp} 3)-\mathrm{S}$ cleavage. Besides $\mathrm{CF}_{2} \mathrm{CO}_{2}$ Et group, phosphoryl, sulfenyl, and acyl groups are also well tolerated in this method. The reaction mechanism is depicted in Scheme 69. Firstly, the fluoroalkyl radical was generated from the corresponding conditions. Then the $\mathrm{C}-\mathrm{C}$ triple bond in alkynones was attacked by fluoroalkyl radical to give vinyl radical 133. Finally, 6-exo-trig cyclization of 133 with the

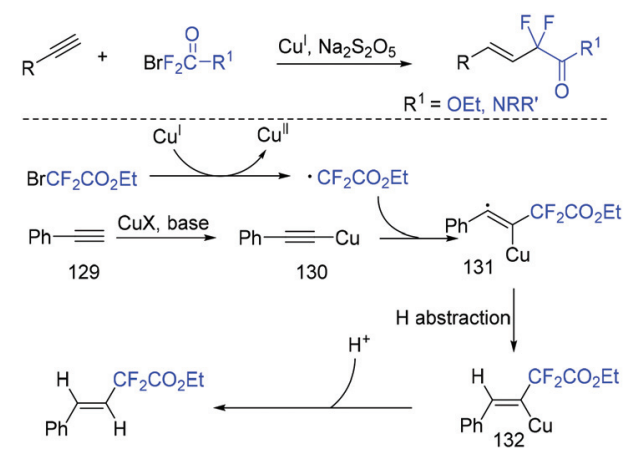

Scheme 67 The copper-catalyzed hydrodifluoroalkylation of alkynes with ethyl bromodifluoroacetate.

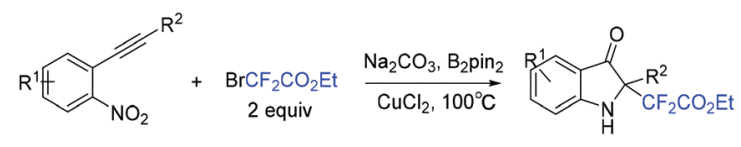

Scheme 68 The synthesis of C2-tetrasubstituted indolin-3-ones.

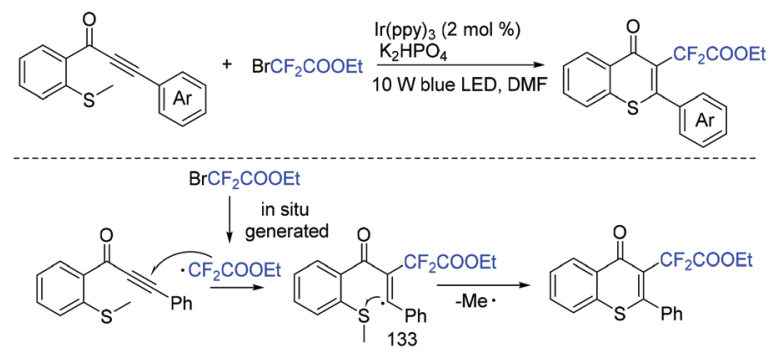

Scheme 69 The cyclization of methylthiolated alkynones with $\mathrm{BrCF}_{2} \mathrm{CO}_{2} \mathrm{Et}$ for the synthesis of 2-substituted thiochromones.

SMe moiety gave the desired product along with the release of methyl radical.

The carbon radical intermediates generated from the electrophilic radical addition of $\mathrm{CF}_{2} \mathrm{CO}_{2}$ Et radical with alkynes can be trapped by an oxygen nucleophile to form difluoro-substituted heterocycles. ${ }^{40,150,151}$ In 2018, a palladium-catalyzed difluoroalkylation reaction of $N$-propargylamides with ethyl iododifluoroacetate for the synthesis of difluoromethyl oxazoles and oxazolines was reported by the Liang group (Scheme 70). ${ }^{152} \mathrm{~A} \operatorname{Pd}(0) / \operatorname{Pd}(\mathrm{I}) / \mathrm{Pd}(\mathrm{II})$ cycle was proposed for the reaction mechanism. Additionally, perfluoroalkyl iodides were also suitable for the generation of perfluoroalkyl heterocycles.

An efficient cobalt-catalyzed difluoroalkylation of alkynes/ alkenes for the construction of fluorinated hydrocarbons was described by Jacobi von Wangelin and coworkers. ${ }^{153}$ However, alkyl-substituted terminal alkynes were not effective in this system. This was the first cobalt-catalyzed reaction with high stereoselectivity. The proposed reaction mechanism is shown

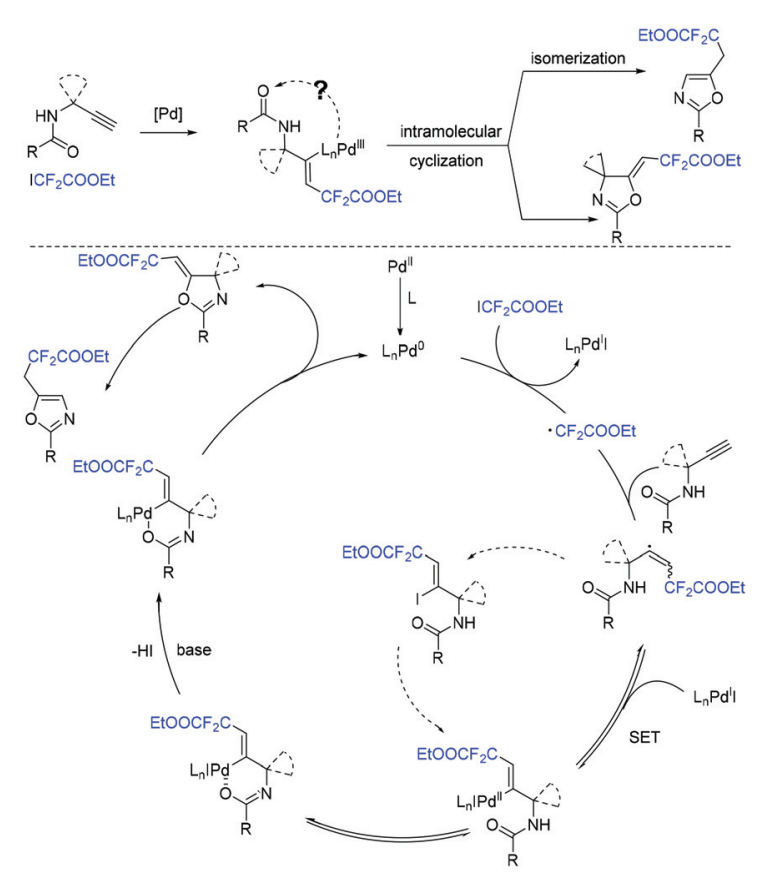

Scheme 70 The palladium-catalyzed difluoroalkylation reaction of $N$-propargylamides. 


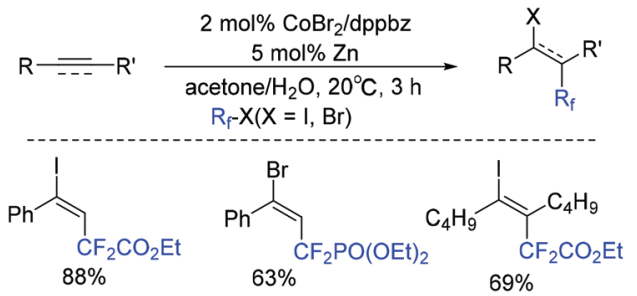

Scheme 71 The cobalt-catalyzed difluoroalkylations of alkynes/ alkenes.

in Scheme 71. Different from related cobalt-catalyzed reductive couplings and Heck-type reactions, terminal halogen atom transfer mediated by Co catalyst was involved in the radical chain mechanism, which avoided the use of a stoichiometric sacrificial reductant. The $\mathrm{R}_{\mathrm{F}} \mathrm{Co}^{\mathrm{III}} \mathrm{X}$ complex which delivered the free $\mathrm{RF}$ radical and mediated the halogen atom transfer to the terminal vinyl radical played an important role in the catalytic cycle.

In 2018, a palladium-mediated three-component reaction of alkynes with fluoroalkyl halides and diboron reagents was developed by the group of Zhu and Bai (Scheme 72). ${ }^{154 a}$ Both internal and terminal alkynes were tolerated in this reaction, providing the functionalized 1,2-fluoroalkylboronated alkenes with excellent regio- and stereoselectivity. In the catalytic cycle, a SET process was also proposed between fluoroalkyl halides and $\operatorname{Pd}(0)$ to afford $\mathrm{RCF}_{2}$ radical and $\operatorname{Pd}(\mathrm{I})$ species. Then the addition reaction of $\mathrm{RCF}_{2}$ radical and alkynes took place to give vinyl radical. Then oxidative addition and base-assisted transmetalation with $\mathrm{B}_{2}$ pin $_{2}$ delivering the alkenyl(boryl)palladium intermediate 137 and reductive elimination of 137 occurred successively to give the desired product (path a). Alternatively, path $\mathrm{b}$ featuring the direct reaction of vinyl radical I with $\operatorname{Pd}(\mathrm{I})$ was also possible. But this pathway was less likely. The palladium-catalyzed trans-fluoroalkylation-borylation of alkynes with fluoroalkyl iodides and $\mathrm{B}_{2} \mathrm{pin}_{2}$ was also realized by the Zhang group. ${ }^{154 b}$

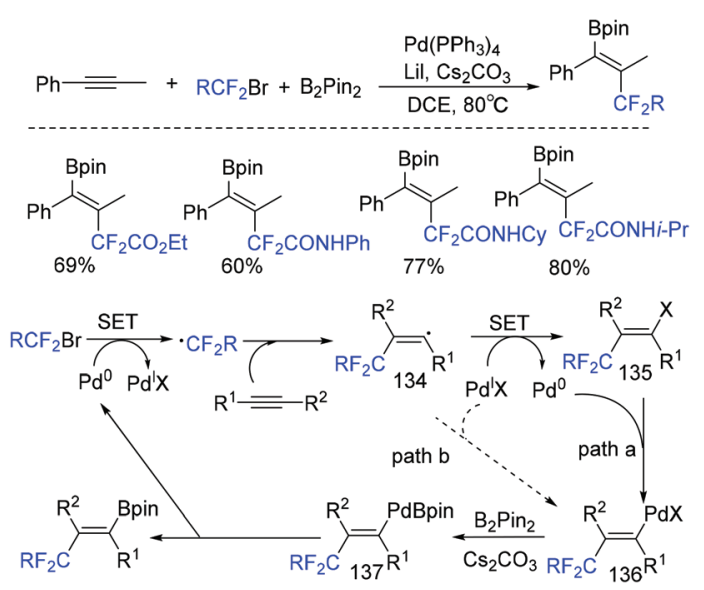

Scheme 72 The palladium-mediated three-component reaction of alkynes with fluoroalkyl halides and diboron reagents.

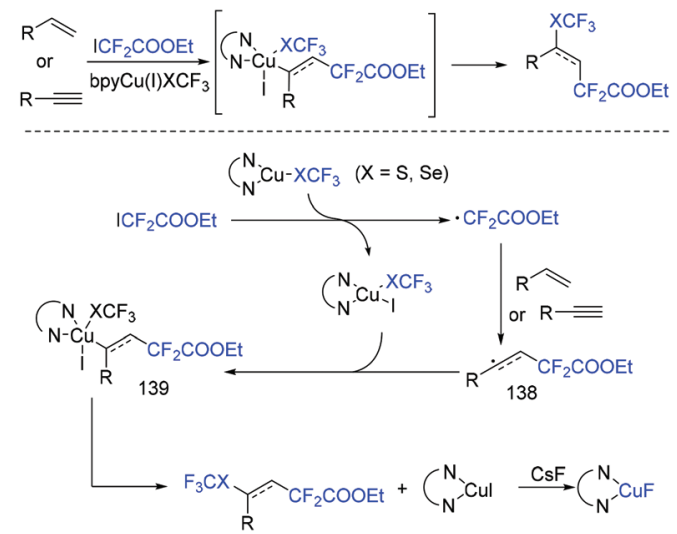

Scheme 73 The copper-catalyzed difluoroalkylation reaction of alkynes with ethyl iododifluoroacetate.

In 2018, the Liang group found that air-stable $\mathrm{SCF}_{3}$ and $\mathrm{SeCF}_{3}$ reagents could act as free-radical initiators of ethyl iododifluoroacetate via reduction reaction (Scheme 73). ${ }^{155}$ In the presence of air-stable $\mathrm{SCF}_{3}$ and $\mathrm{SeCF}_{3}$ reagents, the difluoroalkylation reaction of alkynes with ethyl iododifluoroacetate proceeded smoothly. $\beta$-Proton elimination was successfully avoided. Besides alkynes, alkenes were also suitable in this reaction. $\mathrm{A} \mathrm{Cu}(\mathrm{I})-\mathrm{Cu}(\mathrm{II})-\mathrm{Cu}(\mathrm{III})$ mechanism was described for this reaction.

A photocatalyst-free fluoroalkylation reaction of alkynes with fluoroalkyl iodides was developed by the He group (Scheme 74). ${ }^{156}$ It is worth noting that the noncovalent interactions between acetone and fluoroalkyl iodides could assist the formation of fluoroalkyl radical. Additionally, (hetero) arenes and alkenes were also tolerated in this transformation.

\subsection{Other difluoroalkylation with alkynes}

In 2019, the visible-light-induced alkynyl-difluoroalkylation of alkynyl halides with fluoroalkyl halides to access a diverse range of fluoroalkylalkynes was described by the Cho group (Scheme 75). ${ }^{157}$ Controlled experiments suggested a radical mechanism was possible. SET from $f a c$-Ir $(\mathrm{ppy})_{3}{ }^{*}$ to fluoroalkyl

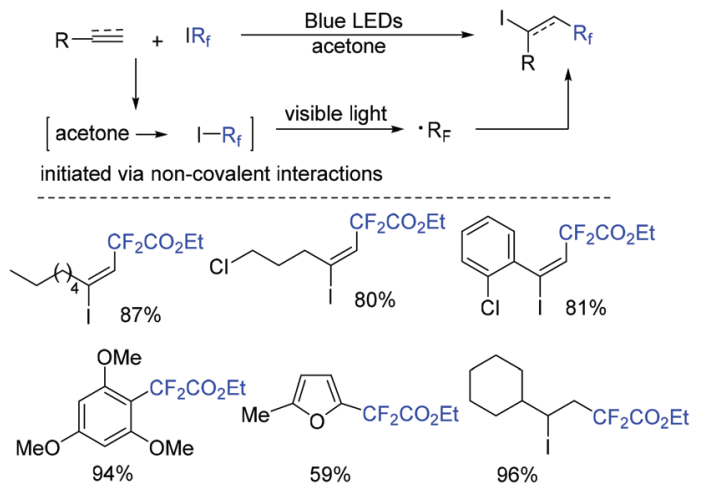

Scheme 74 The photocatalyst-free fluoroalkylation reaction of alkynes with fluoroalkyl iodides. 

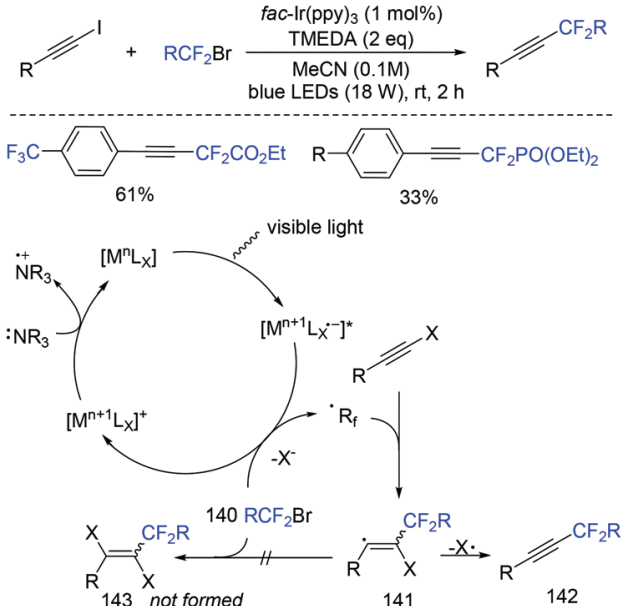

Scheme 75 The visible-light-induced alkynyl-difluoroalkylation of alkynyl halides with fluoroalkyl halides.

halide generated the oxidized photocatalyst $f a c-\operatorname{Ir}(\mathrm{ppy})_{4}$ and the $\mathrm{RF}_{2}$ radical. The fac-Ir(ppy $)_{4}$ could be reduced by TMEDA to form $f a c-\operatorname{Ir}(\mathrm{ppy})_{3}$ complex. Then vinyl radical $\mathbf{1 4 1}$ was formed via the addition reaction of $\mathrm{RCF}_{2}$ radical and alkynyl halide, which could be converted to the final product via dehalogenation.

In 2019, a palladium-catalyzed ligand-free difluoroalkylation of alkynes with iododifluoroacetones for the construction of difluorinated 1,3-enynes was developed by the $\mathrm{Wu}$ group (Scheme 76). ${ }^{158}$ The radical addition of $\mathrm{RCOCF}_{2}$ radical which was generated from reduction of iododifluoroacetones with $\operatorname{Pd}\left(\mathrm{PPh}_{3}\right)_{4}$ to alkynes and subsequent Sonogashira coupling with the same alkynes were involved in the reaction mechanism.

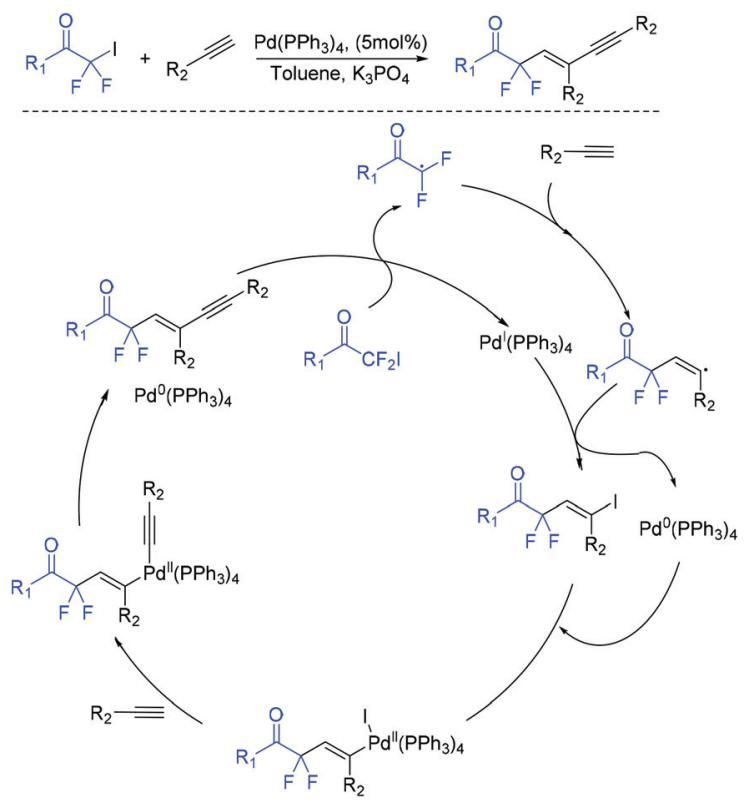

Scheme 76 The construction of difluorinated 1,3-enynes.

\section{Difluoroalkylation with arene}

In 2018, an efficient para-selective $\mathrm{C}-\mathrm{H}$ difluoromethylation of aromatic ketones catalyzed by tetrakis(triphenylphosphine)palladium(0) was developed by the Zhao group (Scheme 77). ${ }^{159}$ Additionally, several well-known drugs such as oxybenzone, ketoprofen, zaltoprofen, and propafenone were also well tolerated in this system. The role of AgF was unclear, it being likely to assist the palladium complex in activating the aromatic ring. A mechanistic study suggested that a highly para-selective difluoromethylation reaction may result from the palladium complex coordinated to the carbonyl group. SET between $\operatorname{Pd}(\mathrm{I})$ and $\mathrm{BrCF}_{2} \mathrm{CO}_{2} \mathrm{Et}$ took place to afford $\mathrm{CF}_{2} \mathrm{CO}_{2} \mathrm{Et}$ radical and $\mathrm{Pd}(\mathrm{II})$. Then $\mathrm{CF}_{2} \mathrm{COOEt}$ radical coupled with complex $\mathbf{1 4 4}$ which came from the coordination of $\mathrm{Pd}(\mathrm{II})$ intermediate with aromatic ketone to give intermediate $\mathbf{1 4 5}$. Subsequently, intermediate $\mathbf{1 4 5}$ was converted to the final product via SET process and aromatization, with the liberation of $\mathrm{Pd}(\mathrm{I})$ catalyst. Almost at the same time, similar work was described by the group of $\mathrm{Xu}$ and Lou (Scheme 78). ${ }^{160}$

In 2019, Li and coworkers found that para-selective $\mathrm{C}-\mathrm{H}$ difluoroalkylation of aromatic carbonyls could also be induced by visible light. ${ }^{161}$ In the presence of $\operatorname{Ir}(\mathrm{ppy})_{3}$ as the catalyst and 1,10-phenanthroline as the additive, various products could be obtained efficiently. The proposed mechanism is
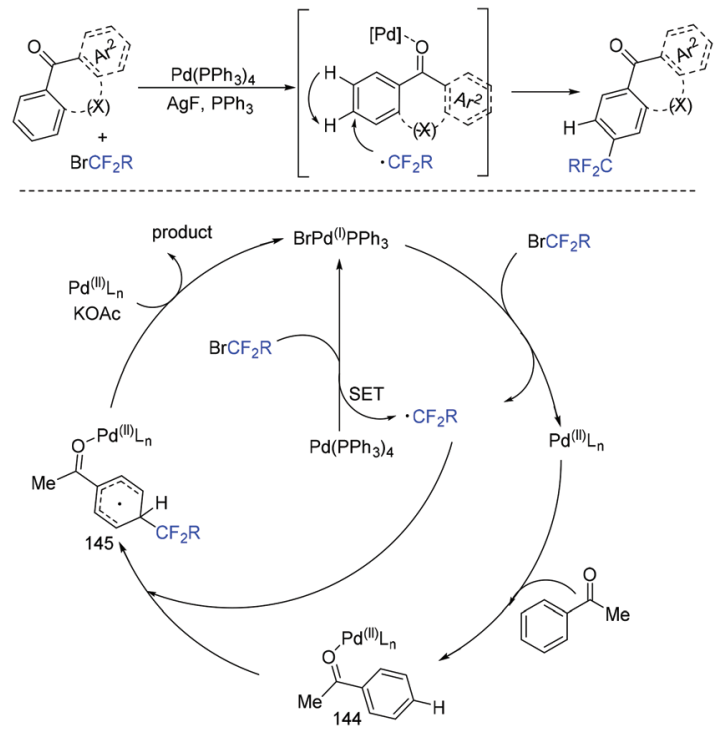

Scheme 77 The para-selective $\mathrm{C}-\mathrm{H}$ difluoromethylation of aromatic ketones.

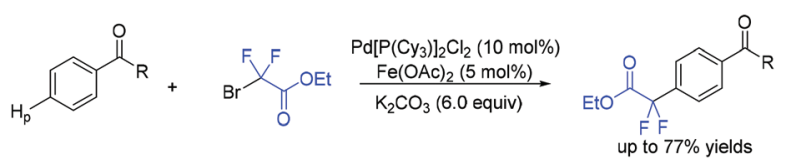

Scheme 78 The para-selective $\mathrm{C}-\mathrm{H}$ difluoromethylation of aromatic ketones. 

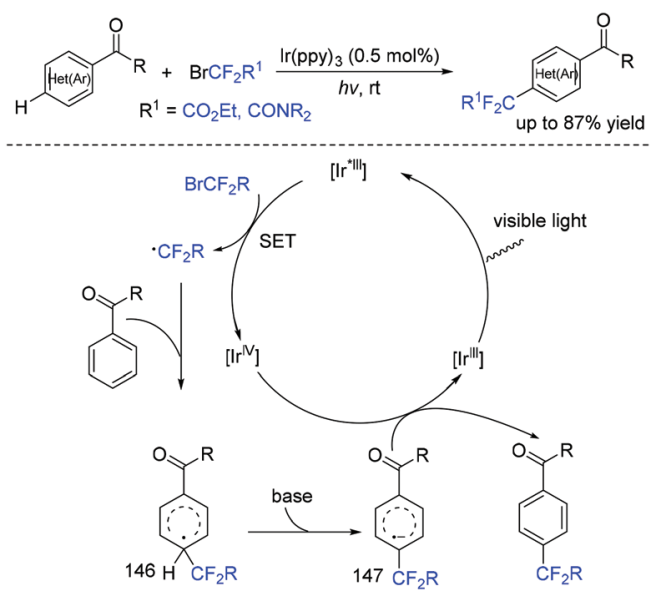

Scheme 79 The para-selective $\mathrm{C}-\mathrm{H}$ difluoromethylation of aromatic ketones.

shown in Scheme 79. Concurrently, irradiation of $\operatorname{Ir}^{\mathrm{III}}(\mathrm{ppy})_{3}$ resulted in excited $\operatorname{Ir}^{\mathrm{III}}(\mathrm{ppy})_{3}{ }^{*}$, which could be oxidized by $\mathrm{BrCF}_{2} \mathrm{CO}_{2} \mathrm{Et}$ via a SET process with the formation of $\mathrm{CF}_{2} \mathrm{CO}_{2} \mathrm{Et}$ radical and $\operatorname{Ir}^{\mathrm{IV}}(\mathrm{ppy})_{3}$. Then the para-selective addition of $\mathrm{CF}_{2} \mathrm{CO}_{2} \mathrm{Et}$ radical to aryl carbonyls occurred to produce intermediate 146, which could be converted to radical anion intermediate 147 by the reverse process of deprotonation. Finally, intermediate 147 was oxidized by $\operatorname{Ir}^{\mathrm{IV}}$ to give the product via rearomatization. In the same year, similar reactions of visiblelight-induced difluoroalkylation of aromatic aldehydes were developed by the Xu group (Scheme 80). ${ }^{162}$

Using ruthenium(II) as a catalyst, para-selective $\mathrm{C}-\mathrm{H}$ difluoromethylation of ketoxime ethers was also realized by the Zhao group, providing the corresponding para-difluoromethylated products in moderate to good yield (Scheme 81). ${ }^{163}$ Mechanistic studies showed that chelation-assisted cycloruthenation may play an important role in the para selectivity of the difluoromethylation of ketoxime ethers. Different from Zhao's work, meta-C-H alkylation of arenes catalyzed by $\mathrm{Ru}$ was described by Ackermann in the presence of visible light (Scheme 82a). ${ }^{164}$ Both tertiary and secondary alkyl bromides were compatible with this reaction. When $\mathrm{BrCF}_{2} \mathrm{CO}_{2} \mathrm{R}$ was employed, the product was isolated in $48 \%$ yield. Recently, the Ru-catalyzed meta-C-H bond difluoroalkylation of 2-phenoxypyridines was realized by the Cui group (Scheme 82b). ${ }^{165}$

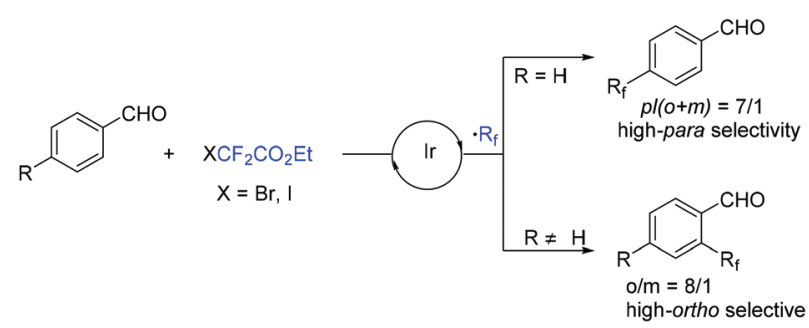

Scheme 80 The para-selective $\mathrm{C}-\mathrm{H}$ difluoromethylation of aromatic aldehydes.

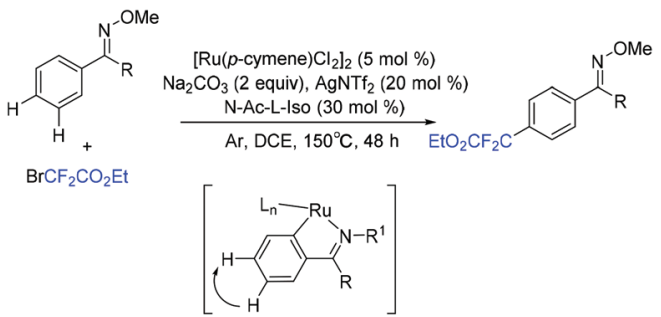

Scheme 81 The para-selective $\mathrm{C}-\mathrm{H}$ difluoromethylation of ketoxime ethers.
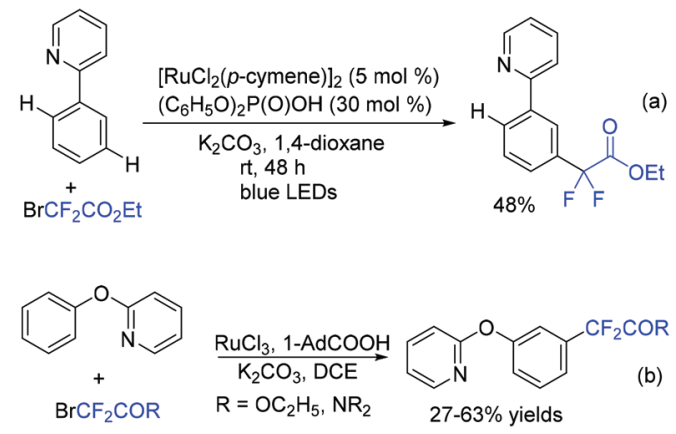

Scheme 82 The meta-C-H alkylation of arenes catalyzed by $\mathrm{Ru}$.

The ruthenium(II)-catalyzed para- and meta-selective $\mathrm{C}-\mathrm{H}$ difluoroalkylation of aniline derivatives with $\mathrm{BrCF}_{2} \mathrm{CO}_{2}$ Et was reported by the Liang group (Scheme 83). ${ }^{166}$ Mechanistic investigation implied that the key factor of the para-selective difluoroacetylation derived from the cycloruthenation that occurred at $\mathrm{N}-\mathrm{H}$ and $\mathrm{C}-\mathrm{H}$ simultaneously. The electronic effects of carboxylate ligands along with a base determined the selectivity. This reaction rendered readily the modification of anilines as well as the structural motifs of biologically active compounds. Notably, meta-substituted products were obtained when $\mathrm{ClCF}_{2} \mathrm{CO}_{2}$ Et and $\mathrm{BrCF}_{2} \mathrm{CO}_{2}$ Et were applied to this reaction.

Use of $\mathrm{Cu}$ (Scheme $84 \mathrm{a})^{167}$ and $\mathrm{Ru}$ (Scheme $\left.84 \mathrm{~b}\right)^{168}$ to catalyze difluoroalkylation of heteroarenes, including indoles, with $\mathrm{BrCF}_{2} \mathrm{CO}_{2} \mathrm{R}$ was also realized by several groups. However, a mixture of C-2 and C-3 difluoroalkylation was observed in these methods. Therefore, C-3 substituted indoles were usually applied to achieve selective $\mathrm{C}(2)-\mathrm{H}$ difluoroalkylation. The Shi

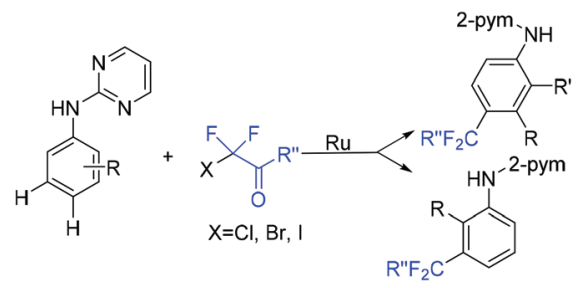

Scheme 83 The ruthenium(॥)-catalyzed para- and meta-selective $\mathrm{C}-\mathrm{H}$ difluoroalkylation of aniline derivatives. 


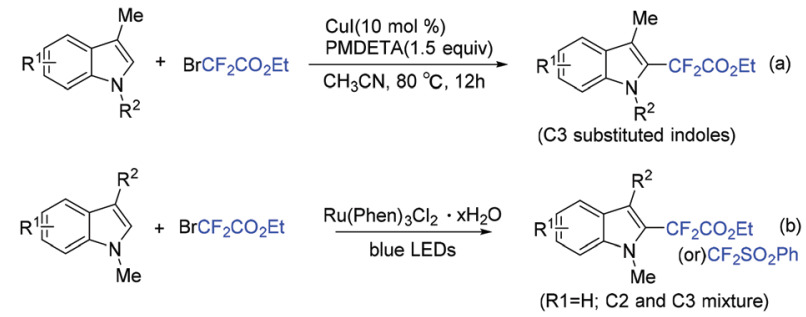

R

Scheme 84 The difluoroalkylation of heteroarenes.

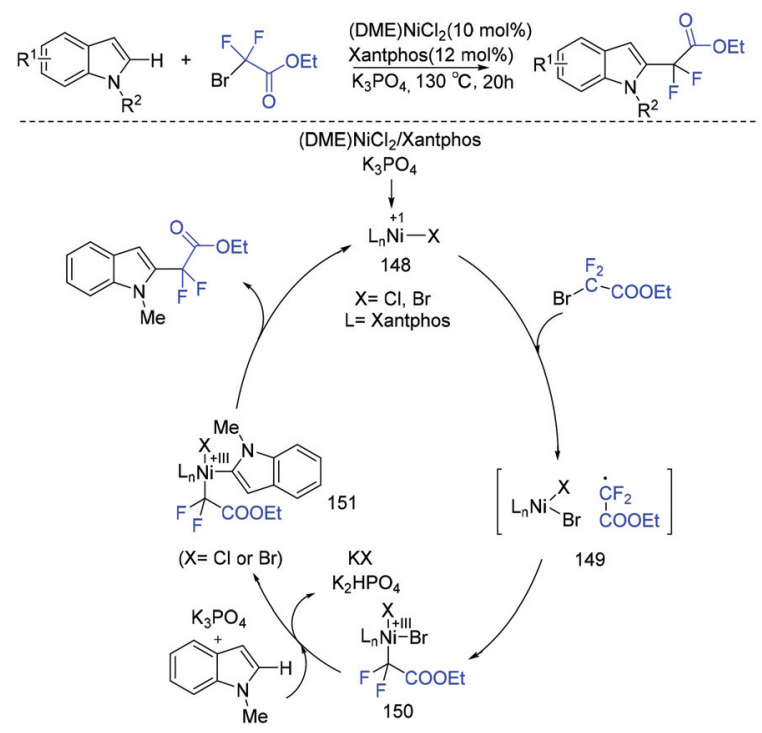

Scheme 85 The nickel-catalyzed $\mathrm{C}(2)-\mathrm{H}$ difluoroalkylation of C-3 unsubstituted indoles.

group realized copper-catalyzed $\mathrm{C}-2$ difluoromethylation of indoles and pyrroles with $\mathrm{BrCF}_{2} \mathrm{CO}_{2}$ Et. But a pyrimidyl group should be present as a directing group (Scheme 84c). ${ }^{169}$ In 2018, the nickel-catalyzed regioselective $\mathrm{C}(2)-\mathrm{H}$ difluoroalkylation of C-3 unsubstituted indoles with $\mathrm{BrCF}_{2} \mathrm{CO}_{2} \mathrm{R}$ was successfully achieved by the Punji group (Scheme 85). ${ }^{170 a}$ Assistance of a directing group at the indoles was not necessary. A possible mechanism is described in Scheme 85. First, the Ni precursor might be reduced by Xantphos and base to active Ni(I) catalyst 148, which was followed by the reaction with $\mathrm{BrCF}_{2} \mathrm{CO}_{2} \mathrm{R}$ to afford intermediate 149. Then intermediate $\mathbf{1 4 9}$ underwent radical rebound to generate $\mathrm{Ni}($ III) species 150, which was converted to intermediate $\mathbf{1 5 1}$ via $\mathrm{C}(2)-\mathrm{H}$ activation of indole. ${ }^{170 b}$ Finally, the product was formed via reductive elimination of 151, with the regeneration of the active catalyst. A parallel $\mathrm{Ni}(0) / \mathrm{Ni}(\mathrm{II})$ path was also possible.

With the aid of DFT calculations, Noël and coworkers synthesized a series of substituted bithiophene derivatives as

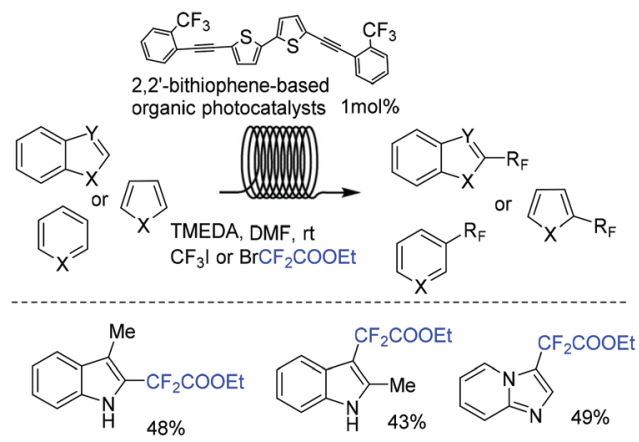

Scheme 86 The visible-light-induced $\mathrm{C}-\mathrm{H}$ functionalization of heteroarenes.

novel and inexpensive organic photocatalysts (Scheme 86). ${ }^{171}$ The activity of these photocatalysts was proved to be comparable with that of other commonly used organic or transitionmetal photocatalysts in $\mathrm{C}-\mathrm{H}$ functionalization of heteroarenes. Additionally, reaction proceeded in continuous-flow photomicroreactor affording the product with moderate to excellent yields within a few minutes. Both reductive and oxidative quenching pathways were proposed in the mechanism.

Imidazopyridines are important structures in numerous bioactive molecules and pharmaceuticals. ${ }^{172,173}$ Difluoroalkylation of imidazopyridines with $\mathrm{BrCF}_{2} \mathrm{CO}_{2}$ Et and a $\mathrm{Cu}$-based catalyst was reported by the Hajra group (Scheme 87a). ${ }^{174}$ In 2017, the Fu group ${ }^{175}$ demonstrated that an Ir photocatalyst was effective for this reaction (Scheme 87b). A variety of desired products with high functional group tolerance were obtained. $\mathrm{CF}_{2} \mathrm{CO}_{2} \mathrm{Et}$ radical derived from the reaction of $\mathrm{BrCF}_{2} \mathrm{CO}_{2} \mathrm{Et}$ and photoexcited state $\left[\mathrm{fac}\right.$-Ir(III)(ppy) $\left.{ }_{3}^{*}\right]$ under light irradiation was proposed for this transformation. Besides imidazopyridines, benzo[d]isoxazoles were also proved to be suitable for Ir-photocatalyzed difluoroalkylation reaction. ${ }^{176}$ The first organophotoredoxcatalyzed difluoromethylenephosphonation of imidazoheterocycles was reported by the Hajra group (Scheme 87c). ${ }^{177}$ Bis (pinacolato)diboron $\left(\mathrm{B}_{2} \mathrm{pin}_{2}\right)$ played an important role for this reaction via activation of imidazoheterocycles. The $\mathrm{Xu}$ group demonstrated that visible-light-induced difluoroalkylation of imidazopyridines could also be realized in the absence of cata-

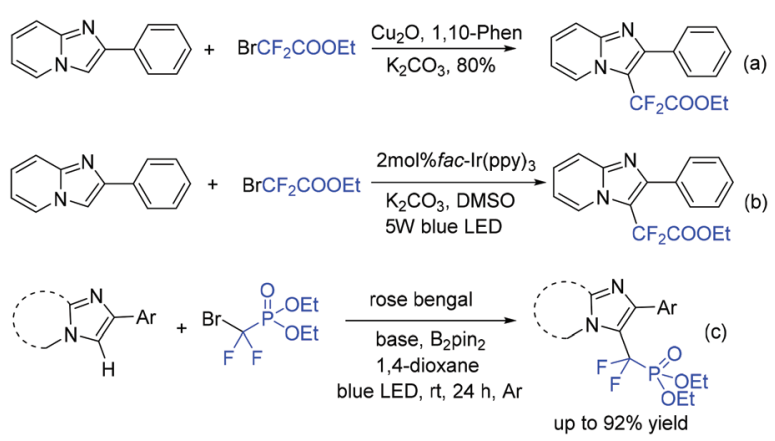

Scheme 87 The difluoroalkylation of imidazopyridines. 


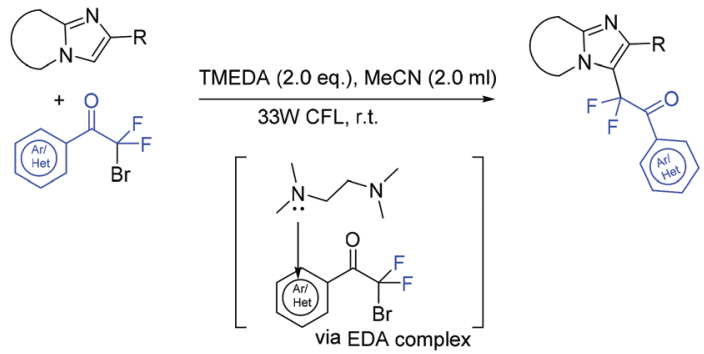

Scheme 88 The visible-light-induced difluoroalkylation of imidazopyridines via EDA.

lyst (Scheme 88). ${ }^{178}$ An energy transfer of bromodifluoroaryl ketones via visible light irradiation affording the excited intermediate bromodifluoroaryl ketones and a rapid $\mathrm{C}-\mathrm{Br}$ bond homolysis to generate the difluoroacyl radical species I via excited intermediate bromodifluoroaryl ketones were proposed for this reaction.

8-Aminoquinoline was also proved to be a good substrate for difluoroalkylation with difluoromethyl bromide reagent. For example, the $\mathrm{Cu}$ (Scheme 89a) ${ }^{179} \mathrm{Ni}$ (Scheme 89b) ${ }^{180}$ and $\mathrm{Ru}$ (Scheme $89 \mathrm{c})^{181}$ mediated difluoromethylation of 8-aminoquinoline has been achieved by the $\mathrm{Wu}$, Wang and Zhao groups separately. In 2019, the visible-light-induced difluoroalkylation of 8-aminoquinolines at room temperature was realized by Sun and coworkers (Scheme 90). ${ }^{182} \mathrm{CF}_{2} \mathrm{CO}_{2}$ Et radical

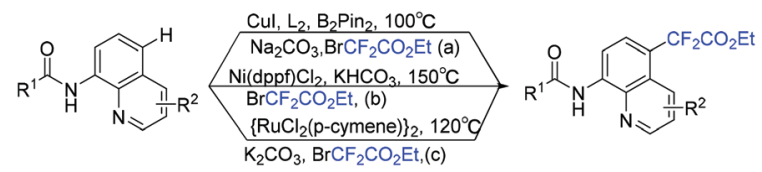

Scheme 89 The $\mathrm{Cu}_{-}, \mathrm{Ni}-$, Ru-catalyzed difluoroalkylation of 8-aminoquinolines.

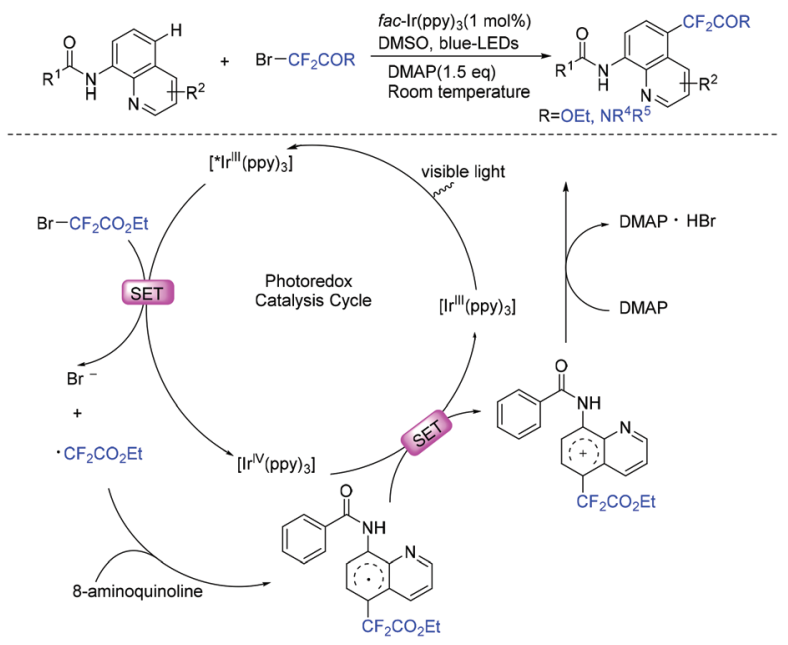

Scheme 90 The visible-light-induced

difluoroalkylation

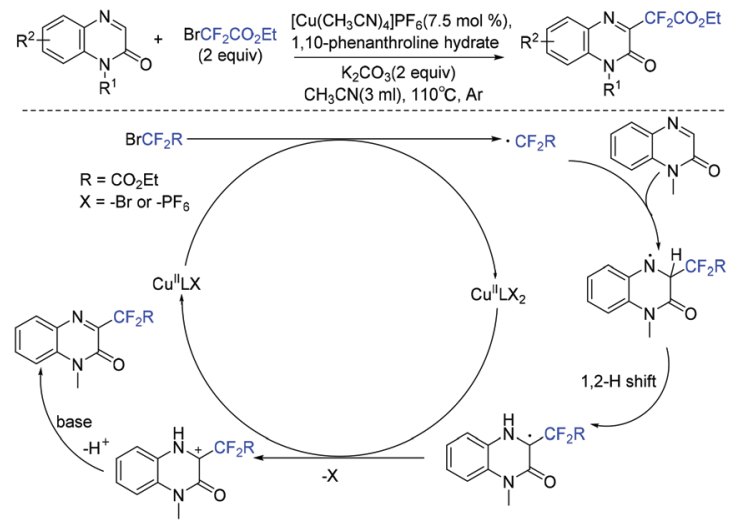

Scheme 91 The copper-catalyzed direct C-3 difluoroacetylation of quinoxalinones.

which was generated from oxidative quenching of excited $\operatorname{Ir}(\mathrm{ppy})_{3}{ }^{*}$ by $\mathrm{BrCF}_{2} \mathrm{CO}_{2}$ Et was proposed in this reaction.

Quinoxalin-2-one derivatives are useful molecules and appear in many bioactive molecules. ${ }^{183-186}$ In 2019 , a coppercatalyzed direct C-3 difluoroacetylation of quinoxalinones with ethyl bromodifluoroacetate was demonstrated by Zhang and coworkers (Scheme 91) ${ }^{187}$ In 2019, the visible-light- and organoamine-cocatalyzed difluoroalkylation of quinoxalinones with functionalized difluoromethyl bromides was also realized by the Sun group (Scheme 92). ${ }^{188}$ Catalytic amount of DIPEA should be added for the success of this transformation. $\mathrm{RCF}_{2}$ radical generated from the reduction of $\mathrm{RCF}_{2} \mathrm{Br}$ was proposed for both of these reactions.

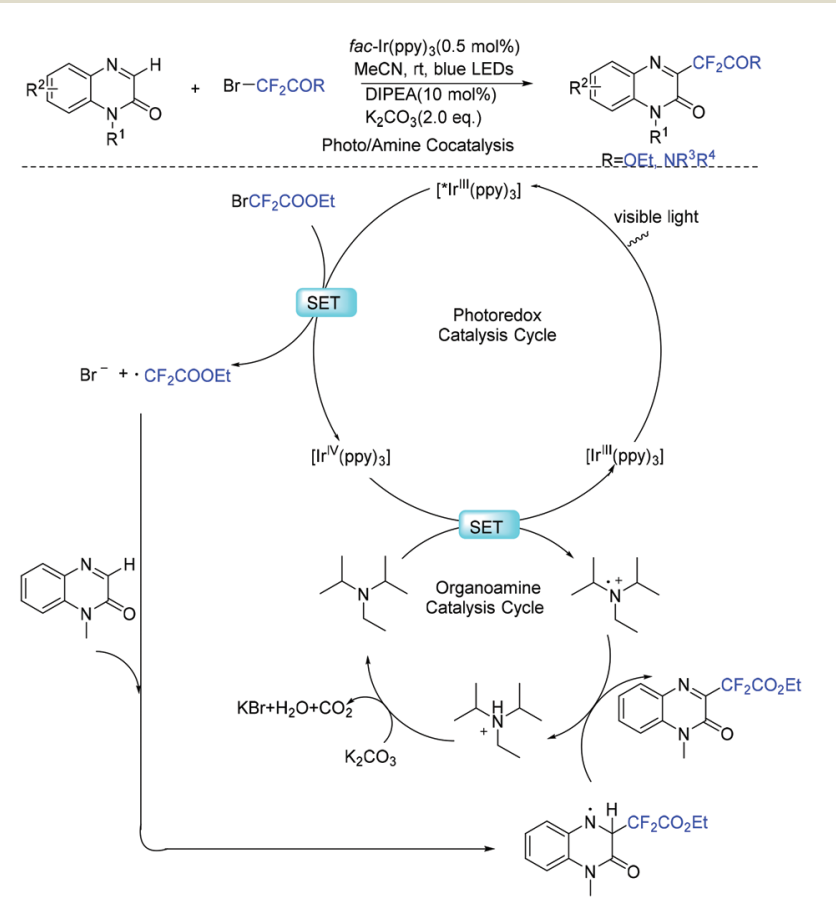

Scheme 92 The visible-light- and organoamine-cocatalyzed difluoroalkylation of quinoxalinones. 
In the same year, the decarboxylative difluoroarylmethylation of quinoxalin-2( $1 H)$-ones with $\alpha, \alpha$-difluoroarylacetic acids was described by the Zhang group (Scheme 93). ${ }^{189}$ The $\mathrm{CF}_{2}$ group of $\alpha, \alpha$-difluoroarylacetic acids was crucial in this transformation. The decarboxylation process between $\alpha, \alpha$-difluorophenylacetic acid and $\left(\mathrm{NH}_{4}\right)_{2} \mathrm{~S}_{2} \mathrm{O}_{8}$ occurred to generate the $\mathrm{Rf}$ radical with the liberation of $\mathrm{CO}_{2}$. The Wan group demonstrated that difluoroarylacetic acids could also react well with other N-heteroarenes (Scheme 94). ${ }^{190}$ Using Ag catalyst, $\mathrm{N}$-heteroarenes such as pyrazines, pyridine, quinoxaline, quinoline, and phenanthridine reacted smoothly in this reaction. The SET between difluoroacetic acid and $\mathrm{Ag}$ (II) which was generated from the oxidation of $\mathrm{Ag}(\mathrm{I})$ by $\left(\mathrm{NH}_{4}\right)_{2} \mathrm{~S}_{2} \mathrm{O}_{8}$ occurred to produce the corresponding nucleophilic aryldifluoromethyl radical.

The first iron-catalyzed cross-coupling of Grignard reagents with fluoroalkyl halides was demonstrated by the Zhang group (Scheme 95). ${ }^{191}$ A diamine, in which a butylene was substi-

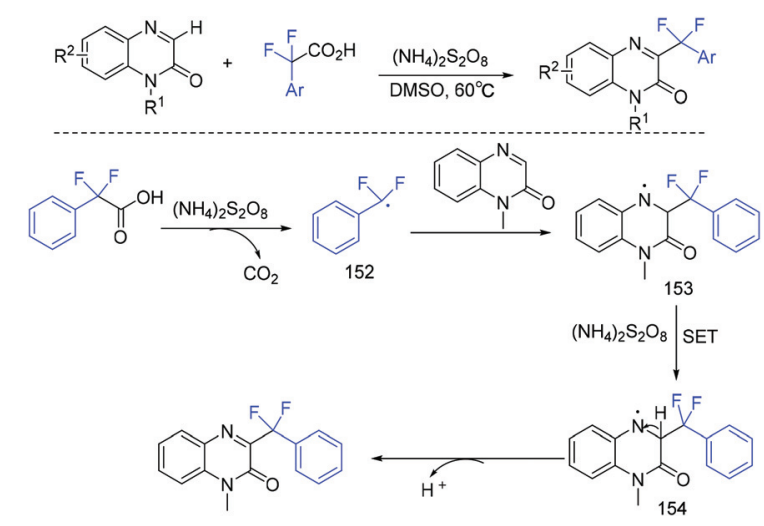

Scheme 93 The decarboxylative difluoroarylmethylation of quinoxalin$2(1 H)$-ones.

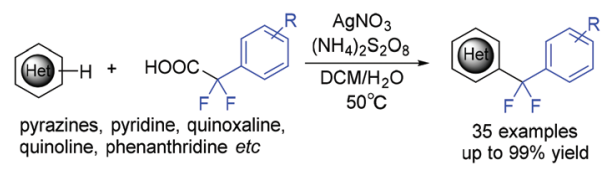

Scheme 94 The decarboxylative difluoroarylmethylation of $\mathrm{N}$-heteroarenes.

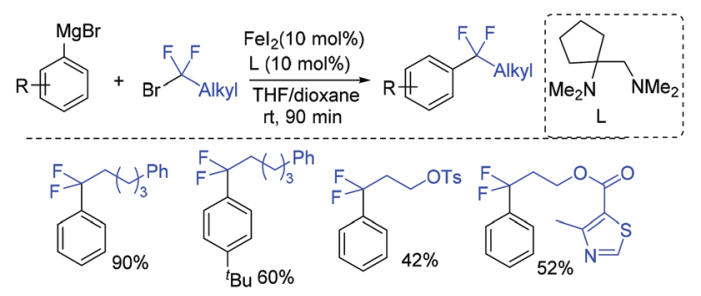

Scheme 95 The cross-coupling of Grignard reagents with fluoroalkyl halides. tuted at one carbon atom of the ethylene backbone in TMEDA, can improve the catalytic efficiency and suppress the side reactions of defluorination, because of the changes of iron complex from five-coordinate to more electron-deficient fourcoordinate.

In 2019, the visible-light-induced difluoroalkylation of arenes with difluoroalkylation reagents $\left(\mathrm{BrCF}_{2} \mathrm{CO}_{2} \mathrm{Et}\right.$ or $\mathrm{BrCF}_{2} \mathrm{CONR}_{1} \mathrm{R}_{2}$ ) was realized by the Liu group (Scheme 96). ${ }^{192}$ The in situ formation of cuprous photocatalyst from cuprous iodide, an imine ligand and a triaryl phosphine ligand was a key factor for this reaction. Difluoromethyl radicals which were generated via SET from the excited photocatalyst to difluoroalkylation reagents was suggested in the difluoroalkylation. In the same year, Hisaeda and coworkers found that these reactions can also be realized in the presence of cobalt catalyst (Scheme 97). ${ }^{193}$ Driven by controlled-potential electrolysis and visible-light irradiation in dimethyl sulfoxide, a wide range of electron-rich arenes and $\mathrm{N}-$, O-, and S-containing heteroarenes

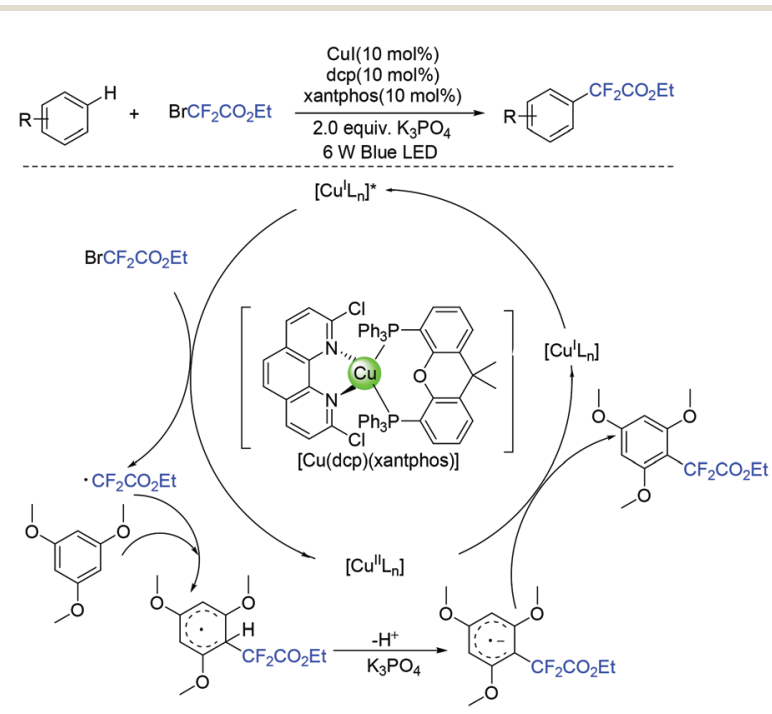

Scheme 96 Visible-light-induced difluoroalkylation of arenes.

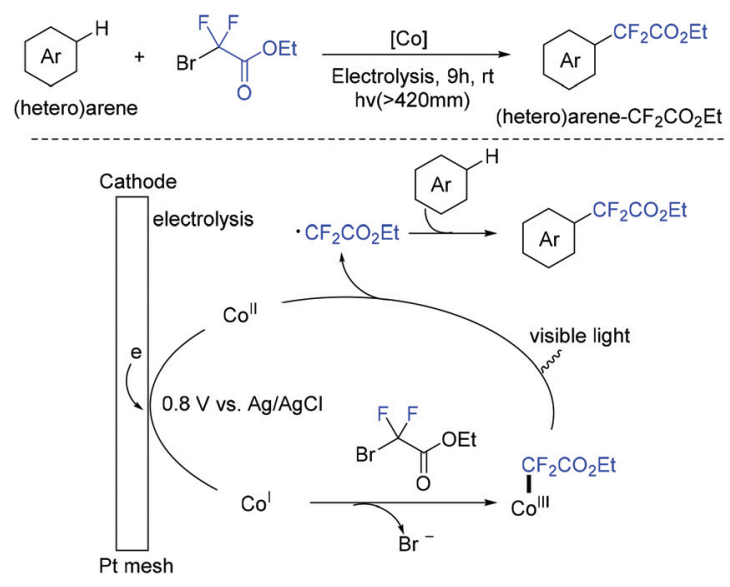

Scheme 97 The Co-catalyzed difluoroalkylation of arenes. 

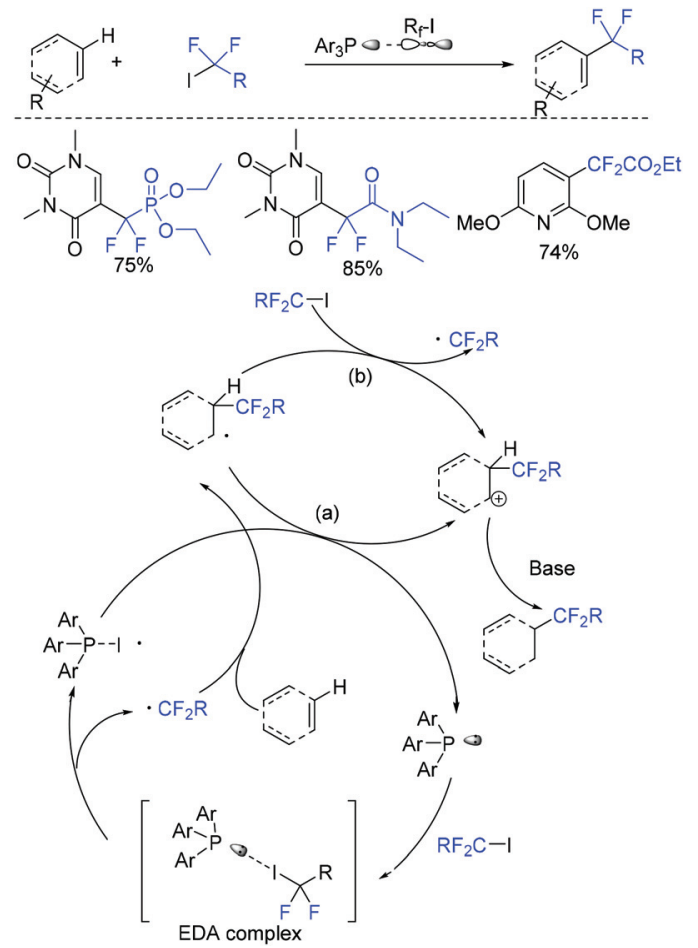

Scheme 98 The triaryl phosphine-catalyzed difluoroalkylation of arenes.

reacted smoothly. Mechanistic studies revealed that a radical pathway mediated by homolytic cleavage of the cobalt(III) was involved in the mechanism. In the presence of triaryl phosphine as the catalyst, the visible-light-promoted difluoroalkylation reaction of arenes or heterocycles with difluoroalkyl iodide was presented by the Zhang group (Scheme 98). ${ }^{180}$ Mechanistic investigation implied that a radical-chain process that was initiated by an electron donor-acceptor complex formed from difluoroalkyl iodide and phosphine was involved in this reaction.

An efficient method for the synthesis of 3,3-difluoro-2-oxindoles via copper/ $\mathrm{B}_{2} \mathrm{pin}_{2}$-catalyzed $\mathrm{C}-\mathrm{H}$ difluoroacetylationcycloamidation of anilines with ethyl bromodifluoroacetate was realized by the Song group (Scheme 99). ${ }^{194}$ In this strategy, amino groups act as directing groups, providing ortho-difluoroacetylated products regioselectively. In the presence of a base, $\mathrm{LCu}(\mathrm{I})$-Bpin species 155 was generated between $\operatorname{LCuX}(\mathrm{I})$ and $\mathrm{B}_{2} \mathrm{pin}_{2}$. Then $\mathrm{BrCF}_{2} \mathrm{CO}_{2} \mathrm{R}$ was reduced by species 155 to give a $\mathrm{CF}_{2} \mathrm{CO}_{2} \mathrm{R}$ radical. Subsequently $\mathrm{CF}_{2} \mathrm{CO}_{2} \mathrm{R}$ radical reacted with aniline to deliver intermediate $\mathbf{1 5 7}$ directed by amino groups. Finally, the 3,3-difluoro-2-oxindole derivatives were obtained after a SET pathway and intramolecular cycloamidation between ester and amino groups.

In the same year, the Zhang group reported a visible-lightinduced photoredox difluoromethylation-amidation of anilines with bromodifluoroacetate (Scheme 100). ${ }^{195} \mathrm{~A}$ broad range of difluorooxindoles was obtained via this process. However, aniline was not suitable for this system owing to the

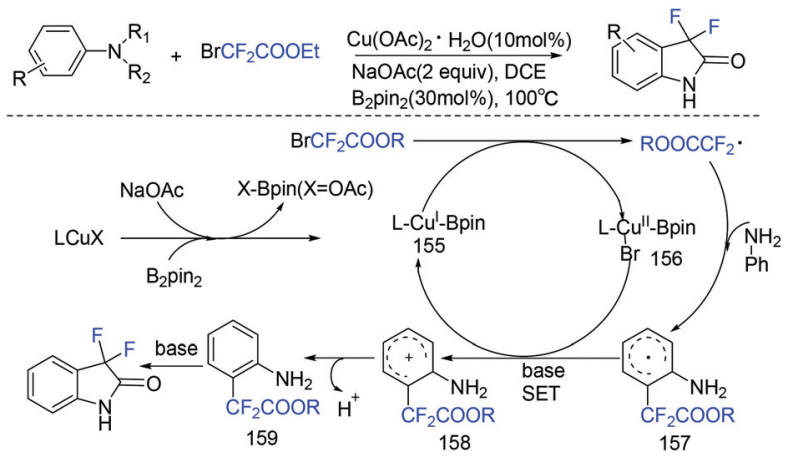

Scheme 99 The copper/B ${ }_{2}$ pin $_{2}$-catalyzed $\mathrm{C}-\mathrm{H}$ difluoroacetylationcycloamidation of anilines.

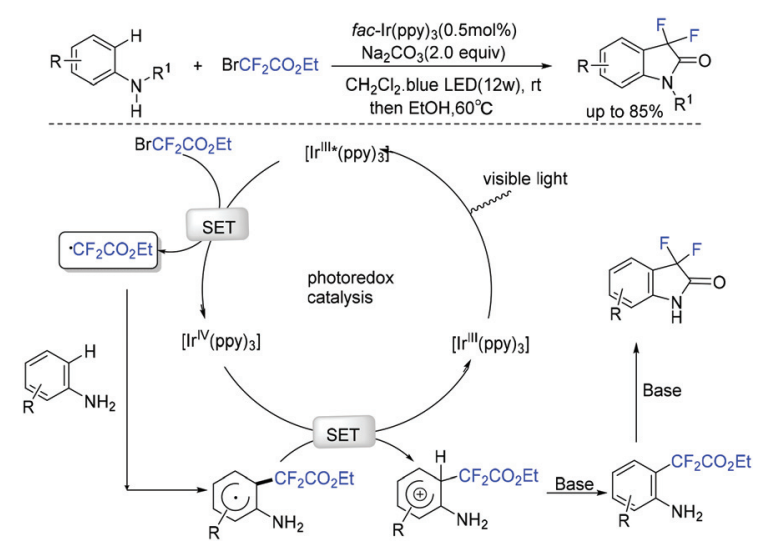

Scheme 100 The visible-light-induced photoredox difluoromethylation-amidation of anilines.

formation of a large amount of para-difluoroacetylated aniline. In the catalytic cycle, the reaction of bromodifluoroacetate and excited state of the photocatalyst ${ }^{*}\left[\operatorname{Ir}(\mathrm{ppy})_{3}\right]$ was also proposed for this transformation to afford $\mathrm{RCF}_{2}$ radical. The Sun group found that 2 -(1H-pyrrol-1-yl)anilines and 2-(1H-indol-1-yl)anilines were also good substrates to react with bromodifluoroacetate, providing various fluorinated pyrrolo[1,2- $d]$ benzodiazepine derivatives. ${ }^{196}$

Because of the steric disadvantage as well as entropy, the use of aryl-substituted anilines for this difluoromethylation transformation was challenging. The Li group realized the copper-catalyzed C-H [3 + 2] annulation of $\mathrm{N}$-aryl- or alkyl-substituted anilines with bromodifluoroacetate (Scheme 101). ${ }^{197}$ The corresponding 3,3'-disubstituted oxindole products with moderate to good yields were assembled. In 2019, the Pd-cata-

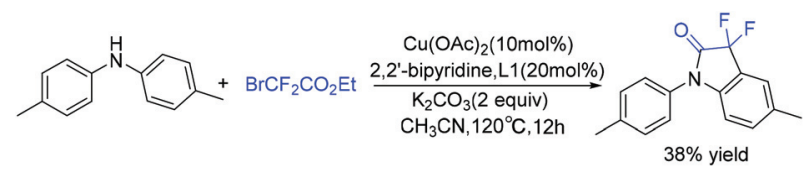

Scheme 101 The copper-catalyzed annulation of $N$-substituted anilines. 


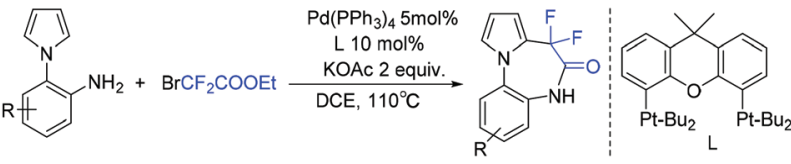

Scheme 102 The copper-catalyzed annulation of $\mathrm{N}$-substituted anilines.

lyzed two-component $\mathrm{C}-\mathrm{H}$ difluoroalkylation cyclization cascade reaction of 1-(2-aminophenyl)pyrrole derivatives was realized by the Liu group (Scheme 102). ${ }^{198}$ A variety of difluoroalkylated pyrrolobenzodiazepine derivatives were obtained in good yields. Two possible pathways which involved radical intermediates were proposed for this transformation.

\section{Difluoroalkylation with alkyl $\mathrm{C}-\mathrm{H}$ bond}

Because of the high bond dissociation energy and lack of "active" HOMO or LUMO orbitals to interact with transition metal catalytic centers, it is more difficult to functionalize a C(sp3)-H bond. ${ }^{199-204}$ However, rapid progress has been achieved in recent years. ${ }^{133,205-210}$ Ketones are some of the most commonly used substrates for fluoroalkylation reactions. $^{211,212}$ However, defluorination reactions often occur to form fluoroalkene compounds. In order to prevent defluorination reactions, tertiary ketones are usually necessary. In 2018, the group of $\mathrm{Lu}$ and Xiao described enantioselective radical difluoroalkylation reactions of $\beta$-ketoesters using an asymmetric photoredox and nickel catalysis cascade (Scheme 103). ${ }^{213}$ But the corresponding product was isolated with moderate yield and enantioselectivity (55\% yield, 83:17 er) when $\mathrm{BrCF}_{2} \mathrm{CO}_{2} \mathrm{R}$ was used. In addition, perfluoroalkylation of $\beta$-ketoesters was also realized in this system. The reductive quenching of $\operatorname{Ir}(\mathrm{III})^{*}$ with the Ni complex was proposed in the catalytic cycle. $\mathrm{CF}_{2} \mathrm{CO}_{2} \mathrm{Et}$ radical was generated from the reduction of $\mathrm{XCF}_{2} \mathrm{CO}_{2}$ Et by $\operatorname{Ir}(\mathrm{II})$.

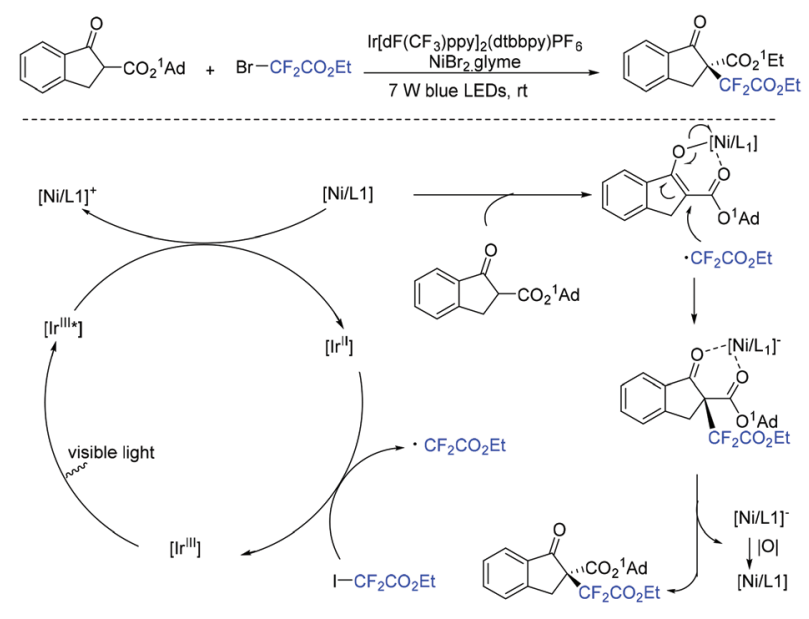

Scheme 103 The difluoroalkylation reactions of $\beta$-ketoesters.
In 2018, the cobalt-catalyzed difluoroalkylation of tertiary aryl ketones for facile synthesis of quaternary alkyl difluorides was disclosed by the Wang group (Scheme 104). ${ }^{214}$ Preliminary mechanistic investigations indicated that a difluoroalkyl radical was involved in a $\mathrm{Co}(\mathrm{I}) / \mathrm{Co}(\mathrm{III})$ catalytic cycle. $\mathrm{Zn}$ metal could reduce the $\mathrm{Co}(\mathrm{II})$ to the catalytically active $\mathrm{Co}(\mathrm{I})$ species. Nickel was also found to be efficient for this type of reaction by the same group the following year (Scheme 105). ${ }^{215}$ But only tertiary aryl ketones were suitable for this difluoroalkylation reaction. A $\mathrm{Ni}(\mathrm{I}) / \mathrm{Ni}(\mathrm{III})$ catalytic cycle was proposed for this reaction. If a secondary ketone was employed, a tetrafluoroalkylated monofluoroalkene product was formed through defluorination.

In 2018, the methoxycarbonyldifluoromethylation of trimethylsilyl enol ethers and allyltrimethylsilanes with $\mathrm{FSO}_{2} \mathrm{CF}_{2} \mathrm{CO}_{2} \mathrm{Me}$ induced by visible light was developed by the
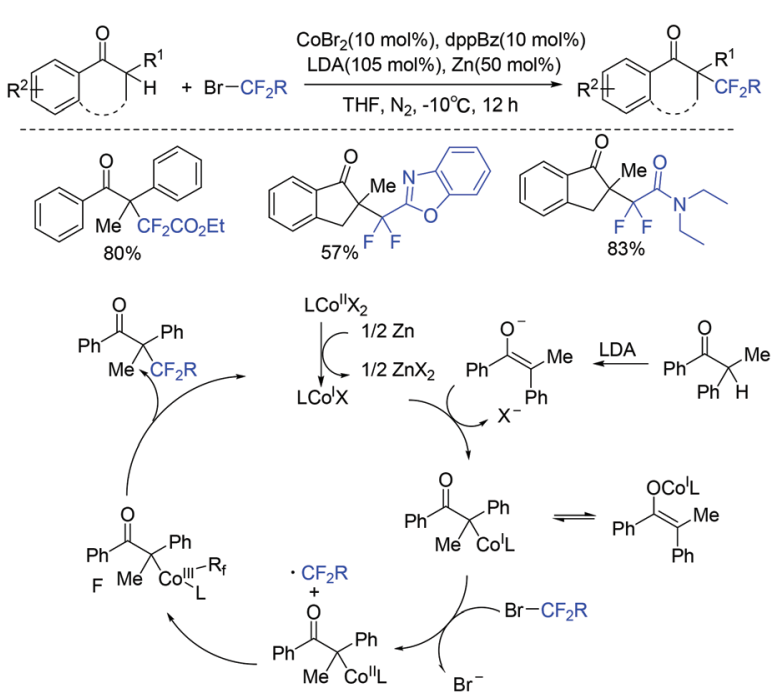

Scheme 104 The cobalt-catalyzed difluoroalkylation of tertiary aryl ketones.

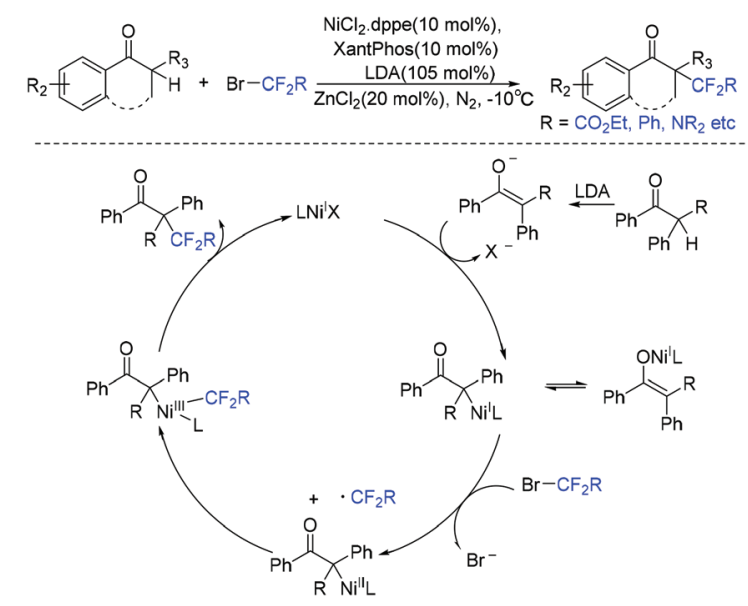

Scheme 105 The nickel-catalyzed difluoroalkylation of tertiary aryl ketones. 

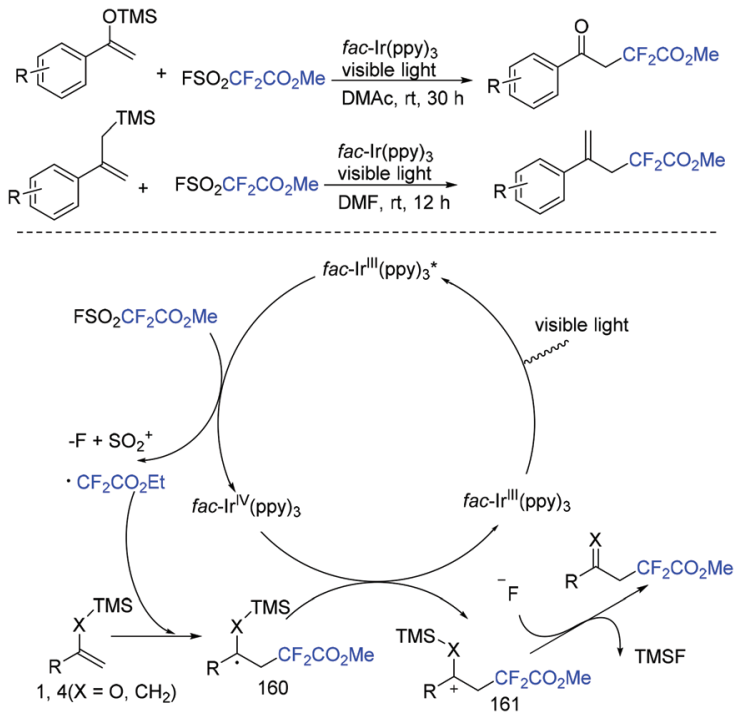

Scheme 106 The methoxycarbonyldifluoromethylation of trimethylsilyl enol ethers.

Qing group (Scheme 106). ${ }^{216}$ Aromatic ketones and aliphatic ketones were all compatible with this transformation. The oxidative quenching of $f a c-\mathrm{Ir}^{\mathrm{III}}(\mathrm{ppy})_{3}{ }^{*}$ with $\mathrm{FSO}_{2} \mathrm{CF}_{2} \mathrm{CO}_{2} \mathrm{Me}$ took place to give $\mathrm{CF}_{2} \mathrm{CO}_{2} \mathrm{Me}$ radical and $f a c-\mathrm{Ir}^{\mathrm{IV}}(\mathrm{ppy})_{3}$ was proposed for this reaction. Additionally, fluoride anion could assist the desilylation of $\mathbf{1 6 1}$ to provide the target products.

\section{Difluoroalkylation with isocyano}

In 2013, the first visible-light-promoted isocyanide insertion was described by the Yu group (Scheme 107). ${ }^{217}$ Various 6-alkylated phenanthridine derivatives were obtained with good to excellent yields. Fluoroalkyl radical which was generated from the reduction of ethyl bromodifluoroacetate by $\operatorname{Ir}(\mathrm{ppy})_{3}{ }^{*}$ added with isocyanide to provide radical $\mathbf{1 6 2}$. Subsequently, radical 162 underwent intramolecular hemolytic

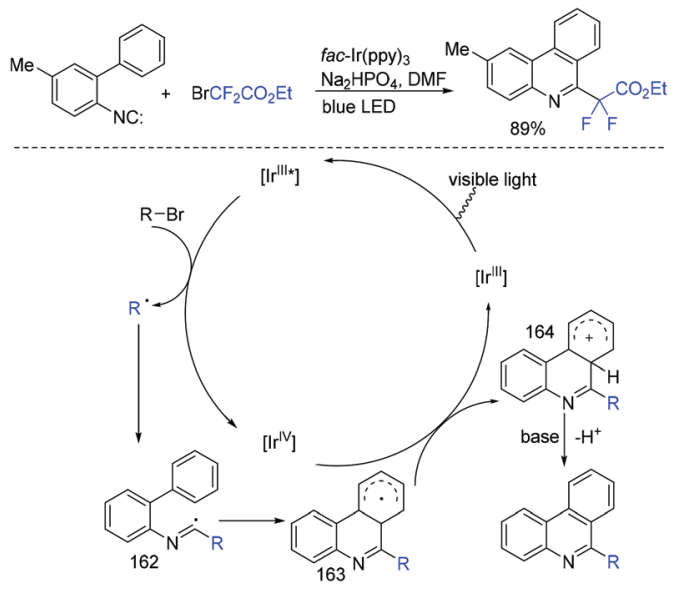

Scheme 107 The visible-light-promoted isocyanide insertion. aromatic substitution to give radical intermediate 163. Then radical intermediate $\mathbf{1 6 3}$ was followed by oxidation and deprotonation reaction to release the desired product.

The Song group realized the sodium sulfite-involved photocatalytic radical cascade cyclization of 2-isocyanoarylthioethers (Scheme 108). ${ }^{218}$ 2- $\mathrm{CF}_{2}$-containing benzothiazoles can be efficiently prepared using this methodology. Different from previous reports, sodium sulfite should be added in the photocatalytic cycle to reduce $\operatorname{Ir}^{\mathrm{IV}}$ into $\mathrm{Ir}^{\mathrm{III}}$.

$\mathrm{A} \mathrm{Cu} / \mathrm{B}_{2} \mathrm{pin}_{2}$ system-promoted cyclization reaction of vinyl isocyanides with bromodifluoroacetic derivatives for the synthesis of 1-difluoroalkylated isoquinolines was reported by the Shi group (Scheme 109). ${ }^{219}$ Preliminary mechanistic studies revealed that a tandem radical cyclization process was involved in this transformation. In addition, this transformation can also be induced by visible light.

\section{Difluoroalkylation with amine}

In 2018, the Song group found that $\mathrm{BrCF}_{2} \mathrm{CO}_{2} \mathrm{Et}$ played a dual role as a $\mathrm{C} 1$ synthon and a difluoroalkylating reagent in the reaction of $\mathrm{BrCF}_{2} \mathrm{CO}_{2} \mathrm{Et}$ with primary amines (Scheme 110). ${ }^{220}$ Difluorocarbene which was generated from the reaction of $\mathrm{BrCF}_{2} \mathrm{CO}_{2}$ Et with $\mathrm{Na}_{2} \mathrm{CO}_{3}$ via decarboxylation and debromination processes was involved in the catalytic cycle. Based on

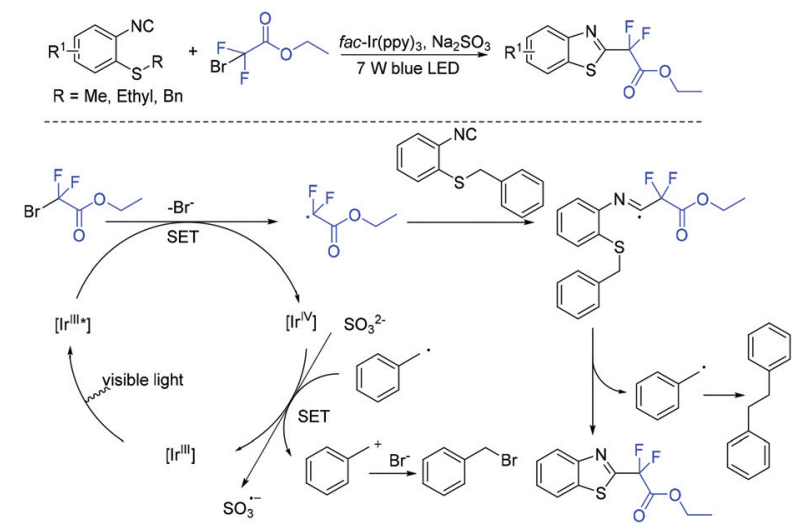

Scheme 108 The photocatalytic radical cascade cyclization of 2-isocyanoarylthioethers.

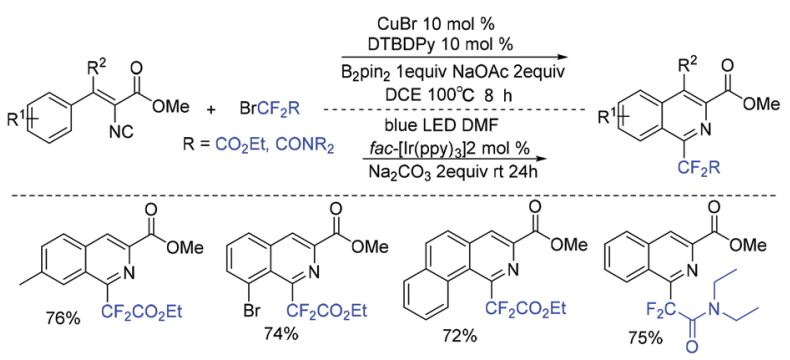

Scheme 109 The $\mathrm{Cu} / \mathrm{B}_{2} \mathrm{pin}_{2}$-promoted cascade cyclization of vinyl isocyanides. 


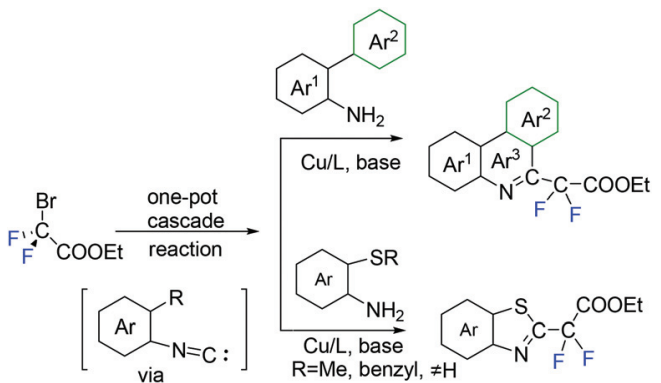

Scheme 110 The reaction of $\mathrm{BrCF}_{2} \mathrm{COOEt}$ with primary amines.

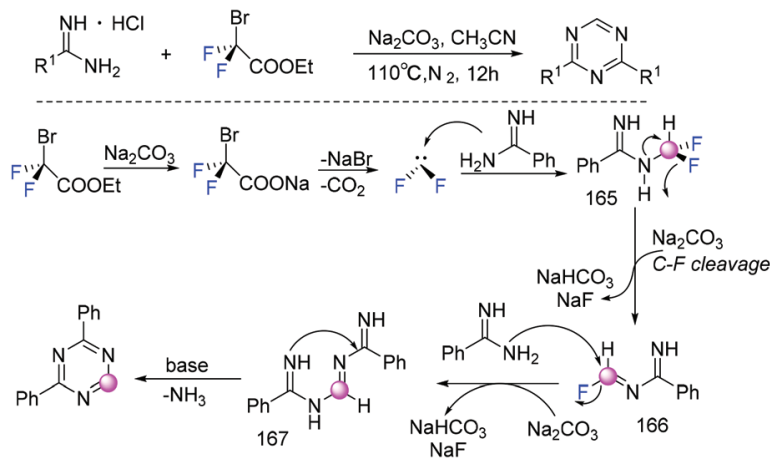

Scheme 111 The synthesis of 2,4-disubstituted 1,3,5-triazines.

experimental observations and DFT calculations, they found that base played an active role in the formation of the key intermediate isocyanides generated from primary amines and difluorocarbene. The same group found that ethyl bromodifluoroacetate could also serve as a C1 synthon to access 2,4-disubstituted 1,3,5-triazines (Scheme 111). ${ }^{221}$ Both symmetric and unsymmetric 2,4-disubstituted 1,3,5-triazines could be obtained with only small amounts of toxic inorganic wastes generated. Based on experimental results, it was speculated that difluorocarbene $\left(: \mathrm{CF}_{2}\right)$ was actually generated from $\mathrm{BrCF}_{2} \mathrm{CO}_{2} \mathrm{Et}$ with the assistance of base. Then difluorocarbene $\left(: \mathrm{CF}_{2}\right)$ reacted with amidine to afford intermediate 165 , which was defluorinated to imine intermediate 166 under basic conditions. Subsequently, intermediate $\mathbf{1 6 7}$ was generated from the reaction of intermediate $\mathbf{1 6 6}$ with another amidine. Finally the corresponding products were formed through intramolecular nucleophilic addition of $\mathbf{1 6 7}$ and elimination of one molecule of ammonia.

\section{Difluoroalkylation with $\mathrm{C}=\mathrm{N}$}

In 2016, the CuI (Scheme 112a) $)^{222}$ and $\operatorname{Pd}_{2}(\mathrm{dba})_{3}$ (Scheme 112b) ${ }^{223}$ catalyzed difluoroalkylations of hydrazones were realized by the Monteiro group. The Ir (Scheme 112c) ${ }^{224}$ and $\mathrm{Au}$ (Scheme 112d) ${ }^{225}$ catalyzed difluoroalkylation of hydrazones induced by visible light was also presented by the Zhu and Hashmi groups separately. The $\mathrm{Cu}(\mathrm{II}) / \mathrm{B}_{2} \mathrm{pin}_{2}$-catalyzed

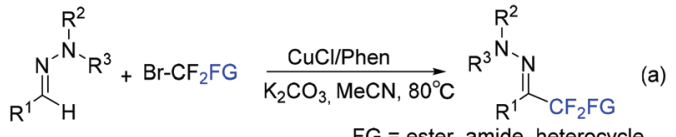

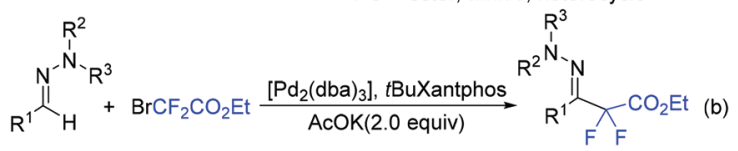

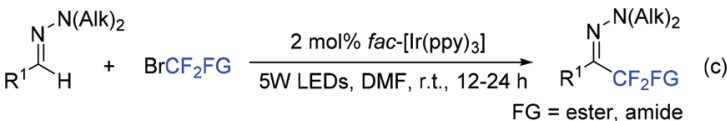

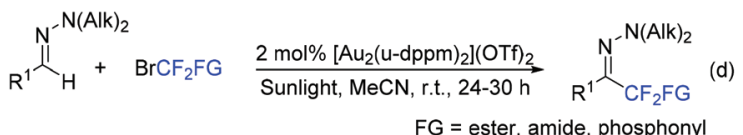

Scheme 112 The difluoroalkylation of hydrazones catalyzed by various metals.

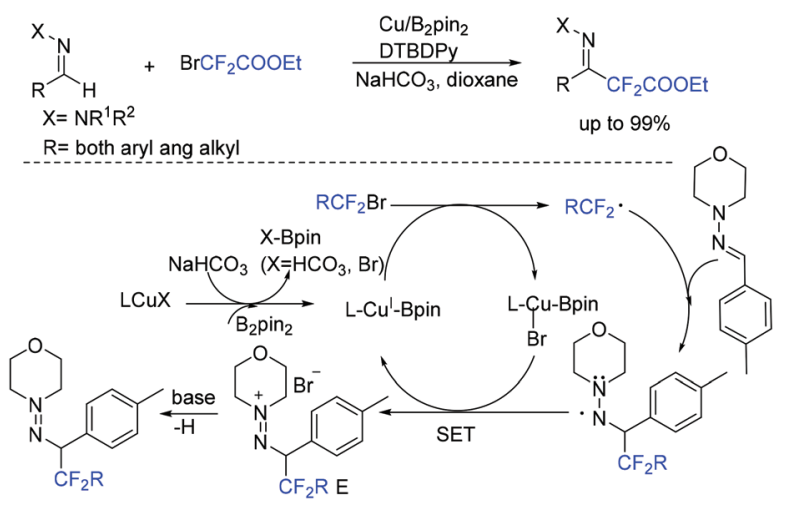

Scheme 113 The $\mathrm{Cu} / \mathrm{B}_{2}$ pin $_{2}$-catalyzed difluoroalkylation of hydrazones.

difluoroalkylation of hydrazones with difluoroalkyl bromides using diboron as reductant was reported the Song group (Scheme 113). ${ }^{226}$ Both aromatic and aliphatic hydrazones were suitable for this reaction. A difluoroalkyl radical via a SET pathway was suggested in the reaction mechanism. Additionally, the catalytic diboron reagent was a key factor in this transformation.

The direct three-component difluoroalkylation reaction between aldehydes, hydrazines, and halodifluoromethylated reagents catalyzed by visible-light organic photoredox was realized by Wang and coworkers (Scheme 114). ${ }^{227}$ A reductive quenching of photoexcited $\mathrm{PC}^{*}$ species with the hydrazine in situ generated from aldehyde and hydrazine to form the strong reductant $\mathrm{PC}^{-}$and radical intermediate 168 was proposed for this reaction.

In 2019, a visible-light-induced difluoroalkylation of $\mathrm{N}, \mathrm{N}$ cyclicazomethine imine with $\mathrm{BrCF}_{2} \mathrm{R}$ was successfully realized by the Yang group (Scheme 115). ${ }^{228}$ Mechanistic investigations showed that a reductive radical-radical coupling pathway and oxidation of ascorbic acid with Ir(Iv) to form ascorbyl radical were involved in the mechanism. 

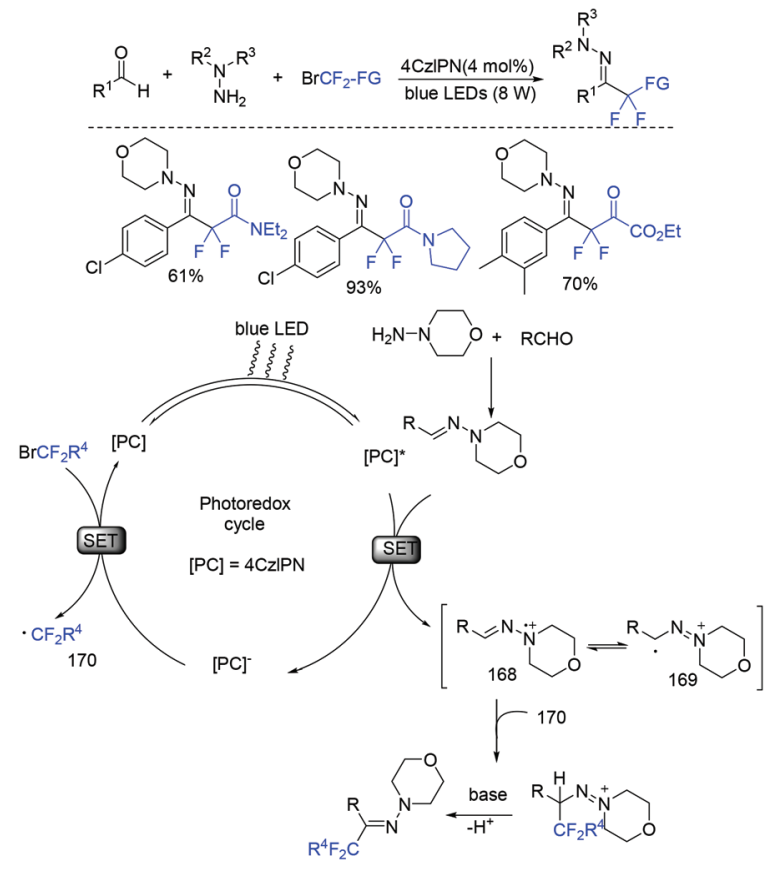

Scheme 114 The three-component difluoroalkylation reaction between aldehydes, hydrazines and halodifluoromethylated reagents.

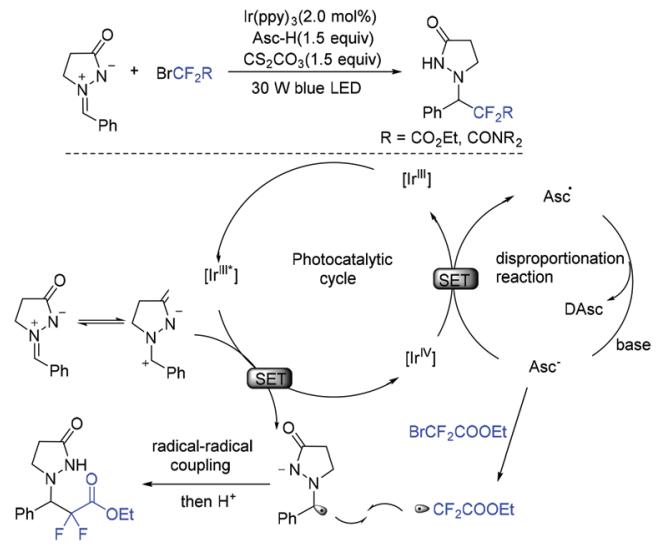

Scheme 115 The difluoroalkylation of $N, N$-cyclicazomethine imine.

The first base-catalyzed enantioselective phosphonyldifluoromethylation was described by the Vilotijevic group (Scheme 116). ${ }^{229}$ By the use of diethyl(difluoro(trimethylsilyl) methyl)phosphonate reagent as a latent pronucleophile, the enantiomers of the product can be readily accessed. A kinetic resolution was involved in the reaction process. But the yield of the reaction was not very high.

$$
\underset{+\mathrm{TMSCF}_{2} \mathrm{P}(\mathrm{O})(\mathrm{OEt})_{2}}{\mathrm{CO}_{2} \mathrm{R}^{1}} \stackrel{(\mathrm{DHQD})_{2} \mathrm{PHAL}(10 \% \mathrm{mmol})}{\text { dioxane/THF }}
$$

Scheme 116 Enantioselective substitution of allylic fluorides.

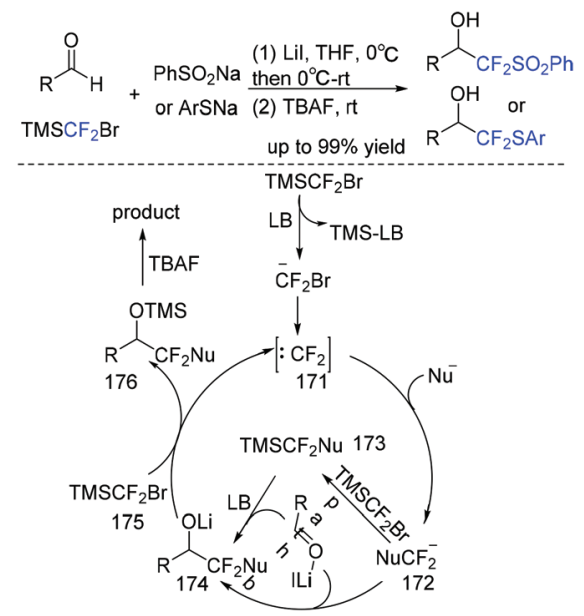

Scheme 117 The (phenylsulfonyl)difluoromethylation of aldehydes.

\section{Difluoroalkylation with $\mathrm{C}=\mathrm{O}$}

In the past decade, tremendous efforts have been made in (phenylsulfonyl/arylthio)difluoromethylation of aldehydes. $^{230-234}$ Among these reactions, the nucleophilic addition of $\mathrm{PhSO}_{2} \mathrm{CF}_{2}{ }^{-}$or $\mathrm{PhSCF}_{2}^{-}$anion to carbonyl compounds (such as aldehydes) was the most popular method. In 2019, the $\mathrm{Hu}$ group found that $\mathrm{TMSCF}_{2} \mathrm{Br}$ was good partner for nucleophilic (phenylsulfonyl/arylthio)difluoromethylation of aldehydes and $\mathrm{PhSO}_{2} \mathrm{Na}(\mathrm{ArSNa})$ (Scheme 117). ${ }^{235}$ The preparation of $\mathrm{PhSO}_{2} \mathrm{CF}_{2} \mathrm{X}$ or $\mathrm{PhSCF}_{2} \mathrm{X}(\mathrm{X}=\mathrm{H}, \mathrm{Br}$, TMS) reagents was avoided in this three-component reaction. The in situ generation of difluorocarbene via the reaction of $\mathrm{TMSCF}_{2} \mathrm{Br}$ with a Lewis base activator $\left(\mathrm{LB}=\mathrm{PhSO}_{2}-\right.$, $\mathrm{PhS}-$, or 174) was proposed in the reaction mechanism. Then the reaction of difluorocarbene with $\mathrm{PhSO}_{2} \mathrm{Na}(\mathrm{ArSNa})$ occurred to form the corresponding $\mathrm{PhSO}_{2} \mathrm{CF}_{2}\left(\mathrm{PhSCF}_{2}\right)$ anions. Finally, the desired difluoromethylated products were obtained via nucleophilic addition of $\mathrm{PhSO}_{2} \mathrm{CF}_{2}\left(\mathrm{PhSCF}_{2}\right)$ anions to aldehydes.

\section{Conclusions}

The difluoroalklyation reaction has made much progress in the last few years. In this review, we summarized the advances of difluoroalklyation reactions in the last three years. The most commonly used difluoroalkylation reagent was $\mathrm{RCF}_{2} \mathrm{X}$. Generally speaking, $\mathrm{RCF}_{2}$ radical which was usually generated from the reduction of $\mathrm{RCF}_{2} \mathrm{X}$ was the active intermediate in most cases. When alkenes or alkynes were used as substrates, reactions were usually initiated by radical addition to the carbon-carbon unsaturated bonds; then the final products can be isolated in different ways. Visible-light-induced and metalcatalyzed difluoroaklyations were all realized. Some electronrich bases could also be used to induce the formation of $\mathrm{RCF}_{2}$ radical for these reactions via EDA complex. At present, the most efficient photocatalyst was metal [Ir]. In the absence of visible light, copper was proved to be one of the most effective 
catalysts in metal catalyzed difluoroalkylation. In consideration of green chemistry and cost efficiency, a cheap photocatalyst or even catalyst-free difluoroalkylation reaction is more desirable. Asymmetric difluoroalkylation is also worth further study. Additionally, multicomponent reaction for the introduction of $\mathrm{RCF}_{2}$ group also needs to be further explored. We anticipate that this review will open new avenues for the development of difluoroalkylation reactions.

\section{Conflicts of interest}

There are no conflicts to declare.

\section{Acknowledgements}

This work was supported by the National Natural Science Foundation of China (no. 21772107) and Shandong Province Key Research and Development Plan (no. 2019GSF108017). We also thank Cai-Zhen Ding, Yan-Li Wang and Hong-Di Yang for their useful help.

\section{Notes and references}

1 A. Vulpetti and C. Dalvit, Fluorine local environment: from screening to drug design, Drug Discovery Today, 2012, 17, 890-897.

2 A. D. Dilman and V. V. Levin, Difluorocarbene as a Building Block for Consecutive Bond-Forming Reactions, Acc. Chem. Res., 2018, 51, 1272-1280.

3 T. Lasing, A. Phumee, P. Siriyasatien, K. Chitchak, P. Vanalabhpatana, K. K. Mak, C. Hee Ng, T. Vilaivan and T. Khotavivattana, Synthesis and antileishmanial activity of fluorinated rhodacyanine analogues: The 'fluorinewalk' analysis, Bioorg. Med. Chem., 2020, 28, 115187.

4 Z. Feng, Y. L. Xiao and X. G. Zhang, Transition-Metal (Cu, Pd, Ni)-Catalyzed Difluoroalkylation via Cross-Coupling with Difluoroalkyl Halides, Acc. Chem. Res., 2018, 51, 2264-2278.

5 W. Wang, Q. Yu, Q. Zhang, J. Li, F. Hui, J. Yang and J. Lü, Recent Progress on Difluoromethylation Methods, Chin. J. Org. Chem., 2018, 38, 1569-1585.

6 X. F. Tao, R. Sheng, K. Bao, Y. X. Wang and Y. X. Jin, Progress of Difluoromethyl Heteroaryl Sulfones as Difluoroalkylation Reagents, Chin. J. Org. Chem., 2019, 39, 2726-2734.

7 A. Lemos, C. Lemaire and A. Luxen, Progress in Difluoroalkylation of Organic Substrates by Visible Light Photoredox Catalysis, Adv. Synth. Catal., 2019, 361, 15001537.

8 X. S. Hu, J. S. Yu and J. Zhou, Catalytic selective monoand difluoroalkylation using fluorinated silyl enol ethers, Chem. Commun., 2019, 55, 13638-13648.

9 J. Mizukado, Y. Matsukawa, H.-D. Quan, M. Tamura and A. Sekiya, Reactions of aliphatic fluoro-alcohols with
$\mathrm{CHClF}_{2}$ at atmospheric pressure, J. Fluorine Chem., 2006, 127, 400-404.

10 (a) R. M. Flynn and D. J. Burton, Synthetic and mechanistic aspects of halo-F-methylphosphonates, J. Fluorine Chem., 2011, 132, 815-828; (b) Z. Feng, F. Chen and $\mathrm{X}$. Zhang, Copper catalyzed cross-coupling of iodobenzoates with bromozinc-difluorophosphonate, Org. Lett., 2012, 14, 1938-1941.

11 O. V. Fedorov, M. I. Struchkova and A. D. Dilman, Silicon Reagent with Functionalized Tetrafluoroethylene Fragments: Preparation and Coupling with Aldehydes, J. Org. Chem., 2016, 81, 9455-9460.

12 X. Song, S. Tian, Z. Zhao, D. Zhu and M. Wang, Controlled Ring-Opening of Siloxydifluorocyclopropanes for Carbocyclization: Synthesis of Difluorocyclopentenones, Org. Lett., 2016, 18, 3414-3417.

13 J. Mizukado, Y. Matsukawa, H.-D. Quan, M. Tamura and A. Sekiya, Insertion reactions of difluorocarbene generated by pyrolysis of hexafluoropropene oxide to $\mathrm{OH}$ bond, J. Fluorine Chem., 2005, 126, 365-369.

14 K. Levchenko, O. P. Datsenko, O. Serhiichuk, A. Tolmachev, V. O. Iaroshenko and P. K. Mykhailiuk, Copper-Catalyzed O-Difluoromethylation of Functionalized Aliphatic Alcohols: Access to Complex Organic Molecules with an $\mathrm{OCF}_{2} \mathrm{H}$ Group, J. Org. Chem., 2016, 81, 5803-5813.

15 S. Krishnamoorthy, J. Kothandaraman, J. Saldana and G. K. S. Prakash, Direct Difluoromethylenation of Carbonyl Compounds by Using $\mathrm{TMSCF}_{3}$ : The Right Conditions, Eur. J. Org. Chem., 2016, 4965-4969.

16 Y. Zhao, W. Huang, J. Zheng and J. Hu, Efficient and Direct Nucleophilic Difluoromethylation of Carbonyl Compounds and Imines with $\mathrm{Me}_{3} \mathrm{SiCF}_{2} \mathrm{H}$ at Ambient or Low Temperature, Org. Lett., 2011, 13, 5342-5345.

17 Y. Arai, R. Tomita, G. Ando, T. Koike and M. Akita, Oxydifluoromethylation of Alkenes by Photoredox Catalysis: Simple Synthesis of $\mathrm{CF}_{2} \mathrm{H}$-Containing Alcohols, Chem. - Eur. J., 2016, 22, 1262-1265.

18 (a) K. Aikawa, H. Serizawa, K. Ishii and K. Mikami, Palladium-Catalyzed Negishi Cross-Coupling Reaction of Aryl Halides with (Difluoromethyl)zinc Reagent, Org. Lett., 2016, 18, 3690-3693; (b) X. T. Huang, Z. Y. Long and Q. Y. Chen, Fluoroalkylation of aromatic compounds with per(poly)fluoroalkyl chlorides initiated by sodium dithionite in DMSO, J. Fluorine Chem., 2001, 111, 107-113.

19 (a) W. Liu, W. Yan, M. Paeth, S. Zacate and X. Zeng, LateStage Difluoromethylation of Aliphatic Carboxylic Acids with Copper Catalysis, Synlett, 2020, 745-749; (b) C. Xu, W.-H. Guo, X. He, Y.-L. Guo, X.-Y. Zhang and X. Zhang, Difluoromethylation of (hetero)aryl chlorides with chlorodifluoromethane catalyzed by nickel, Nat. Commun., 2018, 9, 1170 .

20 X. Xiao, Z.-T. Zheng, T. Li, J.-L. Zheng, T. Tao, L.-M. Chen, J.-Y. Gu, X. Yao, J.-H. Lin and J.-C. Xiao, Recent Advances in Difluoromethylthiolation, Synthesis, 2020, 197-207. 
21 T. Koike and M. Akita, Recent progress in photochemical radical di- and mono-fluoromethylation, Org. Biomol. Chem., 2019, 17, 5413-5419.

22 (a) N. Levi, D. Amir, E. Gershonov and Y. Zafrani, Recent Progress on the Synthesis of CF2H-Containing Derivatives, Synthesis, 2019, 4549-4567; (b) Z. Feng, Q.-Q. Min, X.-P. Fu, L. An and X. Zhang, Chlorodifluoromethane-triggered formation of difluoromethylated arenes catalysed by palladium, Nat. Chem., 2017, 9, 918-923.

23 T. Y. Shang, L. H. Lu, Z. Cao, Y. Liu, W. M. He and B. Yu, Recent advances of 1,2,3,5-tetrakis(carbazol-9-yl)-4,6dicyanobenzene (4CzIPN) in photocatalytic transformations, Chem. Commun., 2019, 55, 5408-5419.

24 Y. Chen, L.-Q. Lu, D.-G. Yu, C.-J. Zhu and W.-J. Xiao, Visible light-driven organic photochemical synthesis in China, Sci. China: Chem., 2018, 62, 24-57.

25 G.-H. Li, Q.-Q. Han, Y.-Y. Sun, D.-M. Chen, Z.-L. Wang, X.-M. Xu and X.-Y. Yu, Visible-light induced cascade radical cyclization of sulfinic acids and o-(allyloxy)arylaldehydes towards functionalized chroman-4-ones, Chin. Chem. Lett., 2020, DOI: 10.1016/j.cclet.2020.03.007.

26 D. Q. Dong, L. X. Li, G. H. Li, Q. Deng, Z. L. Wang and S. Long, Visible-light-induced deoxygenative C2-sulfonylation of quinoline N-oxides with sulfinic acids for the synthesis of 2-sulfonylquinoline via radical reactions, Chin. J. Catal., 2019, 40, 1494-1498.

27 J. Hu, J. Wang, T. H. Nguyen and N. Zheng, The chemistry of amine radical cations produced by visible light photoredox catalysis, Beilstein J. Org. Chem., 2013, 9, 1977-2001.

28 A. Banerjee, Z. Lei and M. Y. Ngai, Acyl Radical Chemistry via Visible-Light Photoredox Catalysis, Synthesis, 2019, 303-333.

29 S. Yang, L. Wang, L. Wang and H. Li, Visible-Light Photoredox-Catalyzed Regioselective Sulfonylation of Alkenes Assisted by Oximes via [1,5]-H Migration, J. Org. Chem., 2020, 85, 564-573.

30 Q. Liu and L.-Z. Wu, Recent advances in visible-lightdriven organic reactions, Natl. Sci. Rev., 2017, 4, 359-380.

31 X.-X. Meng, Q.-Q. Kang, J.-Y. Zhang, Q. Li, W.-T. Wei and W.-M. He, Visible-light-initiated regioselective sulfonylation/cyclization of 1,6-enynes under photocatalyst- and additive-free conditions, Green Chem., 2020, 22, 13881392.

32 L.-Y. Xie, T.-G. Fang, J.-X. Tan, B. Zhang, Z. Cao, L.-H. Yang and W.-M. He, Visible-light-induced deoxygenative $\mathrm{C}_{2}$-sulfonylation of quinoline N-oxides with sulfinic acids, Green Chem., 2019, 21, 3858-3863.

33 S. Peng, Y. Lin and W. He, Visible Light-Induced Aldehyde Reductive Minisci Reaction towards N-Heterocycles, Chin. J. Org. Chem., 2020, 40, 541-542.

34 D. A. Nicewicz and D. W. C. MacMillan, Merging Photoredox Catalysis with Organocatalysis: The Direct Asymmetric Alkylation of Aldehydes, Science, 2008, 322, 77-80.

35 M. A. Ischay, M. E. Anzovino, J. Du and T. P. Yoon, Efficient Visible Light Photocatalysis of [2+2] Enone
Cycloadditions, J. Am. Chem. Soc., 2008, 130, 1288612887.

36 Z. Gu, H. Zhang, P. Xu, Y. Cheng and C. Zhu, VisibleLight-Induced Radical Tandem Aryldifluoroacetylation of Cinnamamides: Access to Difluoroacetylated Quinolone-2ones And 1-Azaspiro[4.5]decanes, Adv. Synth. Catal., 2015, 357, 3057-3063.

37 W. Fu, M. Zhu, G. Zou, C. Xu and Z. Wang, Visible-LightMediated Radical Aryldifluoroacetylation ofN-Arylacrylamides to give Difluoroacetylated Oxindoles, Asian J. Org. Chem., 2014, 3, 1273-1276.

38 X. Sun and S. Yu, Visible-light-promoted iminyl radical formation from vinyl azides: synthesis of 6-(fluoro)alkylated phenanthridines, Chem. Commun., 2016, 52, 1089810901.

39 S. Tang, Y. L. Deng, J. Li, W. X. Wang, G. L. Ding, M. W. Wang, Z. P. Xiao, Y. C. Wang and R. L. Sheng, Synthesis of Perfluorinated Isoquinolinediones through Visible-Light-Induced Cyclization of Alkenes, J. Org. Chem., 2015, 80, 12599-12605.

40 W. Fu, M. Zhu, G. Zou, C. Xu, Z. Wang and B. Ji, Visiblelight-mediated radical aryldifluoroacetylation of alkynes with ethyl bromodifluoroacetate for the synthesis of 3-difluoroacetylated coumarins, J. Org. Chem., 2015, 80, 4766-4770.

41 Z. Zhang, X. J. Tang and W. R. Dolbier Jr., PhotoredoxCatalyzed Intramolecular Difluoromethylation of N-Benzylacrylamides Coupled with a Dearomatizing Spirocyclization: Access to CF2H-Containing 2-Azaspiro [4.5]deca-6,9-diene-3,8-diones, Org. Lett., 2016, 18, 10481051.

42 H. G. Huang, M. L. Yu, X. L. Su, P. Guo, J. Zhao, J. B. Zhou and Y. Li, Sustainable Radical Cascades to Synthesize Difluoroalkylated Pyrrolo 1,2-a indoles, J. Org. Chem., 2018, 83, 2425-2437.

43 Q. Deng, L. Tan, Y. Xu, P. Liu and P. Sun, Synthesis of 6-Fluoroalkyl 6 H-Benzo[c]chromenes via Visible-LightPromoted Radical Addition/Cyclization of Biaryl Vinyl Ethers, J. Org. Chem., 2018, 83, 6151-6161.

44 T. Zhang, B. Chen, W. Wang, Q. Zhang, P. Wang, W. Wan, H. Deng, J. Hao and H. Jiang, Copper-Promoted Aryldifluoromethylenation of N-Arylacrylamides to 3-Benzo-diazolyldifluoromethylene-Substituted 2-Oxindoles, Asian J. Org. Chem., 2019, 8, 671-674.

45 J. L. Hu, T. J. Pu, Z. W. Xu, W. Y. Xu and Y. S. Feng, Cadmium Sulfide Quantum-Dot-Photocatalyzed Cascade Cyclization of Functionalized Difluoromethyl Chlorides with Unactivated Olefins, Adv. Synth. Catal., 2019, 361, 708-713.

46 H. Xiang, Q. L. Zhao, P. J. Xia, J. A. Xiao, Z. P. Ye, X. Xie, H. Sheng, X. Q. Chen and H. Yang, Visible-Light-Induced External Radical-Triggered Annulation To Access $\mathrm{CF}_{2^{-}}$ Containing Benzoxepine Derivatives, Org. Lett., 2018, 20, 1363-1366.

47 X. Liu, Z. Wu, Z. Zhang, P. Liu and P. Sun, Synthesis of trifluoroalkyl or difluoroalkyl phenanthridine derivatives via 
cascade reaction using an intramolecular cyano group as a radical acceptor under photoredox catalysis, Org. Biomol. Chem., 2018, 16, 414-423.

48 F. Ding, Y. Fang, Y. Jiang, K. Lin and L. Shi, Tandem Radical Cyclization for the Construction of DifluoroContaining Oxindoles and Quinoline-2,4-diones, Chem.Asian J., 2018, 13, 636-640.

49 T. C. McKee, H. R. Bokesch, J. L. McCormick, M. A. Rashid, D. Spielvogel, K. R. Gustafson, M. M. Alavanja, J. H. Cardellina and M. R. Boyd, Isolation and Characterization of New Anti-HIV and Cytotoxic Leads from Plants, Marine, and Microbial Organisms, J. Nat. Prod., 1997, 60, 431-438.

50 B. Lee, H. D. Basavarajappa, R. S. Sulaiman, X. Fei, S.-Y. Seo and T. W. Corson, The first synthesis of the antiangiogenic homoisoflavanone, cremastranone, Org. Biomol. Chem., 2014, 12, 7673-7677.

51 L. Xiong, H. Hu, C.-W. Wei and B. Yu, Radical Reactions for the Synthesis of 3-Substituted Chroman-4-ones, Eur. J. Org. Chem., 2020, 1588-1597.

52 N. N. Zhou, M. X. Wu, M. Zhang and X. Q. Zhou, VisibleLight-Induced Difluoroacetylation of O-(Allyloxy)ArylAldehydes: Access to Difluoroacetylated Chroman-4-ones, Asian J. Org. Chem., 2019, 8, 828-831.

53 X. Huang, Y. G. Zhang, C. S. Zhang, L. Zhang, Y. Xu, L. C. Kong, Z. X. Wang and B. Peng, The orthoDifluoroalkylation of Aryliodanes with Enol Silyl Ethers: Rearrangement Enabled by a Fluorine Effect, Angew. Chem., Int. Ed., 2019, 58, 5956-5961.

54 C. Yu, N. Iqbal, S. Park and E. J. Cho, Selective difluoroalkylation of alkenes by using visible light photoredox catalysis, Chem. Commun., 2014, 50, 12884-12887.

55 X. Li, S. Li, S. Sun, F. Yang, W. Zhu, Y. Zhu, Y. Wu and Y. Wu, Direct Decarboxylative Alkynylation of $\alpha, \alpha$-Difluoroarylacetic Acids under Transition MetalFree Conditions, Adv. Synth. Catal., 2016, 358, 16991704.

56 X.-J. Tang and W. R. Dolbier Jr., Efficient Cu-catalyzed Atom Transfer Radical Addition Reactions of Fluoroalkylsulfonyl Chlorides with Electron-deficient Alkenes Induced by Visible Light, Angew. Chem., Int. Ed., 2015, 54, 4246-4249.

57 M. Zhang, W. Li, Y. Duan, P. Xu, S. Zhang and C. Zhu, Cascade Photoredox/Iodide Catalysis: Access to Difluoro$\gamma$-lactams via Aminodifluoroalkylation of Alkenes, Org. Lett., 2016, 18, 3266-3269.

58 Y. L. Zhou, Z. M. Xiong, J. Y. Qiu, L. C. Kong and G. G. Zhu, Visible light photocatalytic acyldifluoroalkylation of unactivated alkenes for the direct synthesis of gem- difluorinated ketones, Org. Chem. Front., 2019, 6, 1022-1026.

59 Z. J. Shen, S. C. Wang, W. J. Hao, S. Z. Yang, S. J. Tu and B. Jiang, Switching between Copper-Catalysis and Photocatalysis for Tunable Halofluoroalkylation and Hydrofluoroalkylation of 1,6-Enynes toward 1-Indenones, Adv. Synth. Catal., 2019, 361, 3837-3851.
60 M. Li, C. T. Wang, Y. F. Qiu, X. Y. Zhu, Y. P. Han, Y. Xia, X. S. Li and Y. M. Liang, Base promoted direct difunctionalization/cascade cyclization of 1,6-enynes, Chem. Commun., 2018, 54, 5334-5337.

61 P. B. Zhang, C. Wang, M. C. Cui, M. S. Du, W. W. Li, Z. X. Jia and Q. Zhao, Synthesis of Difluoroalkylated Benzofuran, Benzothiophene, and Indole Derivatives via Palladium-Catalyzed Cascade Difluoroalkylation and Arylation of 1,6-Enynes, Org. Lett., 2020, 22, 1149-1154.

62 J. Wang, M. Sánchez-Roselló, J. L. Aceña, C. del Pozo, A. E. Sorochinsky, S. Fustero, V. A. Soloshonok and H. Liu, Fluorine in Pharmaceutical Industry: Fluorine-Containing Drugs Introduced to the Market in the Last Decade (2001-2011), Chem. Rev., 2014, 114, 2432-2506.

63 M. O. Anderson, J. Zhang, Y. Liu, C. Yao, P.-W. Phuan and A. S. Verkman, Nanomolar Potency and Metabolically Stable Inhibitors of Kidney Urea Transporter UT-B, J. Med. Chem., 2012, 55, 5942-5950.

64 M. Médebielle, Electrochemical addition of chlorodifluoroacetyl aromatic compounds to electron-rich olefinic substrates. A convenient synthesis of gem-difluoro heterocyclic compounds, Tetrahedron Lett., 1995, 36, 2071-2074.

65 P. Hapiot and M. Médebielle, Electrochemically induced free-radical tandem cyclisation of chlorodifluoromethylated ketones: Application to the synthesis of gem-difluorinated heterocycles, J. Fluorine Chem., 2001, 107, 285-300.

66 C. Adouama, R. Keyrouz, G. Pilet, C. Monnereau, D. Gueyrard, T. Noël and M. Médebielle, Access to cyclic gem-difluoroacyl scaffolds via electrochemical and visible light photocatalytic radical tandem cyclization of heteroaryl chlorodifluoromethyl ketones, Chem. Commun., 2017, 53, 5653-5656.

67 C. Qu, P. Xu, W. Ma, Y. Cheng and C. Zhu, A novel visible light mediated radical cyclization of enol lactones: a concise method for fluorinated polycyclic lactone scaffolds, Chem. Commun., 2015, 51, 13508-13510.

68 C. Qu, Z. Wu, W. Li, H. Du and C. Zhu, Electron Catalytic Photochemical Cascade Carbodifluoroalkylation/Radical Cyclization of Methylene-2-oxazolines, Adv. Synth. Catal., 2017, 359, 1672-1677.

69 P. Peng, G. Z. Huang, Y. X. Sun, X. Wang, J. J. Wu and F. $\mathrm{H}$. Wu, Copper-mediated cascade radical cyclization of olefins with naphthalenyl iododifluoromethyl ketones, Org. Biomol. Chem., 2019, 17, 6426-6431.

70 C. Liu, Y. J. Yang, J. Y. Dong, M. D. Zhou, L. Li and H. Wang, Copper/B(2)pin(2)-Catalyzed Difluoroalkylation of Methylenecyclopropanes with Bromodifluorinated Acetates and Acetamides: One-Pot Synthesis of $\mathrm{CF}_{2}{ }^{-}$ Containing Dihydronaphthalene Derivatives, J. Org. Chem., 2019, 84, 9937-9945.

71 X. Wang, J. Liu, Z. Yu, M. Guo, X. Tang and G. Wang, Desulfonylation-Initiated Distal Alkenyl Migration in Copper-Catalyzed Alkenylation of Unactivated Alkenes, Org. Lett., 2018, 20, 6516-6519.

$72 \mathrm{X}$. J. Wei and T. Noel, Visible-Light Photocatalytic Difluoroalkylation-Induced 1, 2-Heteroarene Migration of 
Allylic Alcohols in Batch and Flow, J. Org. Chem., 2018, 83, 11377-11384.

73 L. Hu, Q. Deng, Y. Zhou, X. Zhang and Y. Xiong, Cu2Ocatalyzed phosphonyldifluoromethylation of allylic alcohols through a radical 1,2-aryl migration, Tetrahedron, 2020, 76, 130949.

74 D. M. Whalley, H. A. Duong and M. F. Greaney, Alkene Carboarylation through Catalyst-Free, Visible LightMediated Smiles Rearrangement, Chem. - Eur. J., 2019, 25, 1927-1930.

75 J. Liu, W. P. Li, J. Xie and C. J. Zhu, Photoredox 1,2dicarbofunctionalization of unactivated alkenes via tandem radical difluoroalkylation and alkynyl migration, Org. Chem. Front., 2018, 5, 797-800.

76 M. Li, X. Y. Zhu, Y. F. Qiu, Y. P. Han, Y. Xia, C. T. Wang, X. S. Li, W. X. Wei and Y. M. Liang, Metal-Free Promoted CF2/CF3-Difunctionalization of Unactivated Alkenes, Adv. Synth. Catal., 2019, 361, 2945-2950.

77 W. W. Jin, M. C. Wu, Z. M. Xiong and G. G. Zhu, Visiblelight induced three-component alkynyl-difluoroalkylation of unactivated alkenes, Chem. Commun., 2018, 54, 79247927.

$78 \mathrm{~J}$. Ye and S. Ma, Conquering three-carbon axial chirality of allenes, Org. Chem. Front., 2014, 1, 1210-1224.

79 (a) J. Ye and S. Ma, Palladium-Catalyzed Cyclization Reactions of Allenes in the Presence of Unsaturated Carbon-Carbon Bonds, Acc. Chem. Res., 2014, 47, 9891000; (b) J.-W. Gu, Q.-Q. Min, L.-C. Yu and X. Zhang, Tandem Difluoroalkylation-Arylation of Enamides Catalyzed by Nickel, Angew. Chem., Int. Ed., 2016, 55, 12270-12274.

80 K.-F. Zhang, K.-J. Bian, C. Li, J. Sheng, Y. Li and X.-S. Wang, Nickel-Catalyzed Carbofluoroalkylation of 1,3Enynes to Access Structurally Diverse Fluoroalkylated Allenes, Angew. Chem., Int. Ed., 2019, 58, 5069-5074.

81 X. L. Lv, C. Wang, Q. L. Wang and W. Shu, Rapid Synthesis of gamma-Arylated Carbonyls Enabled by the Merge of Copper- and Photocatalytic Radical Relay Alkylarylation of Alkenes, Org. Lett., 2019, 21, 56-59.

82 J. Cao, G. Wang, L. Gao, H. Chen, X. Liu, X. Cheng and S. Li, Perfluoroalkylative pyridylation of alkenes via 4-cyanopyridine-boryl radicals, Chem. Sci., 2019, 10, 2767-2772.

83 X. G. Wang, Y. Li, H. C. Liu, B. S. Zhang, X. Y. Gou, Q. Wang, J. W. Ma and Y. M. Liang, Three-Component Ruthenium-Catalyzed Direct Meta-Selective C-H Activation of Arenes: A New Approach to the Alkylarylation of Alkenes, J. Am. Chem. Soc., 2019, 141, 13914-13922.

84 X. Yong, Y. F. Han, Y. Li, R. J. Song and J. H. Li, Alkylarylation of styrenes via direct $\mathrm{C}(\mathrm{sp}(3))-\mathrm{Br} / \mathrm{C}(\mathrm{sp}(2))-\mathrm{H}$ functionalization mediated by photoredox and copper cooperative catalysis, Chem. Commun., 2018, 54, 1281612819.

85 D. Zheng and A. Studer, Asymmetric Synthesis of Heterocyclic gamma-Amino-Acid and Diamine Derivatives by Three-Component Radical Cascade Reactions, Angew. Chem., Int. Ed., 2019, 58, 15803-15807.
86 J.-L. Li, Y.-Q. Liu, W.-L. Zou, R. Zeng, X. Zhang, Y. Liu, B. Han, Y. He, H.-J. Leng and Q.-Z. Li, Radical Acylfluoroalkylation of Olefins through N-Heterocyclic Carbene Organocatalysis, Angew. Chem., Int. Ed., 2020, 59, 1863-1870.

87 W. Shu, E. Merino and C. Nevado, Visible Light Mediated, Redox Neutral Remote 1,6-Difunctionalizations of Alkenes, ACS Catal., 2018, 8, 6401-6406.

88 J. Zhang, W. Jin, C. Cheng and F. Luo, Copper-catalyzed remote oxidation of alcohols initiated by radical difluoroalkylation of alkenes: facile access to difluoroalkylated carbonyl compounds, Org. Biomol. Chem., 2018, 16, 38763880 .

89 L. Li, H. Luo, Z. Zhao, Y. Li, Q. Zhou, J. Xu, J. Li and Y. N. Ma, Photoredox-Catalyzed Remote Difunctionalizations of Alkenes To Synthesize Fluoroalkyl Ketones with Dimethyl Sulfoxide as the Oxidant, Org. Lett., 2019, 21, 9228-9231.

90 G.-T. Song, C.-H. Qu, J.-P. Meng, Z.-G. Xu, C.-H. Zhou and Z.-Z. Chen, Photoredox catalytic cascade radical addition/ aromatization of methylene-2-oxazolines: Mild access to C(sp3)-difluoro-oxazole derivatives, Tetrahedron Lett., 2019, 60, 151246.

91 T. Brandhofer and O. G. Mancheño, Versatile RuPhotoredox-Catalyzed Functionalization of DehydroAmino Acids and Peptides, ChemCatChem, 2019, 11, 3797-3801.

92 A. Correa and R. Martín, Metal-Catalyzed Carboxylation of Organometallic Reagents with Carbon Dioxide, Angew. Chem., Int. Ed., 2009, 48, 6201-6204.

93 M. Börjesson, T. Moragas, D. Gallego and R. Martin, Metal-Catalyzed Carboxylation of Organic (Pseudo)halides with CO2, ACS Catal., 2016, 6, 6739-6749.

94 S. Wang, G. Du and C. Xi, Copper-catalyzed carboxylation reactions using carbon dioxide, Org. Biomol. Chem., 2016, 14, 3666-3676.

95 Q. Zhu, L. Wang, C. Xia and C. Liu, Recent Advance of Transition Metal-Catalyzed Direct C-H Bond Carboxylation with $\mathrm{CO}_{2}$, Chin. J. Org. Chem., 2016, 36, 2813-2821.

96 S. Zhang, X. Li and L.-N. He, Reductive Carboxylation of Unsaturated Hydrocarbons with Carbon Dioxide, Acta Chim. Sin., 2016, 74, 17-23.

97 W. Zhang, C. Guo and X. Lü, Ni-catalyzed direct carboxylation of unactivated alkyl electrophiles with carbon dioxide, Chin. J. Catal., 2016, 37, 215-217.

98 Y.-G. Chen, X.-T. Xu, K. Zhang, Y.-Q. Li, L.-P. Zhang, P. Fang and T.-S. Mei, Transition-Metal-Catalyzed Carboxylation of Organic Halides and Their Surrogates with Carbon Dioxide, Synthesis, 2018, 35-48.

99 X.-F. Wu and F. Zheng, Synthesis of Carboxylic Acids and Esters from $\mathrm{CO}_{2}$, Top. Curr. Chem., 2017, 375, 4.

100 Z. B. Yin, J. H. Ye, W. J. Zhou, Y. H. Zhang, L. Ding, Y. Y. Gui, S. S. Yan, J. Li and D. G. Yu, OxyDifluoroalkylation of Allylamines with $\mathrm{CO} 2$ via VisibleLight Photoredox Catalysis, Org. Lett., 2018, 20, 190-193. 
101 F. Xiao, F. Wu, X. Yang, Y. Shen and X. Shi, A convenient synthesis of fluoroalkylated $\gamma$-butyrolactones from polyfluoroalkyl iodides and 4-pentenoic acid catalyzed by $\mathrm{Pd}\left(\mathrm{PPh}_{3}\right)_{4}$, J. Fluorine Chem., 2005, 126, 319-323.

102 W. Sha, W. Zhang, S. Ni, H. Mei, J. Han and Y. Pan, Photoredox-Catalyzed Cascade Difluoroalkylation and Intramolecular Cyclization for Construction of Fluorinated $\gamma$-Butyrolactones, J. Org. Chem., 2017, 82, 9824-9831.

103 Y. Da, S. Han, X. Du, S. Liu, L. Liu and J. Li, Copper(I)Catalyzed Oxydifluoroalkylation of Alkenes: A Route to Functionalization of Lactones, Org. Lett., 2018, 20, 51495152.

104 F. Y. Yuan, S. Zhou, Y. Y. Yang, M. J. Guo, X. Y. Tang and G. W. Wang, Copper catalyzed one-pot difluoroalkylation and lactonization of unsaturated carboxylic acids, Org. Chem. Front., 2018, 5, 3306-3309.

105 Q. Yang, Q. Q. Lin, H. Y. Xing and Z. G. Zhao, Visiblelight-mediated difluoromethylphosphonation of alkenes for the synthesis of CF2P-containing heterocycles, Org. Chem. Front., 2019, 6, 3939-3943.

106 Y. Yang, F. Yuan, X. Ren, G. Wang, W. Zhao, X. Tang and M. Guo, Copper-Catalyzed Oxydifluoroalkylation of Hydroxyl-Containing Alkenes, J. Org. Chem., 2019, 84, 4507-4516.

107 X. Wang, M. Li, Y. Yang, M. Guo, X. Tang and G. Wang, One-pot Construction of Difluorinated Pyrrolizidine and Indolizidine Scaffolds via Copper-Catalyzed Radical Cascade Annulation, Adv. Synth. Catal., 2018, 360, 21512156.

108 J. Liao, L. Ouyang, Y. Lai and R. Luo, PhotoredoxCatalyzed Oxy-/Aminofluoroalkylative Cyclization of Alkenes, J. Org. Chem., 2020, 85, 5590-5597.

109 J. J. Yu, D. P. Wang, Y. Xu, Z. Wu and C. Zhu, Distal Functional Group Migration for Visible-light Induced Carbo-difluoroalkylation/monofluoroalkylation of Unactivated Alkenes, Adv. Synth. Catal., 2018, 360, 744750.

110 W. G. Kong, C. J. Yu, H. J. An and Q. L. Song, CopperCatalyzed Intermolecular Reductive Radical Difluoroalkylation-Thiolation of Aryl Alkenes, Org. Lett., 2018, 20, 4975-4978.

111 Y. Chen, L. Li, Y. Ma and Z. Li, Cobalt-Catalyzed ThreeComponent Difluoroalkylation-Peroxidation of Alkenes, J. Org. Chem., 2019, 84, 5328-5338.

112 R. Xu and C. Cai, Three- component difluoroalkylationthiolation of alkenes by iron- facilitated visible- light photoredox catalysis, Chem. Commun., 2019, 55, 43834386.

113 K. S. Hagen, J. G. Reynolds and R. H. Holm, Definition of reaction sequences resulting in self-assembly of $\left[\mathrm{Fe}_{4} \mathrm{~S}_{4}(\mathrm{SR})_{4}\right]_{2}$ - clusters from simple reactants, J. Am. Chem. Soc., 1981, 103, 4054-4063.

114 R. Xu and C. Cai, Three-component difluoroalkylamination of alkenes mediated by photoredox and iron cooperative catalysis, Org. Biomol. Chem., 2019, 17, 8541-8545.
115 Y. Murata, T. Shimada and T. Nishikata, Radical and Cation Crossover Reaction System Enables Synthesis of Complex Aliphatic Chains Possessing Functionalized Quaternary Carbons, Bull. Chem. Soc. Jpn., 2019, 92, 14191429.

116 G. H. Li, D. Q. Dong, Q. Deng, S. Q. Yan and Z. L. Wang, Copper-Catalyzed Deoxygenative C2-Sulfonylation of Quinoline N-Oxides with DABSO and Phenyldiazonium Tetrafluoroborates for the Synthesis of 2-Sulfonylquinolines via a Radical Reaction, Synthesis, 2019, 3313-3319.

117 G. Qiu, L. Lai, J. Cheng and J. Wu, Recent advances in the sulfonylation of alkenes with the insertion of sulfur dioxide via radical reactions, Chem. Commun., 2018, 54, 10405-10414.

118 S. Ye, G. Qiu and J. Wu, Inorganic sulfites as the sulfur dioxide surrogates in sulfonylation reactions, Chem. Commun., 2019, 55, 1013-1019.

119 Y. Liu, Q. Lin, Z. Xiao, C. Zheng, Y. Guo, Q. Y. Chen and C. Liu, Zinc-Mediated Intermolecular Reductive Radical Fluoroalkylsulfination of Unsaturated Carbon-Carbon Bonds with Fluoroalkyl Bromides and Sulfur Dioxide, Chemistry, 2019, 25, 1824-1828.

120 C. Xu, Z.-F. Yang, L. An and X. Zhang, Nickel-Catalyzed Difluoroalkylation-Alkylation of Enamides, ACS Catal., 2019, 9, 8224-8229.

121 B. Zhao, Z. Li, Y. Wu, Y. Wang, J. Qian, Y. Yuan and Z. Shi, An Olefinic 1,2-Boryl-Migration Enabled by Radical Addition: Construction of gem-Bis(boryl)alkanes, Angew. Chem., Int. Ed., 2019, 58, 9448-9452.

122 D. K. Li, T. T. Mao, J. B. Huang and Q. Zhu, CopperCatalyzed Bromodifluoroacetylation of Alkenes with Ethyl Bromodifluoroacetate, J. Org. Chem., 2018, 83, 1044510452.

123 L. Zhao, Y. Huang, Z. Wang, E. Zhu, T. Mao, J. Jia, J. W. Gu, X. F. Li and C. Y. He, OrganophosphineCatalyzed Difluoroalkylation of Alkenes, Org. Lett., 2019, 21, 6705-6709.

124 J. Sheng, K. J. Bian, Y. M. Su, G. X. Liao, R. M. Duan, C. Li, Z. H. Liu and X. S. Wang, Visible light-mediated atom transfer radical addition to styrene: base controlled selective (phenylsulfonyl)difluoromethylation, Org. Chem. Front., 2020, 7, 617-621.

125 L. X. Li, Y. N. Ma, M. Tang, J. Guo, Z. Yang, Y. Z. Yan, X. T. Ma and L. Tang, Photoredox-Catalyzed Oxydifluoroalkylation of Styrenes for Access to Difluorinated Ketones with DMSO as an Oxidant, $A d v$. Synth. Catal., 2019, 361, 3723-3728.

126 S. Tian, X. Song, D. Zhu and M. Wang, Alternative Palladium-Catalyzed Vinylic $\mathrm{C}-\mathrm{H}$ Difluoroalkylation of Ketene Dithioacetals Using Bromodifluoroacetate Derivatives, Adv. Synth. Catal., 2018, 360, 1414-1419.

127 Y. N. Zhao, Y. C. Luo, Z. Y. Wang and P. F. Xu, A new approach to access difluoroalkylated diarylmethanes via visible-light photocatalytic cross-coupling reactions, Chem. Commun., 2018, 54, 3993-3996. 
128 J. M. Muñoz-Molina and P. J. Perez, Ruthenium-Catalyzed Heck-Type Alkenylation of Alkyl Bromides, J. Org. Chem., 2019, 84, 8289-8296.

129 M. Uno, S. Sumino, T. Fukuyama, M. Matsuura, Y. Kuroki, Y. Kishikawa and I. Ryu, Synthesis of 4,4-Difluoroalkenes by Coupling of alpha-Substituted alpha,alpha-Difluoromethyl Halides with Allyl Sulfones under Photoredox Catalyzed Conditions, J. Org. Chem., 2019, 84, 9330-9338.

130 X. Li, R. Zhang, X. Zhang, P. Zhu and T. Yao, SilverCatalyzed Decarboxylative Allylation of Difluoroarylacetic Acids with Allyl Sulfones in Water, Chem.-Asian J., 2020, 15, 1175-1179.

131 Y. W. Sun and G. Z. Zhang, Photoinduced Decarboxylative Amino-Fluoroalkylation of Maleic Anhydride, Chem. - Eur. J., 2020, 26, 419-422.

132 E. Sekino, T. Kumamoto, T. Tanaka, T. Ikeda and T. Ishikawa, Concise Synthesis of Anti-HIV-1 Active (+)-Inophyllum B and (+)-Calanolide A by Application of (-)-Quinine-Catalyzed Intramolecular Oxo-Michael Addition, J. Org. Chem., 2004, 69, 2760-2767.

133 P. Anand, B. Singh and N. Singh, A review on coumarins as acetylcholinesterase inhibitors for Alzheimer's disease, Bioorg. Med. Chem., 2012, 20, 1175-1180.

134 A. M. Musiliyu, S. C. John and M. O. F. Khan, A Review of Coumarin Derivatives in Pharmacotherapy of Breast Cancer, Curr. Med. Chem., 2008, 15, 2664-2679.

135 S. H. Hao, L. X. Li, D. Q. Dong, Z. L. Wang and X. Y. Yu, Synthesis of coumarins derivatives via decarboxylative cross-coupling of coumarin-3-carboxylic acid with benzylic C(sp(3))-H bond, Tetrahedron Lett., 2018, 59, 4073-4075.

136 D. Song, C. M. Wang, Z. P. Ye, P. J. Xia, Z. X. Deng, J. A. Xiao, H. Y. Xiang and H. Yang, Visible-Light-Driven, Photoredox-Catalyzed Cascade of ortho-Hydroxycinnamic Esters To Access 3-Fluoroalkylated Coumarins, J. Org. Chem., 2019, 84, 7480-7487.

137 S. B. Nagode, A. K. Chaturvedi and N. Rastogi, Visiblelight-catalyzed Tandem DifluoroacetylationIntramolecular Cyclization of 1,3-Diarylpropynones: Access to Difluoroacetylated Indenones, Asian J. Org. Chem., 2017, 6, 453-457.

138 T. T. Zhang, M. J. Luo, F. Teng, Y. Li, M. Hu and J. H. Li, Photoredox Alkylarylation of N -Benzyl- N -(2-ethynylaryl)Amides with $\alpha$-Bromoalkyl Esters: Access to Dibenzazepines, Adv. Synth. Catal., 2019, 361, 4645-4650.

139 D. Liu, M. J. Jiao, X. Z. Wang and P. F. Xu, Metal-Free Visible-Light-Induced Construction of DifluoroContaining Dibenzazepines, Org. Lett., 2019, 21, 47454749.

140 Y. Zhang, J. Zhang, B. Hu, M. Ji, S. Ye and G. Zhu, Synthesis of Difluoromethylated and Phosphorated Spiro [5.5]trienones via Dearomative Spirocyclization of Biaryl Ynones, Org. Lett., 2018, 20, 2988-2992.

141 S. Han, S. Liu, L. Liu, L. Ackermann and J. Li, CobaltCatalyzed Diastereoselective Difluoroalkylation/Giese Addition Domino Reactions, Org. Lett., 2019, 21, 53875391.
142 D. Y. Qian and J. L. Zhang, Gold-catalyzed cyclopropanation reactions using a carbenoid precursor toolbox, Chem. Soc. Rev., 2015, 44, 677-698.

143 D. M. Patterson, L. A. Nazarova, B. Xie, D. N. Kamber and J. A. Prescher, Functionalized Cyclopropenes As Bioorthogonal Chemical Reporters, J. Am. Chem. Soc., 2012, 134, 18638-18643.

144 Y. Y. Yang, P. Antoni, M. Zimmer, K. Sekine, F. F. Mulks, L. Hu, L. M. Zhang, M. Rudolph, F. Rominger and A. S. K. Hashmi, Dual Gold/Silver Catalysis Involving Alkynylgold(III) Intermediates Formed by Oxidative Addition and Silver-Catalyzed C-H Activation for the Direct Alkynylation of Cyclopropenes, Angew. Chem., Int. Ed., 2019, 58, 5129-5133.

145 K. Chen, X. Y. Huang, S. B. J. Kan, R. K. Zhang and F. H. Arnold, Enzymatic construction of highly strained carbocycles, Science, 2018, 360, 71-75.

146 Z.-Q. Zhang, M.-M. Zheng, X.-S. Xue, I. Marek, F.-G. Zhangand J.-A. Ma, Catalytic Enantioselective Cyclopropenation of Internal Alkynes: Access to Difluoromethylated ThreeMembered Carbocycles, Angew. Chem., Int. Ed., 2019, 58, 18191-18196.

147 X. Feng, X. Wang, H. Chen, X. Tang, M. Guo, W. Zhao and G. Wang, Copper-mediated regioselective hydrodifluoroalkylation of alkynes, Org. Biomol. Chem., 2018, 16, 28412845.

148 W. Q. Fu and Q. Song, Copper-Catalyzed Radical Difluoroalkylation and Redox Annulation of Nitroalkynes for the Construction of C2-Tetrasubstituted Indolin-3ones, Org. Lett., 2018, 20, 393-396.

149 J. Xu, F. Zhang, S. Zhang, L. Zhang, X. Yu, J. Yan and Q. Song, Radical Promoted C(sp(2))-S Formation and C(sp (3))-S Bond Cleavage: Access to 2-Substituted Thiochromones, Org. Lett., 2019, 21, 1112-1115.

150 Y. Zhang, S. Ye, M. Ji, L. Li, D. Guo and G. Zhu, CopperCatalyzed Radical Cascade Difluoromethylation/ Cyclization of 2-(3-Arylpropioloyl)benzaldehydes: A Route to Difluoromethylated Naphthoquinones, J. Org. Chem., 2017, 82, 6811-6818.

151 H.-L. Hua, B.-S. Zhang, Y.-T. He, Y.-F. Qiu, J.-Y. Hu, Y.-C. Yang and Y.-M. Liang, Copper-catalyzed difluoromethylation of propargylamide-substituted indoles: synthesis of mono- and bis-difluoromethylated indoloazepinone derivatives, Chem. Commun., 2016, 52, 10396-10399.

152 J. W. Ma, Q. Wang, X. G. Wang and Y. M. Liang, Palladium-Catalyzed Cascade Difluoroalkylation/ Cyclization of N-Propargylamides: Synthesis of Oxazoles and Oxazolines, J. Org. Chem., 2018, 83, 13296-13307.

153 G. Wu and A. Jacobi von Wangelin, Stereoselective cobaltcatalyzed halofluoroalkylation of alkynes, Chem. Sci., 2018, 9, 1795-1802.

154 (a) S. F. Wang, J. Zhang, L. C. Kong, Z. Tan, Y. H. Bai and G. G. Zhu, Palladium-Catalyzed anti-Selective Fluoroalkylboration of Internal and Terminal Alkynes, Org. Lett., 2018, 20, 5631-5635; (b) W.-H. Guo, H.-Y. Zhao, Z.-J. Luo, S. Zhang and X. Zhang, Fluoroalkylation- 
Borylation of Alkynes: An Efficient Method To Obtain (Z) Tri- and Tetrasubstituted Fluoroalkylated Alkenylboronates, ACS Catal., 2019, 9, 38-43.

155 B. S. Zhang, L. Y. Gao, Z. Zhang, Y. H. Wen and Y. M. Liang, Three-component difluoroalkylation and trifluoromethylthiolation/trifluoromethylselenolation of pi-bonds, Chem. Commun., 2018, 54, 1185-1188.

156 T. Mao, M. J. Ma, L. Zhao, D. P. Xue, Y. B. Yu, J. W. Gu and C. Y. He, A general and green fluoroalkylation reaction promoted via noncovalent interactions between acetone and fluoroalkyl iodides, Chem. Commun., 2020, 56, 1815-1818.

157 N. Iqbal, N. Iqbal, S. S. Han and E. J. Cho, Synthesis of fluoroalkylated alkynes via visible-light photocatalysis, Org. Biomol. Chem., 2019, 17, 1758-1762.

158 X. Wang, J. Hu, J. Ren, T. Wu, J. Wu and F. Wu, Palladium-catalyzed one-pot construction of difluorinated 1,3-enynes from $\alpha, \alpha, \alpha$-iododifluoroacetones and alkynes, Tetrahedron, 2019, 75, 130715.

159 G. Tu, C. Yuan, Y. Li, J. Zhang and Y. Zhao, A LigandEnabled Palladium-Catalyzed Highly para-Selective Difluoromethylation of Aromatic Ketones, Angew. Chem., Int. Ed., 2018, 57, 15597-15601.

160 Y. J. Mao, B. X. Wang, Q. Z. Wu, K. Zhou, S. J. Lou and D. Q. Xu, Pd-Catalyzed para-selective C-H difluoromethylation of aromatic carbonyls, Chem. Commun., 2019, 55, 2019-2022.

161 J. Zhou, F. Wang, Z. Lin, C. Cheng, Q. Zhang and J. Li, Visible-Light-Induced para-Selective $\mathrm{C}(\mathrm{sp}(2))-\mathrm{H}$ Difluoroalkylation of Diverse (Hetero)aromatic Carbonyls, Org. Lett., 2020, 22, 68-72.

162 W. K. Tang, F. Tang, J. Xu, Q. Zhang, J. J. Dai, Y. S. Feng and H. J. Xu, Photocatalytic site-selective C-H difluoroalkylation of aromatic aldehydes, Chem. Commun., 2020, 56, 1497-1500.

163 C. Yuan, L. Zhu, R. Zeng, Y. Lan and Y. Zhao, Ruthenium (II)-Catalyzed C-H Difluoromethylation of Ketoximes: Tuning the Regioselectivity from the meta to the para Position, Angew. Chem., Int. Ed., 2018, 57, 1277-1281.

164 P. Gandeepan, J. Koeller, K. Korvorapun, J. Mohr and L. Ackermann, Visible-Light-Enabled RutheniumCatalyzed meta-C-H Alkylation at Room Temperature, Angew. Chem., Int. Ed., 2019, 58, 9820-9825.

165 C. Jia, S. Wang, X. Lv, G. Li, L. Zhong, L. Zou and X. Cui, Ruthenium-Catalyzed meta-CAr-H Bond Difluoroalkylation of 2-Phenoxypyridines, Eur. J. Org. Chem., 2020, 1992-1995.

166 S. Cai, Y. Tian, J. Zhang, Z. Liu, M. Lu, W. Weng and M. Huang, Carbotrifluoromethylation of Allylic Alcohols via 1,2-Aryl Migration Promoted by Visible-Light-Induced Photoredox Catalysis, Adv. Synth. Catal., 2018, 360, 4084-4088.

167 X. Wang, S. Zhao, J. Liu, D. Zhu, M. Guo, X. Tang and G. Wang, Copper-Catalyzed C-H Difluoroalkylations and Perfluoroalkylations of Alkenes and (Hetero)arenes, Org. Lett., 2017, 19, 4187-4190.

168 Q. Lin, L. Chu and F.-L. Qing, Direct Introduction of Ethoxycarbonyldifluoromethyl-Group to Heteroarenes with Ethyl Bromodifluoroacetate via Visible-Light Photocatalysis, Chin. J. Chem., 2013, 31, 885-891.

169 S. Y. Yan, Z. Z. Zhang, Y. H. Liu, G. Liao, P. X. Li and B. F. Shi, Copper-Catalyzed C-H Ethoxycarbonyldifluoromethylation of Indoles and Pyrroles, Asian J. Org. Chem., 2018, 7, 1319-1322.

170 (a) V. Soni, D. M. Sharma and B. Punji, Nickel-Catalyzed Regioselective C(2)-H Difluoroalkylation of Indoles with Difluoroalkyl Bromides, Chem. - Asian J., 2018, 13, 25162521; (b) L. Ackermann, B. Punji and W. Song, User-Friendly [(Diglyme) $\left.\mathrm{NiBr}_{2}\right]$-Catalyzed Direct Alkylations of Heteroarenes with Unactivated Alkyl Halides through $\mathrm{C}=\mathrm{H}$ Bond Cleavages, Adv. Synth. Catal., 2011, 353, 3325-3329.

171 C. Bottecchia, R. Martín, I. Abdiaj, E. Crovini, J. Alcazar, J. Orduna, M. J. Blesa, J. R. Carrillo, P. Prieto and T. Noël, De novo Design of Organic Photocatalysts: Bithiophene Derivatives for the Visible-light Induced $\mathrm{C}-\mathrm{H}$ Functionalization of Heteroarenes, Adv. Synth. Catal., 2019, 361, 945-950.

172 X. Chen, W. Xu, K. Wang, M. Mo, W. Zhang, L. Du, X. Yuan, Y. Xu, Y. Wang and J. Shen, Discovery of a Novel Series of Imidazo[1,2-a]pyrimidine Derivatives as Potent and Orally Bioavailable Lipoprotein-Associated Phospholipase A2 Inhibitors, J. Med. Chem., 2015, 58, 8529-8541.

173 E.-G. Cecile and G. Alain, Recent Progress in the Pharmacology of Imidazo[1,2-a]pyridines, Mini-Rev. Med. Chem., 2007, 7, 888-899.

174 S. Mishra, P. Mondal, M. Ghosh, S. Mondal and A. Hajra, Copper-catalyzed $\mathrm{C}-\mathrm{H}$ ethoxycarbonyldifluoromethylation of imidazoheterocycles, Org. Biomol. Chem., 2016, 14, 1432-1436.

175 G. Yin, M. Zhu and W. Fu, Visible-light-induced photocatalytic difluoroacetylation of imidazopyridines via direct and regioselective $\mathrm{C} \mathrm{H}$ functionalization, J. Fluorine Chem., 2017, 199, 14-19.

176 M. Q. Hua, F. Y. Mou, N. H. Wang, Y. Chen, H. Y. Xiong, Y. Huang, C. L. Lei and Q. Zhang, Visible-light-induced photocatalytic difluoroalkylation of 3-substituted benzo d isoxazoles via direct and regioselective $\mathrm{C}-\mathrm{H}$ functionalization, Tetrahedron Lett., 2018, 59, 4449-4453.

177 M. Singsardar, S. Mondal, S. Laru and A. Hajra, Organophotoredox-Catalyzed $\mathrm{C}(\mathrm{sp}(2))-\mathrm{H}$ Difluoromethylenephosphonation of Imidazoheterocycles, Org. Lett., 2019, 21, 5606-5610.

178 C. H. Qu, G. T. Song, J. Xu, W. Yan, C. H. Zhou, H. Y. Li, Z. Z. Chen and Z. G. Xu, Merging Visible Light with CrossCoupling: The Photochemical Direct C-H Difluoroalkylation of Imidazopyridines, Org. Lett., 2019, 21, 8169-8173.

179 S. Han, A. Liang, X. Ren, X. Gao, J. Li, D. Zou, Y. Wu and Y. Wu, Copper-catalyzed remote $\mathrm{CH}$ ethoxycarbonyldifluoromethylation of 8-aminoquinolines with bis(pinacolato) diboron as reductant, Tetrahedron Lett., 2017, 58, 4859-4863.

180 H. Chen, P. Li, M. Wang and L. Wang, Nickel-Catalyzed Site-Selective $\mathrm{C}-\mathrm{H}$ Bond Difluoroalkylation of 8-Aminoquinolines on the C5-Position, Org. Lett., 2016, 18, 4794-4797. 
181 C. Chen, R. Zeng, J. Zhang and Y. Zhao, RutheniumCatalyzed Difluoroalkylation of 8-Aminoquinoline Amides at the C5-Position, Eur. J. Org. Chem., 2017, 6947-6950.

182 H. Lu, D. Y. Wang and A. Zhang, Visible Light-Promoted Phosphine-Catalyzed Difluoroalkylation of Arenes and Heterocycles, J. Org. Chem., 2020, 85, 942-951.

183 S. Leilei, H. Wei, W. Jifeng, Z. Huaiyu, Z. Hua and L. Xun, Quinoxalinone as a Privileged Platform in Drug Development, Mini-Rev. Med. Chem., 2018, 18, 392-413.

184 L.-Y. Xie, Y.-L. Chen, L. Qin, Y. Wen, J.-W. Xie, J.-X. Tan, Y. Huang, Z. Cao and W.-M. He, Visible-light-promoted direct $\mathrm{C}-\mathrm{H} / \mathrm{S}-\mathrm{H}$ cross-coupling of quinoxalin-2(1H)-ones with thiols leading to 3-sulfenylated quinoxalin-2(1H)ones in air, Org. Chem. Front., 2019, 6, 3950-3955.

185 L.-Y. Xie, Y.-S. Bai, X.-Q. Xu, X. Peng, H.-S. Tang, Y. Huang, Y.-W. Lin, Z. Cao and W.-M. He, Visible-lightinduced decarboxylative acylation of quinoxalin-2(1H)ones with $\alpha$-oxo carboxylic acids under metal-, strong oxidant- and external photocatalyst-free conditions, Green Chem., 2020, 22, 1720-1725.

186 L.-Y. Xie, Y.-S. Liu, H.-R. Ding, S.-F. Gong, J.-X. Tan, J.-Y. He, Z. Cao and W.-M. He, C(sp2)-H/O-H cross-dehydrogenative coupling of quinoxalin-2(1H)-ones with alcohols under visible-light photoredox catalysis, Chin. J. Catal., 2020, 41, 1168-1173.

187 Y. W. Bao, Z. Wang, C. Chen, B. L. Zhu, Y. B. Wang, J. H. Zhao, J. Y. Gong, M. Y. Han and C. Liu, Palladium-catalyzed tandem cyclization of fluorinated imidoyl chlorides with 2-bromophenylboronic acid: Synthesis of 6-fluoroalkylphenanthridines, Tetrahedron, 2019, 75, 1450-1456.

188 C. Jin, X. H. Zhuang, B. Sun, D. Y. Li and R. Zhu, Merging Visible-Light Photoredox and Organoamine Catalysis for the C-3 Difluoroalkylation of Quinoxalin-2(1H)-Ones, Asian J. Org. Chem., 2019, 8, 1490-1494.

189 G. F. Hong, J. W. Yuan, J. H. Fu, G. Y. Pan, Z. W. Wang, L. R. Yang, Y. M. Xiao, P. Mao and X. M. Zhang, Transitionmetal-free decarboxylative C3-difluoroarylmethylation of quinoxalin-2(1H)-ones with alpha,alpha-difluoroarylacetic acids, Org. Chem. Front., 2019, 6, 1173-1182.

190 X. J. Xie, Y. F. Zhang, J. Hao and W. Wan, Ag-Catalyzed minisci C-H difluoromethylarylation of N-heteroarenes, Org. Biomol. Chem., 2020, 18, 400-404.

191 L. An, Y. L. Xiao, S. Zhang and X. G. Zhang, Bulky Diamine Ligand Promotes Cross-Coupling of Difluoroalkyl Bromides by Iron Catalysis, Angew. Chem., Int. Ed., 2018, 57, 6921-6925.

192 J. Dai, W. Lei and Q. Liu, Visible-Light-Driven Difluoroalkylation of Aromatics Catalyzed by Copper, Acta Chim. Sin., 2019, 77, 911.

193 M. J. Hossain, T. Ono, Y. Yano and Y. Hisaeda, Learning From Vitamin B-12-Mediated Reactions: Cobalt(III)Carbon-Assisted Catalytic C-H Difluoroacylation of (Hetero)Arenes through Controlled-Potential Electrolysis, ChemElectroChem, 2019, 6, 4199-4203.

194 M. Ke and Q. Song, Copper/B2pin2-catalyzed C-H difluoroacetylation-cycloamidation of anilines leading to the formation of 3,3-difluoro-2-oxindoles, Chem. Commun., 2017, 53, 2222-2225.

195 L. C. Yu, J. W. Gu, S. Zhang and X. Zhang, Visible-LightPromoted Tandem Difluoroalkylation-Amidation: Access to Difluorooxindoles from Free Anilines, J. Org. Chem., 2017, 82, 3943-3949.

196 G. Lian, J. Li, P. Liu and P. Sun, Photoredox-Catalyzed Radical Cascade Reaction To Synthesize Fluorinated Pyrrolo[1,2-d]benzodiazepine Derivatives, J. Org. Chem., 2019, 84, 9322-9329.

197 A. Z. Cao, Y. T. Xiao, Y. C. Wu, R. J. Song, Y. X. Xie and J. H. Li, Copper-catalyzed $\mathrm{C}-\mathrm{H}[3+2]$ annulation of $\mathrm{N}$-substituted anilines with alpha-carbonyl alkyl bromides via $\mathrm{C}(\mathrm{sp}(3))-\mathrm{Br} / \mathrm{C}(\mathrm{sp}(2))-\mathrm{H}$ functionalization, Org. Biomol. Chem., 2020, 18, 2170-2174.

198 Y. Gao, C. P. Li, B. Xu and H. Liu, Rapid access to difluoroalkylated pyrrolobenzodiazepines via a Pd-catalyzed C-H difluoroalkylation/cyclization cascade reaction, Org. Chem. Front., 2019, 6, 410-414.

199 H. Li, B.-J. Li and Z.-J. Shi, Challenge and progress: palladium-catalyzed sp3 C-H activation, Catal. Sci. Technol., 2011, 1, 191-206.

200 K. M. Engle, T.-S. Mei, M. Wasa and J.-Q. Yu, Weak Coordination as a Powerful Means for Developing Broadly Useful C-H Functionalization Reactions, Acc. Chem. Res., 2012, 45, 788-802.

201 D. Q. Dong, X. Gao, L. X. Li, S. H. Hao and Z. L. Wang, Sulfonylation of $\mathrm{C}(\mathrm{sp}(3))-\mathrm{H}$ bond for synthesis of 2-sulfolmethyl azaarenes catalyzed by TBAI in water, Res. Chem. Intermed., 2018, 44, 7557-7567.

202 D. Q. Dong, S. H. Hao and Z. L. Wang, Functionalization of $\mathrm{C}(\mathrm{sp}(3))-\mathrm{H}$ Bonds Adjacent to Oxygen of Alcohols under Metal-free Conditions, Mini-Rev. Org. Chem., 2017, 14, 130-135.

203 S. H. Hao, L. X. Li, D. Q. Dong and Z. L. Wang, Direct construction of sulfenylated pyrazoles catalyzed by I-2 at room temperature, Chin. J. Catal., 2017, 38, 1664-1667.

204 Y.-Z. Li, B.-J. Li, X.-Y. Lu, S. Lin and Z.-J. Shi, Cross Dehydrogenative Arylation (CDA) of a Benzylic $\mathrm{C}=\mathrm{H}$ Bond with Arenes by Iron Catalysis, Angew. Chem., Int. Ed., 2009, 48, 3817-3820.

205 X. Wan, Z. Ma, B. Li, K. Zhang, S. Cao, S. Zhang and Z. Shi, Highly Selective C-H Functionalization/Halogenation of Acetanilide, J. Am. Chem. Soc., 2006, 128, 7416-7417.

206 L. X. Li, D. Q. Dong, S. H. Hao and Z. L. Wang, Direct sulfonylation of pyrazolones with sodium sulfinates catalyzed by TBAI in water, Tetrahedron Lett., 2018, 59, 1517-1520.

207 S. Q. Yan, D. Q. Dong, C. W. Xie, W. S. Wang and Z. L. Wang, Synthesis of Bicyclic ortho-Aminocarbonitrile Derivatives Catalyzed by 1,4-Diazabicyclo 2.2.2 octane, Chin. J. Org. Chem., 2019, 39, 2560-2566.

208 D. Q. Dong, W. J. Chen, D. M. Chen, L. X. Li, G. H. Li, Z. L. Wang, Q. Deng and S. Long, Direct Synthesis of Sulfonated or Sulfenylated Pyrazolones Mediated by $\mathrm{KIO}_{3}$ and Their Anti -microbial Activity, Chin. J. Org. Chem., 2019, 39, 3190-3198. 
209 D. Q. Dong, G. H. Li, D. M. Chen, Y. Y. Sun, Q. Q. Han, Z. L. Wang, X. Xu and X. Yu, Chin. J. Org. Chem., 2020, 40, 1766-1771.

210 M. Yang, B. Su, Y. Wang, K. Chen, X. Jiang, Y.-F. Zhang, X.-S. Zhang, G. Chen, Y. Cheng, Z. Cao, Q.-Y. Guo, L. Wang and Z.-J. Shi, Silver-catalysed direct amination of unactivated $\mathrm{C}-\mathrm{H}$ bonds of functionalized molecules, Nat. Commun., 2014, 5, 4707.

211 Y. Li, J. Liu, S. Zhao, X. Du, M. Guo, W. Zhao, X. Tang and G. Wang, Copper-Catalyzed Fluoroolefination of Silyl Enol Ethers and Ketones toward the Synthesis of betaFluoroenones, Org. Lett., 2018, 20, 917-920.

212 W.-P. Li, Y.-C. Zhu, Y.-J. Zhou, H.-W. Yang and C.-J. Zhu, Visible light induced C-H monofluoroalkylation to synthesize 1,4-unsaturated compound, Tetrahedron, 2019, 75, 1647-1651.

213 J. Liu, W. Ding, Q. Q. Zhou, D. Liu, L. Q. Lu and W. J. Xiao, Enantioselective Di-/Perfluoroalkylation of beta-Ketoesters Enabled by Cooperative Photoredox/Nickel Catalysis, Org. Lett., 2018, 20, 461-464.

214 C. Li, Y. X. Cao, R. Wang, Y. N. Wang, Q. Lan and X. S. Wang, Cobalt-catalyzed difluoroalkylation of tertiary aryl ketones for facile synthesis of quaternary alkyl difluorides, Nat. Commun., 2018, 9, 4951.

215 C. Li, Y. X. Cao, R. X. Jin, K. J. Bian, Z. Y. Qin, Q. Lan and X. S. Wang, Highly stereoselective nickel-catalyzed difluoroalkylation of aryl ketones to tetrasubstituted monofluoroalkenes and quaternary alkyl difluorides, Chem. Sci., 2019, 10, 9285-9291.

216 W. Yu, Y. Ouyang, X. H. Xu and F. L. Qing, Visible LightInduced Methoxycarbonyldifluoromethylation of Trimethylsilyl Enol Ethers and Allyltrimethylsilanes with $\mathrm{FSO}_{2} \mathrm{CF}_{2} \mathrm{CO}_{2} \mathrm{Me}$, Chin. J. Chem., 2018, 36, 1024-1030.

217 H. Jiang, Y. Cheng, R. Wang, M. Zheng, Y. Zhang and S. Yu, Synthesis of 6-alkylated phenanthridine derivatives using photoredox neutral somophilic isocyanide insertion, Angew. Chem., Int. Ed., 2013, 52, 13289-13292.

218 Y. Yuan, W. Dong, X. Gao, X. Xie and Z. Zhang, Sodium Sulfite-Involved Photocatalytic Radical Cascade Cyclization of 2-Isocyanoaryl Thioethers: Access to 2- $\mathrm{CF}_{2} / \mathrm{CF}_{3}-$ Containing Benzothiazoles, Org. Lett., 2019, 21, 469-472.

219 F. Ding, Y. Jiang, K. Lin and L. Shi, Tandem radical cyclization for the construction of 1-difluoroalkylated isoquinolines via $\mathrm{Cu}$ catalyzed and visible light-promoted pathways, Org. Biomol. Chem., 2018, 16, 1812-1815.

220 X. X. Ma, S. Y. Mai, Y. Zhou, G. J. Cheng and Q. L. Song, Dual role of ethyl bromodifluoroacetate in the formation of fluorine-containing heteroaromatic compounds, Chem. Commun., 2018, 54, 8960-8963.

221 X. X. Yu, Y. Zhou, X. X. Ma and Q. L. Song, Transition metal-free assembly of 1,3,5-triazines using ethyl bromodifluoroacetate as C1 source, Chem. Commun., 2019, 55, 8079-8082.

222 A. Prieto, R. Melot, D. Bouyssi and N. Monteiro, C-H Difluoroalkylation of Aldehyde Hydrazones with
Functionalized Difluoromethyl Bromides under Copper Catalysis, ACS Catal., 2016, 6, 1093-1096.

223 A. Prieto, R. Melot, D. Bouyssi and N. Monteiro, Palladium-Catalyzed C(sp2)-H Alkylation of AldehydeDerived Hydrazones with Functionalized Difluoromethyl Bromides, Angew. Chem., Int. Ed., 2016, 55, 1885-1889.

224 P. Xu, G. Wang, Y. Zhu, W. Li, Y. Cheng, S. Li and C. Zhu, Visible-Light Photoredox-Catalyzed C-H Difluoroalkylation of Hydrazones through an Aminyl Radical/Polar Mechanism, Angew. Chem., Int. Ed., 2016, 55, 2939-2943.

225 J. Xie, T. Zhang, F. Chen, N. Mehrkens, F. Rominger, M. Rudolph and A. S. Hashmi, Gold-Catalyzed Highly Selective Photoredox $\mathrm{C}(\mathrm{sp}(2))-\mathrm{H}$ Difluoroalkylation and Perfluoroalkylation of Hydrazones, Angew. Chem., Int. Ed., 2016, 55, 2934-2938.

$226 \mathrm{M}$. Ke and Q. Song, Copper-Catalyzed C(sp(2))-H Difluoroalkylation of Aldehyde Derived Hydrazones with Diboron as Reductant, J. Org. Chem., 2016, 81, 3654-3664.

227 J. X. Li, L. Li, M. D. Zhou and H. Wang, Visible-light-promoted organic-dye-catalyzed three-component coupling of aldehydes, hydrazines and bromodifluorinated reagents, Org. Chem. Front., 2018, 5, 1003-1007.

228 P. J. Xia, Z. P. Ye, D. Song, J. W. Ren, H. W. Wu, J. A. Xiao, H. Y. Xiang, X. Q. Chen and H. Yang, Photocatalytic reductive radical-radical coupling of $\mathrm{N}, \mathrm{N}^{*}$-cyclicazomethine imines with difluorobromo derivatives, Chem. Commun., 2019, 55, 2712-2715.

229 Y. Zi, M. Lange and I. Vilotijevic, Enantioselective Lewis base catalyzed phosphonyldifluoromethylation of allylic fluorides using a C-silyl latent pronucleophile, Chem. Commun., 2020, 56, 5689-5692.

230 G. K. S. Prakash and J. Hu, Selective Fluoroalkylations with Fluorinated Sulfones, Sulfoxides, and Sulfides, Acc. Chem. Res., 2007, 40, 921-930.

231 C. Ni, M. Hu and J. Hu, Good Partnership between Sulfur and Fluorine: Sulfur-Based Fluorination and Fluoroalkylation Reagents for Organic Synthesis, Chem. Rev., 2015, 115, 765-825.

$232 \mathrm{~J}$. Hu, Nucleophilic, radical, and electrophilic (phenylsulfonyl)difluoromethylations, J. Fluorine Chem., 2009, 130, 1130-1139.

233 T. Chatterjee, N. Iqbal, Y. You and E. J. Cho, Controlled Fluoroalkylation Reactions by Visible-Light Photoredox Catalysis, Acc. Chem. Res., 2016, 49, 2284-2294.

234 M. Pohmakotr, D. Soorukram, C. Kuhakarn and V. Reutrakul, Synthesis of gem-Difluoromethylenated Compounds Employing $\quad \alpha, \alpha$-Difluoro- $\alpha$-phenylsulfanyl$\alpha$-trimethylsilylmethane $\left(\mathrm{PhSCF}_{2} \mathrm{SiMe}_{3}\right)$ as a gemDifluoromethylene Building Block, Synlett, 2014, 25582573.

235 Q. Xie, Z. Zhu, C. Ni and J. Hu, Nucleophilic (Phenylsulfonyl/arylthio)difluoromethylation of Aldehydes with $\mathrm{TMSCF}_{2} \mathrm{Br}$ : A Three-Component Strategy, Org. Lett., 2019, 21, 9138-9141. 